\title{
Predicting and auralizing acoustics in classrooms
}

\section{Christensen, Claus Lynge}

\section{Published in:}

Acoustical Society of America. Journal

Publication date:

2005

\section{Document Version}

Publisher's PDF, also known as Version of record

Link back to DTU Orbit

Citation (APA):

Christensen, C. L. (2005). Predicting and auralizing acoustics in classrooms. Acoustical Society of America. Journal, 117(4), 2465-2465.

\section{General rights}

Copyright and moral rights for the publications made accessible in the public portal are retained by the authors and/or other copyright owners and it is a condition of accessing publications that users recognise and abide by the legal requirements associated with these rights.

- Users may download and print one copy of any publication from the public portal for the purpose of private study or research.

- You may not further distribute the material or use it for any profit-making activity or commercial gain

- You may freely distribute the URL identifying the publication in the public portal

If you believe that this document breaches copyright please contact us providing details, and we will remove access to the work immediately and investigate your claim. 


\title{
Session 3aAA
}

\section{Architectural Acoustics, Speech Communication and Psychological and Physiological Acoustics: Possibilities and Problems with Auralizations of Classroom Acoustics}

\author{
Robert C. Coffeen, Chair \\ Univ. of Kansas, School of Architectural and Urban Design, Lawrence, KS 66045
}

Chair's Introduction-8:30

Invited Papers

$8: 35$

3aAA1. Predicting and auralizing acoustics in classrooms. Claus Lynge Christensen (Odeon A/S c/o Acoust. Technol., Oersted DTU, Bldg. 352, DK-2800 Lyngby, Denmark)

Although classrooms have fairly simple geometries, this type of room is known to cause problems when trying to predict their acoustics using room acoustics computer modeling. Some typical features from a room acoustics point of view are: Parallel walls, low ceilings (the rooms are flat), uneven distribution of absorption, and most of the floor being covered with furniture which at long distances act as scattering elements, and at short distance provide strong specular components. The importance of diffraction and scattering is illustrated in numbers and by means of auralization, using ODEON 8 Beta.

\section{$8: 55$}

3aAA2. Subjective evaluation of auralizations created from multi-channel anechoic recordings of a talker in motion. Michelle C. Vigeant and Lily M. Wang (Architectural Engr. Prog., Univ. of Nebraska-Lincoln, Peter Kiewit Inst., 1110 S. 67th St., Omaha, NE 68182-0681, mvigeant@unlnotes.unl.edu)

A high degree of speech intelligibility is very important in educational environments. When designing such spaces, like classrooms, auralizations can be used to subjectively assess the degree of speech intelligibility and clarity. Auralizations are most commonly made by convolving the impulse response (IR) of an omni-directional source with a single channel anechoic speech recording. This paper explores the idea of using multi-channel recordings to create the auralizations, using a female talker in motion. An omni-directional source is split into quadrants and the IR is calculated for each section. These IR's are convolved with the appropriate channel of the anechoic recording and then the four auralizations are mixed to create one final auralization. The auralizations were made using four-channel anechoic recordings of a person walking on a platform while talking. Subjective tests were conducted to determine the ease with which subjects could identify the direction of the movement of the source in rooms with varying amounts of absorption. This method can be used to create more realistic classroom auralizations, as teachers typically move around the room as they teach. [Work supported by the National Science Foundation.]

\section{9:15}

3aAA3. ClassTalk system for predicting and auralizing speech in noise with reverberation in classrooms. Murray Hodgson and Daniel Graves (UBC Acoust. \& Noise Res. Group, SOEH, 3rd Fl., 2206 East Mall, Vancouver, BC, Canada V6T 1Z3)

This paper discusses and demonstrates the ClassTalk system for predicting, visualizing and auralizing speech in noise with reverberation in classrooms. The classroom can contain a speech-reinforcement system (SRS). Male or female speech sources, SRS loudspeakers and overhead, slide or digital projectors, or ventilation-noise sources, can have four output levels. Empirical models are used to predict speech and noise levels, and Early Decay Times, from which Speech Transmission Index (STI) and Speech Intelligibility (SI) are calculated. ClassTalk visualizes the floor-plan, speech- and noise-source positions, and the receiver position. The user can walk through the room at will. In real time five quantities, background-noise level, speech level, signal-to-noise difference, STI and SI, are displayed along with occupied and unoccupied reverberation times. The sound module auralizes male or female speech mixed with the relevant noise signals, with predicted, frequency-varying reverberation superimposed using MaxxVerb. Technical issues related to the development of the sound module are discussed. The potential of the systems auralization module for demonstrating the effects of the acoustical environment and its control on speech is discussed and demonstrated.

\section{9:35}

3aAA4. Comparison of speech intelligibility scores for direct listening and headphone playback. John S. Bradley, Hiroshi Sato, Bradford N. Gover, and Natalie York (Inst. for Res. in Construction, Natl. Res. Council, Montreal Rd., Ottawa, Canada K1A 0R6)

Speech recognition test scores from direct listening and headphone playback were compared to validate the use of headphone playback for investigating the effects of varied room acoustics conditions. Varied acoustical conditions for direct listening, that were representative of classrooms, were achieved using simulated sound fields in an anechoic room with an 8-channel electro-acoustic simulation system. Eight conditions consisting of the combinations of 2 reverberation times and 4 signal-to-noise ratios were used. By recording these conditions with an acoustical mannequin, headphone playback of the same conditions could be achieved and the corresponding speech recognition scores compared. Two different approaches for correcting for the microphone and headphone 
responses were compared. Subjects also responded to a short questionnaire to describe the audible differences between direct playback and headphone listening. Because spatial characteristics were frequently found to differ between the two listening modes, further comparisons were carried out using sound fields with systematic differences in the locations of the speech and noise sources. Although there were always some audible differences between the two playback modes, it was possible to obtain the same speech recognition scores using headphone playback as with direct listening. [Work supported by CLLRnet.]

\section{9:55}

3aAA5. Auralizations and other computer model studies to predict qualitative and quantitative measures of speech intelligibility in classrooms. Gary Siebein, Hyeong-seok Kim (Univ. of Florida School of Architecture, P.O. Box 115702, Gainesville, FL 32607), and Hyun Paek (Siebein Assoc., Inc., Consultants in Architectural Acoust., Gainesville, FL 32607)

Auralizations and other computer model studies were used to predict qualitative and quantitative measures of speech intelligibility in classrooms under realistic conditions of background noise and reverberation. Speech intelligibility tests were given to college students in two classrooms and one racquetball court at 5 signal-to-noise ratios. Auralizations of the speech intelligibility tests were made from computer. Speech intelligibility tests were then administered in a sound booth using the auralized material. Fifteen different acoustical measurements related to speech intelligibility were also made at multiple locations in the actual classrooms and in the computer models of the classrooms. The scores on the speech intelligibility tests given in the actual rooms in the five noise conditions were closely duplicated in the equivalent tests conducted in a sound booth using the simulated speech signals obtained in the computer models. Both quantitative and qualitative measures of speech intelligibility in the actual rooms were accurately predicted in the computer models. Correlations (R2) between acoustical measures made in the full size classrooms and the computer models of the classrooms of 0.92 to 0.99 were found.

\section{0:15-10:30 Break}

\section{0:30}

3aAA6. Classroom auralizations using both speech and intruding noise. Robert C. Coffeen (School of Architecture and Urban Design, Marvin Hall, The Univ. of Kansas, Lawrence, KS 66045)

Continuing work with computer auralizations for education spaces—Can realistic speech auralizations be obtained for classrooms and similar spaces with disturbing ambient noise as produced by HVAC systems, noise from adjacent spaces, aircraft flyovers, and other common noise sources? Several auralizations will be presented relating to these situations.

\section{0:50}

3aAA7. Classroom sound quality-a comparison of recordings to virtual auralization. Kenneth P. Roy and Sean D. Browne (Armstrong Innovation Ctr., 2500 Columbia Ave., Lancaster, PA 17604)

Sound quality in classrooms is an important consequence of the architectural and mechanical systems design. And it is the primary factor in determining the potential for speech intelligibility, which of course is also dependent on individual listening characteristic and capabilities. So the question is "how do we assess the potential for good sound quality within the design phase of school construction?" An obvious solution is to use architectural modeling and auralization ... but the question here is "how good are these virtual techniques?" as with the use of EASE software. We will present 1st a sample of the modeling and auralization process showing the capabilities via the generated sound files. Next we will listen to recordings made in an occupied classroom both before and after architectural interventions. Then we will listen to a series of auralized classroom settings. And finally, we will compare recordings to auralizations for the same classroom. One important aspect of "real room" sound quality is occupant generated noise (non-speech), which is not an easy aspect to include in virtual models.

\section{1:10}

3aAA8. Rapid Speech Transmission Index predictions and auralizations of unusual instructional spaces at MIT's new Stata Center. David A. Conant (McKay Conant Brook Inc., 5655 Lindero Canyon Rd., Westlake Village, CA 91326)

The Stata Center for Computer, Information and Intelligence Sciences, recently opened at the Massachusetts Institute of Technology, includes a variety of oddly-shaped seminar rooms in addition to lecture spaces of somewhat more conventional form. The architects design approach prohibited following conventional, well understood room-acoustical behavior yet MIT and the design team were keenly interested in ensuring that these spaces functioned exceptionally well, acoustically. CATT-Acoustic room modeling was employed to assess RASTI through multiple design iterations for all these spaces. Presented here are computational and descriptive results achieved for these rooms which are highly-regarded by faculty. They all sound peculiarly good, given their unusual form. In addition, binaural auralizations for selected spaces are provided. 


\title{
Session 3aABa
}

\section{Animal Bioacoustics: General Topics in Hearing and Auditory Systems}

\author{
Whitlow W. L. Au, Chair \\ Hawaii Inst. of Marine Biology, P.O. Box 1106, Kailua, HI 96734
}

Chair's Introduction-7:55

Contributed Papers

8:00

3aABa1. Hearing and the round goby: Understanding the auditory system of the round goby (Neogobius melanostomus). Andrea J. Belanger and Dennis M. Higgs (Dept. of Biological Sci., Univ. of Windsor ON, 401 Sunset Ave., Windsor, ON, N9B 3P4, Canada)

The round goby (Neogobius melanostomus), is an invasive species in the Great Lakes watershed. Adult round gobies show behavioral responses to conspecific vocalizations but physiological investigations have not yet been conducted to quantify their hearing abilities. We have been examining the physiological and morphological development of the auditory system in the round goby. Various frequencies $(100 \mathrm{~Hz}$ to $800 \mathrm{~Hz}$ and conspecific sounds), at various intensities ( $120 \mathrm{~dB}$ to $170 \mathrm{~dB}$ re $1 \mathrm{~Pa}$ ) were presented to juveniles and adults and their auditory brain-stem responses (ABR) were recorded. Round gobies only respond physiologically to tones from $100-600 \mathrm{~Hz}$, with threshold varying between 145 to $155 \mathrm{~dB}$ re $1 \mathrm{~Pa}$. The response threshold to conspecific sounds was $140 \mathrm{~dB}$ re $1 \mathrm{~Pa}$. There was no significant difference in auditory threshold between sizes of fish for either tones or conspecific sounds. Saccular epithelia were stained using phalloidin and there was a trend towards an increase in both hair cell number and density with an increase in fish size. These results represent a first attempt to quantify auditory abilities in this invasive species. This is an important step in understanding their reproductive physiology, which could potentially aid in their population control. [Funded by NSERC.]

\section{$8: 15$}

3aABa2. Sound localization and auditory response capabilities in round goby (Neogobius melanostomus). Audrey K. Rollo and Dennis M. Higgs (Dept. of Biol., Univ. of Windsor, Windsor, ON, Canada N9B 3P4, audrey-rollo@ hotmail.com)

A fundamental role in vertebrate auditory systems is determining the direction of a sound source. While fish show directional responses to sound, sound localization remains in dispute. The species used in the current study, Neogobius melanostomus (round goby) uses sound in reproductive contexts, with both male and female gobies showing directed movement towards a calling male. The two-choice laboratory experiment was used (active versus quiet speaker) to analyze behavior of gobies in response to sound stimuli. When conspecific male spawning sounds were played, gobies moved in a direct path to the active speaker, suggesting true localization to sound. Of the animals that responded to conspecific sounds, $85 \%$ of the females and $66 \%$ of the males moved directly to the sound source. Auditory playback of natural and synthetic sounds showed differential behavioral specificity. Of gobies that responded, $89 \%$ were attracted to the speaker playing Padogobius martensii sounds, $87 \%$ to $100 \mathrm{~Hz}$ tone, $62 \%$ to white noise, and $56 \%$ to Gobius niger sounds. Swimming speed, as well as mean path angle to the speaker, will be presented during the presentation. Results suggest a strong localization of the round goby to a sound source, with some differential sound specificity.
$8: 30$

3aABa3. Do laboratory rearing conditions affect auditory and mechanosensory development of zebrafish (Danio rerio)? Kirsten $\mathrm{R}$. Poling, Eva Jaworski, Kristen R. Fantetti, and Dennis M. Higgs (Dept. of Biol., Univ. of Windsor, Windsor, ON, Canada N9B 3P4, kpoling@uwindsor.ca)

The effect of anthropogenic noise on the fish auditory system has become of increasing concern due to possible detrimental effects of intense sounds on auditory function and structures. This is especially problematic when raising fish in laboratory and aquaculture settings using filtration and aeration, which increase sound levels. To assess the effects of laboratory rearing conditions, one group of zebrafish (Danio rerio) embryos ("controls") were placed into aerated aquaria in a normal laboratory rearing environment. A second set of embryos ("quiet") were reared in aquaria with no aeration or filtration in a sound-resistant room. The intensity difference between the two sets of tanks was over $30 \mathrm{~dB}$. Preliminary data show that there was no affect of differential rearing environments on saccular hair cell numbers or on hearing ability in fish up to $25 \mathrm{~mm}$ total length. However, rearing environment did affect neuromast number. "Quiet" fish had higher numbers of both cephalic and trunk superficial neuromasts, relative to controls. This difference was maintained up to 11 $\mathrm{mm}$ total length (the size at which canal formation begins). This suggests that acoustic environments normally found in the laboratory do not affect development of hearing in zebrafish, although laboratory acoustics may affect mechanosensory development.

\section{$8: 45$}

3aABa4. The effect of sweep direction on avian auditory brainstem responses. Elizabeth Brittan-Powell, Amanda Lauer, Julia Callahan, Robert Dooling (Dept of Psych., Univ. of Maryland, College Park, MD 20742), Marjorie Leek (Army Medical Ctr., Washington, DC 20307), and Otto Gleich (Univ. of Regensburg, 93042 Regensburg, Germany)

In mammals, brief rising frequency sweeps result in increased amplitudes for both auditory brainstem response (ABR) and compound action potential (CAP) recordings (Dau, 2000; Shore and Nuttall, 1985). The rising sweep is thought to result in increased synchronous activity. Changing the direction of the sweep exaggerated the delay of processing along the basilar membrane and decreased synchrony of neural responses. Here we recorded ABRs from budgerigars, canaries, and zebra finches to a variety of stimulus parameters, including rising and falling sweeps with different sweep rates, determined by changing duration and frequency range. Both linear and nonlinear sweeps in frequency over time were tested. Results show that rising sweeps produce larger peak amplitudes, shorter latencies and changes in wave morphology such as a narrower wave 1 width than falling sweeps, suggesting greater synchrony of response to sweeps moving from low to high frequency. These data are consistent with mammalian results, but with a different time scale related to temporal characteristics of cochlear stimulation on the short basilar papilla in birds. [Work supported by NIH DC00198, DC001372, DC04664.] 
3aABa5. Novel technique for rapid screening of tinnitus in rats. Jeremy G. Turner, Thomas J. Brozoski, Jennifer L. Parrish, Carol A. Bauer, Larry F. Hughes, and Donald M. Caspary (Southern Illinois Univ. School of Medicine, P.O. Box 19620, Springfield, IL 62794-9620, jturner@siumed.edu)

Measuring tinnitus in laboratory animals is difficult, involving weeks or months of operant training. Preliminary data suggest that rapid screening for tinnitus in rats can be accomplished using an unconditioned acoustic startle reflex. In control animals, a gap in an otherwise constant acoustic background inhibits a subsequent startle response to a sound impulse. If, however, the background signal is qualitatively similar to the animal's tinnitus, poorer detection of the gap and less inhibition of the startle might be expected. Fourteen animals with putative tinnitus at $10 \mathrm{kHz}$ and 13 control animals were tested for gap detection using three different background signals: broadband noise, and filtered bandpass noise centered either at $16 \mathrm{kHz}(15.5-16.5 \mathrm{kHz})$ or at their suspected tinnitus locus of 10 $\mathrm{kHz}(9.5-10.5 \mathrm{kHz})$. As predicted, animals with evidence of tinnitus exhibited significantly worse gap detection at $10 \mathrm{kHz}$, and were not significantly different than control animals at $16 \mathrm{kHz}$ and broadband noise. These results suggest a new methodology for rapidly detecting tinnitus in individual animals. Equipment donated by Hamilton-Kinder Inc Behavioral Testing Systems in the memory of SIU graduate Dorothy Jean Kinder (Walker). [Work supported by NIH grants AG023910-01 (JT), DC4830 (TB \& CB), and DC00151 (DC).]

\section{9:15}

3aABa6. Emotional learning modulates the rats sensitivity to transient changes in correlation between sounds. Juan Huang, Liang Li, Zhigang Yang, Junli Ping (Dept. of Psych., Natl. Key Lab. on Machine Percept. Speech and Hearing Res. Ctr., Peking Univ., Beijing 100871, China), Xian Liu, Yixin Chen, and Xihong Wu (Peking Univ., Beijing 100871, China)

Humans are sensitive to small discrepancies between two sounds, and can detect a transient change in sound correlation. Here, prepulse inhibition of the startle reflex was used to examine rats sensitivity to transient changes in correlation between two correlated broadband noises, which were delivered by two spatially separated loudspeakers. The results show that either an uncorrelated noise fragment (UCNF, a drop of inter-sound correlation from 1.00 to 0 and then return to 1.00) or an anti-phase noise fragment (APNF, a drop of inter-sound correlation from 1.00 to -1.00 and then return to 1.00) could be detected by rats, since each of the changes in correlation could act as a prepulse stimulus to inhibit the startle reflex. The duration threshold for detecting the APNF was much lower than that for the UCNF. The detection of each of the changes in correlation was improved either by prolonged testing or by temporally pairing the UCNF or APNF with footshock. Thus similar to humans, rats also have the sensitivity to a sudden change in inter-sound correlation. Moreover, an increase of the sensitivity can be induced in rats either by repeated exposure to the change in correlation or by emotional learning.
3aABa7. The role of tragus on echolocating bat, Eptesicus fuscus. Chen Chiu (Neurosci. and Cognit. Sci. Program, Univ. of Maryland, College Park, MD 20742) and Cynthia Moss (Univ. of Maryland, College Park, MD 20742)

Echolocating bats produce ultrasonic vocal signals and utilize the returning echoes to detect, localize and track prey, and also to avoid obstacles. The pinna and tragus, two major components of the bats external ears, play important roles in filtering returning echoes. The tragus is generally believed to play a role in vertical sound localization. The purpose of this study is to further examine how manipulation of the tragus affects a free-flying bat's prey capture and obstacle avoidance behavior. The first part of this study involved a prey capture experiment, and the bat was trained to catch the tethered mealworms in a large room. The second experiment involved obstacle avoidance, and the bat's task was to fly through the largest opening from a horizontal wire array without touching the wires. In both experiments, the bat performed the tasks under three different conditions: with intact tragus, tragus-deflection and recovery from tragus-deflection. Significantly lower performance was observed in both experiments when tragi were glued down. However, the bat adjusted quickly and returned to baseline performance a few days after the manipulation. The results suggest that tragus-deflection does have effects on both the prey capture and obstacle avoidance behavior. [Work supported by NSF.]

\section{9:45}

3aABa8. Minimum audible angles for aerial pure tones in a northern elephant seal (Mirounga angustirostris). Marla M. Holt, Ronald J. Schusterman (UC Santa Cruz Long Marine Lab., 100 Shaffer Rd, Santa Cruz, CA 95060), Brandon L. Southall (UCSC Long Marine Lab. and NOAA Fisheries Acoust. Program, Silver Spring, MD 20910), and David Kastak (UC Santa Cruz Long Marine Lab., Santa Cruz, CA 95060)

Recent work has shown that several pinniped species localize aerial broadband signals as accurately as some terrestrial carnivores. Additionally, both harbor seals and California sea lions can better localize both the lower and higher frequencies of their hearing range compared to performance at intermediate frequencies. These results are congruent with the duplex theory of sound localization which states that low frequencies are localized by interaural time differences while high frequencies are localized by interaural intensity differences. Northern elephant seals are land breeding pinnipeds whose range of best hearing sensitivity is shifted toward lower frequencies compared to other pinnipeds tested thus far. In this study, we tested a female northern elephant seal in a hemi-anechoic chamber at six frequencies ranging between 0.8 and $16 \mathrm{kHz}$ that were presented at levels approximately $25 \mathrm{~dB}$ above threshold. A left/right behavioral procedure was used to measure minimum audible angles (MAAs) at 75 percent correct discrimination. MAAs ranged from approximately three to fifteen degrees. Best performance occurred at the lower frequencies while worse performance occurred at the two highest test frequencies. Unlike sea lions and harbor seals, this subject showed a decreased ability to utilize interaural intensity differences above $4 \mathrm{kHz}$. 


\title{
Session 3aABb
}

\section{Animal Bioacoustics: Animal Acoustic Repertoires: Characterization and Classification}

\author{
Volker Deecke, Chair \\ Univ. of British Columbia, Marine Mammal Research Unit, 6248 BioSciences Rd., Vancouver, BC V6T 1Z4, Canada
}

Contributed Papers

\section{0:15}

3aABb1. Variation in acoustic activity of North Atlantic right whales in three critical habitat areas in 2004. Susan E. Parks and Christopher W. Clark (Bioacoustics Res. Program, Cornell Univ., 159 Sapsucker Woods Rd., Ithaca, NY 14850)

The North Atlantic right whale is a critically endangered species. Five major habitat areas have been defined based on the presence of groups of right whales during different seasons of the year. Autonomous seafloor acoustic recorders, "pop-ups," have been used over the past five years to identify the presence/absence of right whales in Cape Cod Bay, Massachusetts by detection of right whale contact calls. These recordings have shown the effectiveness of using acoustics to monitor right whale behavior. In 2004, multi-unit arrays of pop-ups were deployed in three critical habitat areas; Cape Cod Bay (January-April), Great South Channel (May), and the Bay of Fundy (August). The presence/absence of right whale calls within range of the array, estimates of the minimum number of individuals calling, the types of calls recorded, and the diurnal patterns of sound production were measured to compare the acoustic repertoire use of right whales in these three habitats. These data were compared to visual survey data in the Bay of Fundy. These results can be used to compare diurnal and nocturnal behavioral patterns of the whales and provide information on behavioral differences between these three critical habitat areas.

10:30

3aABb2. Variation in acoustic behavior of delphinids in the Pacific Ocean based on school size and species composition. Shannon Rankin and Jay Barlow (SWFSC/NOAA, 8604 La Jolla Shores Dr., La Jolla, CA 92037, shannon.rankin@noaa.gov)

Variation in acoustic behavior based on school size and species composition was examined for surveys in the eastern tropical Pacific (2000), along the U.S. West Coast (2001), and in the U.S. EEZ surrounding Hawaii (2002). Sounds were monitored using a towed hydrophone array, and vocal schools were defined as those producing any combination of whistles, burst pulses, and/or echolocation clicks. Delphinid schools containing mixed species were consistently more vocal than single species schools. Vocal schools of Stenella attenuata, S. longirostris, Delphinus delphis, and Lissodelphis borealis were significantly larger than non-vocal schools. Vocal schools of Tursiops truncatus and Grampus griseus were somewhat larger than non-vocal schools, although this relationship was not significant. There was no relationship between group size and vocal activity for S. coeruleoalba, Steno bredanensis, and Globicephala spp. For species without a strong group size effect, all but $T$. truncatus were more vocal in the Hawaiian waters. The ability to use acoustic techniques in dolphin population estimation depends on their effectiveness in consistently detecting dolphin schools. This study suggests that small singlespecies schools of $S$. attenuata, S. longirostris, D. delphis, and L. borealis are more likely to be missed during acoustic monitoring in these regions.
10:45

3aABb3. Long term stability and individual distinctiveness in captive orca vocalizations. Michael Noonan and Malini Suchak (Canisius College, Buffalo, NY 14208, noonan@ canisius.edu)

With focus on the question of signature calling in killer whales, recordings from five captive orcas (of Icelandic origin) held at Marineland of Canada were compared. For the present analysis, samples of three different call syllables were selected from recordings made five years apart and from instances in which the identity of the calling whale was unambiguous due to temporary isolation, concomitant bubbling, and/or head nodding. The Raven software package was used to ascertain the frequency range, frequency $(\max )$, duration, and timing of maximum and minimum power within each sample. For two of the three call syllables, statistically significant differences were found among the five whales for call length and for the timing of maximums and minimums $(p<0.01-0.001)$. This similarly proved true for nearly all pairwise comparisons between whales, including mother-offspring dyads. By contrast, for three of four whales for which we had sufficient samples, no significant differences were found on any measure between samples taken from the same whales five years apart. These findings therefore support the notion that the voices of individual orcas are distinct from one another in ways that are stable over the course of multiple years.

\section{1:00}

3aABb4. The acoustic repertoire of bottlenose dolphins (Tursiops truncatus) from the southern Gulf of Mexico. Carmen Bazua-Duran (Lab. Acustica, Facultad de Ciencias, UNAM, Cd. Universitaria, 04510 Mexico DF, Mexico)

Bottlenose dolphins live in a variety of habitats of the world's oceans using their acoustic repertoire to communicate and inspect their environment. This work investigates the acoustic repertoire of bottlenose dolphins that inhabit a coastal lagoon of the southern Gulf of Mexico, the Laguna de Terminos and how it may relate to the dolphins' general behavioral state and herd size, and to the general characteristics of the habitat, such as visibility, depth, and sea state. Preliminary results show that bottlenose dolphins produce by far more clicks than whistles in all behavioral states (feeding, resting, social, and travel) and herd sizes, which may correlate with the decreased visibility and shallow depth of the Laguna de Terminos. Additionally, silence was found during all behavioral states, but very seldom in herds of large size. These preliminary results suggest that bottlenose dolphins are choosing when and where to produce their phonations. Therefore, more detailed studies are needed to understand how these animals are using their acoustic sense to communicate and inspect their environment. [Work supported by CONACyT-Gobierno Edo. de Campeche and PAPIIT, UNAM.] 
difficulties in applying these algorithms to vocalizations including quantifying the vocalization into a set of features and accounting for temporal variations in the vocalizations. Using generalized perceptual linear predication coefficients and a set of hidden Markov models to overcome these difficulties, beluga whale vocalizations are classified using $K$-means unsupervised classification. These classifications are then compared to labels assigned by human experts in order to determine the reliability of the classification system. The underlying goal of this research is to establish a generalized framework that can be used to analyze and classify the vocalizations of a number of species. [Work supported by the National Science Foundation under the Dr. Dolittle Project.]

\section{1:45}

3aABb7. Whale song analyses using bioinformatics sequence analysis approaches. Yian A. Chen, Jonas S. Almeida (Dept. of Biostatistics, Bioinformatics, and Epidemiology, 135 Cannon St., Medical Univ. of South Carolina, Charleston, SC 29425), and Lien-siang Chou (Inst. of Ecology \& Evolutionary Biol., NTU, Taipei, Taiwan)

Animal songs are frequently analyzed using discrete hierarchical units, such as units, themes and songs. Because animal songs and bio-sequences may be understood as analogous, bioinformatics analysis tools DNA/ protein sequence alignment and alignment-free methods are proposed to quantify the theme similarities of the songs of false killer whales recorded off northeast Taiwan. The eighteen themes with discrete units that were identified in an earlier study [Y. A. Chen, masters thesis, University of Charleston, 2001] were compared quantitatively using several distance metrics. These metrics included the scores calculated using the SmithWaterman algorithm with the repeated procedure; the standardized Euclidian distance and the angle metrics based on word frequencies. The theme classifications based on different metrics were summarized and compared in dendrograms using cluster analyses. The results agree with earlier classifications derived by human observation qualitatively. These methods further quantify the similarities among themes. These methods could be applied to the analyses of other animal songs on a larger scale. For instance, these techniques could be used to investigate song evolution and cultural transmission quantifying the dissimilarities of humpback whale songs across different seasons, years, populations, and geographic regions. [Work supported by SC Sea Grant, and Ilan County Government, Taiwan.]

WEDNESDAY MORNING, 18 MAY 2005

PLAZA A, 9:00 TO 11:15 A.M.

\title{
Session 3aAO
}

\section{Acoustical Oceanography, Underwater Acoustics and Animal Bioacoustics: Acoustics and Ocean Observatories II}

\author{
James H. Miller, Chair \\ Dept. of Ocean Engineering, Univ. of Rhode Island, Narragansett Bay Campus, Narragansett, RI 02882
}

Invited Papers

\begin{abstract}
9:00
3aAO1. Long-term variability in hydrothermal vent flow and temperature fluctuations by acoustic scintillation. Daniela Di Iorio (Dept. of Marine Sci., Univ. of Georgia, Athens, GA 30605)

An acoustical scintillation instrument is described that has been used to measure flow and temperature fluctuations at a hydrothermal vent plume. The vertical buoyancy driven flow together with the root-mean-square temperature fluctuations are obtained for vent structure $8 \mathrm{~F}$ of the Main Endeavour vent field on the Endeavour segment of the Juan de Fuca Ridge. Long term (71 days) measurements are obtained and an estimate of the heat flux density which is of the order $0.06 \mathrm{MW} \mathrm{m}^{-2}$ is calculated. Measurements also show oscillations in the log-amplitude variance that result from either plume advection or increased entrainment of ambient fluids by the ambient tidal currents, thus demonstrating the need for a long time series measurement of simultaneous horizontal and vertical
\end{abstract}


flow. Future measurements in the Main Endeavour vent field, with possible integration into the NEPTUNE seafloor observatory, will be over extended periods of time so that comparisons of these processes can be made with independent seismic data collected by NOAA/PMEL SOSUS array.

3aAO2. Real-time observations and predictions of scour burial at the Martha's Vineyard Coastal Observatory. Michael D. Richardson (Marine Geosciences Div., Naval Res. Lab., Stennis Space Center, MS 39520, mrichardson@nrlssc.navy.mil), Peter Traykovski, Arthur C. Trembanis (Woods Hole Oceanogr. Inst., Woods Hole, MA 02543), and Carl T. Friedrichs (Virginia Inst. of Marine Sci., Gloucester Point, VA 23062)

Mine burial by scour was measured in real-time using cylindrical instrumented mines and rotary and two-axis pencil beam sonar. Two mines and the sonar were connected to a shore-based facility at the Martha's Vineyard Coastal Observatory. Data on mine movement (heading, pitch, and roll), scour pit geometry, percent burial, and environmental processes responsible for scour burial including significant wave height, period, and tidal height were analyzed daily and presented on a mine burial web site. Sonar images show scour pits developing around the mines in response to storm generated significant wave heights greater than $2.0 \mathrm{~m}$. Mines pitch, change heading as they roll into scour pits. A comparison of the observed mine burial and real-time predictions using a modified version of the Whitehouse equations for wave-induced scour model exhibited good agreement suggesting mine burial by scour in similar sandy environments is predictable from bathymetry, sediment type, and measured or predicted surface wave conditions. The Coastal Observatory at Martha's Vineyard provided a unique opportunity for real-time data collection and analysis of both sediment transport events and seafloor-object interactions during storms. [Work supported by ONR.]

\section{9:40}

3aAO3. Autonomous and ship-cabled, bottom mounted sonar systems-Development, uses and issues associated with transitioning to ocean observatories. Kevin L. Williams, Russell D. Light, and Vernon W. Miller (Appl. Phys. Lab., Univ. of Washington, 1013 NE 40th St., Seattle, WA 98105, williams@apl.washington.edu)

Three bottom mounted sonar systems will be described that were built over a span of fifteen years. The complexity of deployment and sophistication of the tasks performed increased with each system. The first system is an autonomous tower with rotating sonar designed to examine backscattering from an area within $50 \mathrm{~m}$ radius of the tower. The second is a ship-cabled system that includes a diver movable tower and separate buried array for examining both backscattering and acoustic penetration into sediments. The last is a ship-cabled rail/tower system designed to carry out forward scattering and synthetic aperture backscattering measurements. All three systems are designed to remain deployed for time periods up to a couple of months. After describing the systems, their deployment and some example results, recent efforts will be described that are aimed at transitioning these types of systems to cabled ocean observatories. The overall goal of the talk is to indicate both the level of complexity that can be envisioned for bottom mounted systems as well as the new issues that must be addressed in moving to cabled ocean observatories. [Work supported by ONR.]

10:00-10:15 Break

\section{Contributed Papers}

\section{0:15}

3aA04. Development of interactive digital scientific acoustic systems for monitoring and assessment of physical and biological variables from ocean observatory platforms. Robert McClure, Tim Acker, James Dawson, and Assad E. K. Ebrahim (BioSonics, Inc., 4027 Leary Way NW, Seattle, WA 98107)

Scientific echosounders are in regular use for measurement of fish and plankton distribution and abundance, fish behavior, submersed aquatic vegetation, bottom sediment classification and water mass interface characteristics. Both fixed-aspect and mobile acoustic data are commonly collected from surface-deployed echosounders. Advancements in digital scientific echosounder technology and integration of other technologies has moved the capabilities of advanced echosounder systems into the realm of new applications and deployment possibilities. These advancements make possible the deployment of a new generation of integrated and interactive sensors which can provide high resolution, continuous, extended duration, recording, intelligent processing, and reliable monitoring and assessment of both biological and physical parameters of the ocean environment. Deployments from cabled observatories, moored and drifting buoys, manned and unmanned mobile platforms, and fully autonomous installations are all now reality. The deployment of these quality scientific instrument suites hold unlimited potential for research, resource management, and education. Existing and planned implementation of advanced digital scientific echosounder applications on observatories as well as considerations in design, deployment, and integration with other observatory and oceanic acoustic activities are discussed.

10:30

3aA05. Passive acoustic detection of fish sound production: The promise of ocean observatories. David Mann (USF College of Marine Sci., 140 7th Ave. S., St. Petersburg, FL 33701, dmann@ marine.usf.edu)

Many fishes make species-specific sounds during courtship and spawning. These sounds can be recorded and quantified to study the temporal and spatial dynamics of spawning. Fish sounds typically range in frequency from $20-1500 \mathrm{~Hz}$, and are usually inversely related to fish size. Time series of fish sound production recorded with autonomous recorders from Tampa Bay, Florida, and the mid-Atlantic bight, New Jersey illustrate how passive acoustics can be used to determine diel and seasonal timing of spawning. The great promise of ocean observatories is that they will not only provide unprecedented data on fish sound production, but that these data will be coupled with physical data on the same time and space scales. Ocean observatories will be powerful tools in studying the role of physical factors in driving variability in spawning and recruitment of fishes. 
ment. Based on both acoustic and non-acoustic performance characteristics the advantages and/or deficiencies of the FORA will be discussed for its use in future low-frequency long-range acoustic propagation research. [Work supported by ONR Ocean Acoustics Program.]

\section{1:00}

3aA07. Remote acoustic observations from a real-time observing system of the response of a shallow coastal embayment to hurricane forcing. Douglas J. Schillinger and Alex E. Hay (Dept. of Oceanogr., Dalhousie Univ., Halifax, NS, Canada B3H 4J1)

Results are presented from the acoustic remote sensing component of Dalhousie University's interdisciplinary Coastal Ocean Observatory in Lunenburg Bay, Nova Scotia, operational since June 2002 (www.cmep.ca/ bay). Remote acoustic measurements of water column velocity and backscatter are made from bottom-mounted instruments hard-wired to surface buoys. The data are telemetered in near real-time to a shore station via wireless Ethernet, and to Dalhousie over the Internet. The fixed-point measurements from the bottom pods are augmented by a vessel-mounted profiling system, and by opportunistic deployments of internally-recording instruments. Highlights from the first three years of the observing system operation will be presented, including sediment suspension from the seabed and bubble penetration from the surface during Hurricane Juan.

WEDNESDAY MORNING, 18 MAY 2005

REGENCY A, 8:00 TO 9:45 A.M.

\title{
Session 3aBBa
}

\section{Biomedical Ultrasound/Bioresponse to Vibration: Gene Therapy and Molecular Imaging}

\author{
Michalakis A. Averkiou, Chair \\ Philips Ultrasound, 22100 Bothell-Everett Hwy., Bothell, WA 98041-3003 \\ Chair's Introduction-8:00 \\ Invited Papers
}

8:05

3aBBa1. Augmented and selective delivery of liquid perfluorocarbon nanoparticles to melanoma cells with ultrasound. Michael S. Hughes, Kathryn C. Crowder, Gregory M. Lanza, and Samuel A. Wickline (322 Yalem, 216 S. Kings Hwy., Washington Univ. Med. School, St. Louis, MO 63110,msh@cvu.wustl.edu)

Previous work has demonstrated the ability of liquid perfluorocarbon (PFC) nanoparticles to deliver therapeutic agents to cells selectively by binding to specific cellular epitopes, and confirmed the ability to simultaneously image these targeted nanoparticles with ultrasound. In this study, enhanced delivery of targeted PFC nanoparticles to cells expressing the integrin avb3 using clinical levels of ultrasound energy were studied. Nanoparticles complexed with ligands targeted to avb3 were incubated with cells (C32 melanoma) that expressed avb3 in culture. Control nanoparticles were produced that carried no ligand targeted to avb3. A custom specimen holder permitted simultaneous microscopic visualization of cell interactions during exposure to calibrated levels of ultrasound energy. After nanoparticle binding to cells and application of ultrasound, a roughly 2 -fold increase in PFC content of the cells was observed. For control (nonbinding) nanoparticles, ultrasound exposure also increased PFC deposition, but the overall level was substantially less. Videodensitometric data show that nanoparticles were not destroyed by ultrasound exposure. Moreover, the alignment of nanoparticles relative to incident acoustic field demonstrate conclusively that acoustic radiation forces influence the nanoparticles and implicate these forces as participates in the enhanced delivery.

\section{$8: 25$}

3aBBa2. The application of acoustic radiation force for molecular imaging and drug delivery. Paul A. Dayton and Katherine W. Ferrara (UC Davis Biomed. Eng., 1 Shields Ave., Davis, CA 95616)

Acoustic radiation force is exerted on objects in an acoustic field. By optimizing acoustic parameters to maximize this force, ultrasound contrast agents can be manipulated and concentrated with an acoustic field. This technique can be used to enhance molecular imaging and drug delivery. Ultrasonic molecular imaging employs contrast agents such as microbubbles, nanoparticles, or liposomes coated with ligands specific for receptors expressed on cells at sites of angiogenesis, inflammation, or thrombus. Concentration of these highly echogenic contrast agents at a target site enhances the ultrasound signal received from that site, promoting 
ultrasonic detection and analysis of disease states. It is demonstrated that acoustic radiation force can be used to displace targeted contrast agents to a vessel wall, greatly increasing the number of agents binding to available surface receptors. Radiation force can also be used for microparticle-carrier based drug delivery. Acoustically active drug-carrier vehicles can be concentrated with radiation force, and then disrupted with high-intensity ultrasound bursts, resulting in local delivery of the therapeutic agent. This talk will discuss simulations and experimental results demonstrating these applications.

\section{Contributed Papers}

$8: 45$

3aBBa3. A new targeted drug delivery method using ultrasound and acoustically active lipospheres. Michaelann Shortencarier, Susannah Bloch, Paul Dayton, Kathy Ferrara (Dept. of Biomed. Eng., UC Davis, 451 East Health Sci. Dr., Davis, CA 95616), Terry Matsunaga, Rachel Labell, and Patricia Schumann (ImaRx Therapeutics, Inc., Tucson, AZ 85719)

The goal is to create a strategy for localized drug delivery using engineered delivery vehicles and ultrasound energy. These drug delivery vehicles, referred to as acoustically active lipospheres (AALs), consist of small gas bubbles surrounded by thick oil shells (where drugs can be carried) and are enclosed by an outermost lipid layer. Ultrasound radiation force can be used to displace these vehicles near the blood vessel wall, after which a higher intensity pulse can fragment the vehicle and transfer its contents to the endothelium. Blood velocity ranges from 1 to $10 \mathrm{~mm} / \mathrm{s}$ in the microvasculature. Therefore, agents in capillaries insonified by a transducer with a $1 \mathrm{~mm}$ focal beam width for 0.1 to 1 seconds can be displaced of 1 to $2 \mathrm{~mm}$. Delivery vehicles containing various fluorescent dyes within the oil layer are exposed to ultrasound in cell chambers or flowing vessels. Fluorescence intensity increases more than 10 -fold with application of radiation force and fragmentation, both over no exposure and over fragmentation pulses alone (both $P<0.001$ ). This implies radiation force is necessary to bring AALs into proximity of the cell monolayer before their destruction in order for drug delivery to occur.

\section{9:00}

3aBBa4. Delivery of anticancer drugs and antibodies into cells using ultrasound. Junru Wu, Jason Pepe (Dept. of Phys., Univ. of Vermont, Burlington, VT 05405), and Mercedes Rincon (Univ. of Vermont, Burlington, VT 05405)

It has been shown experimentally in cell suspensions that pulsed ultrasound $(2.0 \mathrm{MHz})$ could be used to deliver an anti-cancer drug (Adriamycin hydrochloride) into Jurkat lymphocytes and antibodies (goat anti rabbit $\operatorname{IgG}$ and anti mouse $\operatorname{IgD}$ ) into human peripheral blood mononuclear (PBMC) cells and Jurkat lymphocytes assisted by encapsulated microbubbles (Optison). When Adriamycin hydrochloride (ADR) was delivered, the delivery efficiency reached $4.80 \%$ and control baseline (no ultrasound and no ADR) was $0.17 \%$. When anti-rabbit $\operatorname{IgD}$ was delivered, the efficiencies were $34.90 \%$ (control baseline was $1.33 \%$ ) and $32.50 \%$ (control baseline was $1.66 \%$ ) respectively for Jurkat cells and PBMC. When goat anti rabbit IgG was delivered, the efficiencies were $78.60 \%$ (control baseline was $1.60 \%$ ) and $57.50 \%$ (control baseline was $11.30 \%$ ) respectively for Jurkat cells and PBMC.
9:15

3aBBa5. $1 \mathrm{kHz}$ vibration increases proteoglycan production in ATDC5 chondrocytes. Heather M. Argadine, Randall R. Kinnick, James F. Greenleaf (Dept. of Physio. and Biomed. Eng., Mayo Clinic College of Medicine, 200 First St. SW, Rochester, MN 55905, argadine.heather@mayo.edu), and Mark E. Bolander (Mayo Clinic College of Medicine, Rochester, MN 55905)

In vitro studies have shown that treatment with $1.5 \mathrm{MHz}$ ultrasound signal $\left(160 \mathrm{~mW} / \mathrm{cm}^{2}\right)$ at a $200 \mu$ s tone burst repeating at $1 \mathrm{kHz}$ increases proteoglycan synthesis in chondrocytes [J. Parvisi et al., J. Orthop. Res. 17, 488-494 (1999)]. It was hypothesized that a continuous $1 \mathrm{kHz}$ signal would be similar to the pulsed $1.5 \mathrm{MHz}$ signal in stimulating chondrocytes to produce proteoglycan, which may cause accelerated fracture healing. In vitro experiments were performed with ATDC5 cells, a chondrogenic clonal cell line, plated in 6-well plates for 3 to 7 days before receiving ultrasound treatments. Cells were treated with either $1.5 \mathrm{MHz}$ pulsed signal or $1 \mathrm{kHz}$ signal for 20 minutes per day for 9 to 11 days. The signals were calibrated so that the bottom of the 6-well plate moved $10 \mathrm{~nm}$ for each condition. After the final treatment, cell layers were stained with Alcian blue, which stains cartilage nodules providing a measure of chondrogenesis. Both $1.5 \mathrm{MHz}$ and $1 \mathrm{kHz}$ led to a highly significant increase in chondrogenesis compared to control. Quantitative image analysis of stained wells showed that treatments with either signal increased number of nodules 2.3 -fold $(p<0.02)$ and total area of nodules 3 -fold $(p<0.02)$ compared to controls.

\section{9:30}

3aBBa6. A theoretical study of the feasibility of acoustical tweezer: Ray acoustics approach. Jungwoo Lee and Kirk Shung (Dept. of Biomed. Eng., Univ. of Southern California, DRB132, Los Angeles, CA 90089, jungwool@usc.edu)

Optical tweezer has been found to have many biomedical applications in trapping macromolecules and cells. For the trapping mechanism, there has to be a sharp spatial change in axial optical intensity and the particle size must be much greater than the wavelength. Similar phenomenon may exist in acoustics. This work was undertaken to demonstrate theoretically that it is possible to acoustically trap particles near the focal point if certain conditions are met. Acoustic force exerted on fat tissue in ultrasonic fields is analyzed in ray acoustics regime where the wavelength of acoustic beam is much smaller than the size of the particle. In this paper, the analysis is therefore based on the field pattern produced by a strongly focused $100 \mathrm{MHz}$ ultrasonic transducer with Gaussian intensity distribution. The magnitude of force and Fresnel coefficients at various positions are calculated. According to the simulation results, acoustical tweezer works particularly when the beam width at focus is one wavelength and the tolerance of acoustic impedance mismatch between two media lies within 6.7\%. [Work supported by NIH Grant P41-EB2182.] 


\title{
Session 3aBBb
}

\section{Biomedical Ultrasound/Bioresponse to Vibration: Contrast Agents and Cavitation}

\author{
Paul A. Dayton, Chair \\ Univ. of California, Biomedical Engineering, 1 Shields Ave., Davis, CA 95616
}

\section{Contributed Papers}

\section{0:15}

3aBBb1. Cavitation effects of therapeutic ultrasound. Kenneth Bader and Karen Gipson (Grand Valley State Univ., Allendale, MI 49401-9403)

In order to model possible effects of cavitation on bone structure, the formation of cavitation along an acoustically reflective PVC surface was studied. The bubbles were generated by a commerical therapeutic transducer $(1 \mathrm{MHz})$, for both water and agar gel mediums. Bubble formation and activity were monitored using light scattering techniques: a $\mathrm{HeNe}$ laser was directed through the medium, and the transmitted light was detected using an optical power meter. The data show a decline in cavitation activity further from the surface and offer suggestive evidence of a boundary layer. Temporal saturation is also evident in both mediums, though an anomalous increase in transmitted light for the agar medium suggests the possibility of pressure variations due to microstreaming. Analysis of the microstreaming pattern was subsequently performed at lower frequencies in order to allow visualization with a standard laboratory microscope.

\section{0:30}

3aBBb2. Interacting bubble dynamics in Hamiltonian mechanics. Yurii A. Ilinskii, Mark F. Hamilton, and Evgenia A. Zabolotskaya (ARL, Univ. of Texas, 10000 Burnet Rd., Austin, TX 78758-4423)

A Hamiltonian formulation of the equations of motion is presented for the oscillation and 3D translation of $N$ interacting spherical bubbles. Existing alternative formulations contain second derivatives of both the bubble radius and the three coordinates of the position vector. For two or three bubbles the systems may be reduced analytically to explicit firstorder equations that can be solved with standard numerical algorithms. For large $N$, the equations cannot realistically be recast in explicit first-order form, and the leading derivatives must be evaluated by matrix inversion at each time step. The inversions introduce substantial errors for large $N$. We avoid the inversions by using Hamiltonian mechanics to derive a system of explicit first-order equations for all $N$, and which includes the influence of an applied sound field. For large $N$, the approach based on Hamiltonian mechanics results in both increased numerical accuracy and reduced computation time. The Hamiltonian system is validated by comparison with solutions obtained by alternate means. Examples are presented demonstrating various effects of bubble interaction. Although developed for modeling cavitation in shock-wave lithotripsy, the model is also applicable to bubble clouds in the ocean. [Work supported by NIH Grant EB004047 and ARL IR\&D funds.]

\section{0:45}

3aBBb3. Modeling of initial bubble growth rates during high-intensity focused ultrasound. Wayne Kreider, Michael R. Bailey, and Lawrence A. Crum (Appl. Phys. Lab, Univ. of Washington, 1013 NE 40th St., Seattle, WA 98105, wkreider@u.washington.edu)

In therapeutic applications of biomedical ultrasound, it is important to understand the behavior of cavitation bubbles. For applications that use high-intensity focused ultrasound (HIFU), both large negative acoustic pressures and heating can independently lead to bubble formation. Although neglected previously, heating during HIFU is expected to affect the growth and dissolution of bubbles by both raising the vapor pressure and promoting outgassing from gas-saturated tissues. Herein, the dynamics of a single, spherical bubble in water have been modeled using the Gilmore equation closed with an energy balance on bubble contents for calculation of pressures inside the bubble. Moreover, heat and mass transfer at the bubble wall are incorporated using the Eller-Flynn zeroth-order approximation for gas diffusion, an estimation of non-equilibrium phase change based on the kinetic theory of gases, and assumed shapes for the spatial temperature distribution in the surrounding liquid [Yasui, J. Phys. Soc. Jpn. 65, 2830-2840 (1996)]. This model allows explicit coupling of the ambient heating during HIFU to the thermodynamic state of an oscillating bubble and is currently being used to explore the growth rates of initially small, undetectable bubbles exposed to various HIFU treatment protocols. [Work supported by NIH T32-EB-001650, NIH DK43881, and NSBRI SMS00203.]

\section{1:00}

3aBBb4. Microbubble dynamics in contrast agent shell rupture. Azzdine Y. Ammi, S. Lori Bridal (CNRS UMR 7623, Université Pierre et Marie Curie, 15 rue de lécole de médecine, 75006 Paris, France, ammi@lip.bhdc.jussieu.fr), Robin O. Cleveland (Boston Univ., Boston, MA 02215), Jonathan Mamou, Grace I. Wang, and William D. O'Brien, Jr. (Univ. of Illinois, Urbana, IL 61801)

A passive cavitation detector (PCD) was used to study the features of insonified contrast agent microbubble rupture. When Optison microbubbles ruptured, the PCD signal received from single microbubbles showed the presence of post-excitation echoes identified as inertial cavitation and rebounds. Using these signals, the collapse threshold was evaluated as a function of insonation frequency, pulse duration, and pressure amplitude. For each excitation signal, 128 received time traces were recorded (200 MHz sampling, 12-bit), and frequency-domain analysis of the received signals was performed to characterize microbubble collapse. The peak rarefactional pressure threshold for detection of these emissions increased with frequency (e.g., $0.53,0.87$, and $0.99 \mathrm{MPa}$ for $0.9,2.8$, and 4.6 $\mathrm{MHz}$, respectively; 5-cycle pulse duration) and decreased with pulse duration. The modified Herring equation was used to model the bubble response at experimentally determined threshold conditions. These predicted microbubble dynamics were used to determine microbubble rupture indices based on: radial strain, peak velocity, and peak acceleration. This work provides a clearer understanding of the role of contrast agent dynamics in bubble destruction. [Work supported by the cooperative project for biomedical engineering between the University of Illinois at UrbanaChampaign, USA and the Centre National de la Recherche Scientifique, France.]

\section{1:15}

3aBBb5. The effect of size on the acoustic response of polymer-shelled ultrasound microbubble contrast agents. Susannah H. Bloch, Katherine W. Ferrara (Dept. of Biomed. Eng., Univ. of California Davis, Davis, CA 95616, kwferrara@ ucdavis.edu), and Erik R. Wisner (Univ. of California Davis, Davis, CA 95616)

Hard-shelled microbubble contrast agents differ from lipid-shelled agents in that the extra stability provided by their shell prevents dissolution of small gas bubbles: consequently, stable agents can be manufactured with diameters below $1 \mu \mathrm{m}$. Near-micron and submicron-sized contrast 
agents may enable new biomedical applications because they can traverse narrow barriers such as the lymphatic endothelium and the permeable vascular endothelium found in actively growing solid tumors. However, several studies have suggested fundamental differences in the mechanisms of acoustic response of "soft" lipid-shelled ultrasound microbubble contrast agents and "hard" albumin- or polymer-shelled agents. In particular, hard-shelled contrast agents exhibit strong echoes only after the shell is damaged and the enclosed gas is allowed to oscillate freely. Here three polymer-shelled agents with mean diameters of $0.74 \mu \mathrm{m}, 0.91 \mu \mathrm{m}$, and $1.33 \mu \mathrm{m}$ (POINT Biomedical Corp.) are investigated. Significant differences among the agents exist in the probability of response to acoustic interrogation, but not the amplitude or frequency of their acoustic response. These results are explained by a hypothesis of a pressuredependent threshold diameter above which single hard-shelled agents respond to acoustic interrogation. The implications of this hypothesis for the design of imaging methods and clinical protocols using these agents will be presented.

\section{1:30}

3aBBb6. Contrast-enhanced detection of arterial bleeding. Wenbo Luo, Vesna Zderic, Frank Starr, Marla Paun (Appl. Phys. Lab., Univ. of Washington, Box 355640, 1013 NE 40th St., Seattle, WA 98105, wenbo@u.washington.edu), and Shahram Vaezy (Univ. of Washington, Seattle, WA 98105)

The purpose of this study was to determine the feasibility and usefulness of contrast agent to detect internal bleeding. In a model of acute femoral artery injury, bleeding sites were produced percutaneously in 9 rabbits, using an $18 \mathrm{G}$ needle guided by Doppler and B-mode ultrasound. Imaging was performed pre- and post-injury and after i.v. injection of contrast agent (Optison) using $B$-mode, Color Doppler (CD), and Pulse Inversion. Gross and histological examination (light microscopy) of the injured blood vessel and the surrounding tissue were performed. Experiments with similar procedure were achieved in a pulsatile phantom with carotid arteries as targeted blood vessels in vitro. Bleeding sites could not be located in non-contrast $B$-mode sonography. After administration of contrast agent, injuries could be detected by the increased echogenicity of the extravasated blood flow. The resulted hematoma appeared as inhomogeneous hyper-echo region. Color mosaic patterns were observed in the surrounding tissue with $\mathrm{CD}$. Cluster of cavities were found within hematoma upon microscopy, which may be due to aggregated microbubbles. Bleeding sites were recognized in all contrast studies by CD; compared with only $11 \%$ in non-contrast control. Contrast-enhanced sonography is a promising tool in detection and evaluation of internal bleeding.

\section{1:45}

3aBBb7. Ultrasound contrast agents for bleeding detection and acoustic hemostasis. Vesna Zderic, Wenbo Luo, Andrew Brayman, Lawrence Crum (Appl. Phys. Lab. and Bioengineering, Univ. of Washington, 1013 NE 40th St., Seattle, WA 98105, vesna@ u.washington.edu), and Shahram Vaezy (Univ. of Washington, Seattle, WA 98105)

Objective: To investigate the application of ultrasound contrast agents (UCA) in improving both therapeutic and diagnostic aspects of ultrasound-guided High Intensity Focused Ultrasound (HIFU) therapy. Methods: Incisions ( $3 \mathrm{~cm}$ long, $0.5 \mathrm{~cm}$ deep) were made in rabbit livers (in anterior surface for HIFU treatment, or posterior surface for bleeding detection). UCA Optison $(\sim 0.1 \mathrm{ml} / \mathrm{kg})$ was injected into mesenteric vein or ear vein. A HIFU applicator $\left(5.5 \mathrm{MHz}, 6400 \mathrm{~W} / \mathrm{cm}^{2}\right)$ was scanned manually over the incision until hemostasis was achieved. Occult bleeding was monitored with Doppler ultrasound. Results: The presence of Optison produced $37 \%$ reduction in hemostasis times normalized to initial bleeding rates. Gross and histological observations showed similar appearance of HIFU lesions produced in the presence of Optison and control HIFU lesions. The temperature reached $100^{\circ} \mathrm{C}$ in both HIFU only and HIFU +UCA treatments. Tension strength of hemostatic liver incisions was $0.9 \pm 0.5 \mathrm{~N}$. Almost no bleeding could be detected before Optison injection. First appearance of contrast enhancement localized at the bleeding site was $15 \mathrm{~s}$ after Optison injection, and lasted for $\sim 50 \mathrm{~s}$. Conclusion: The presence of UCA during HIFU treatment of liver incisions resulted in shortening of HIFU application times and better visualization of bleeding sites.

\title{
Session 3aMU
}

\section{Musical Acoustics: General Topics in Musical Acoustics (Lecture/Poster Session)}

\author{
Gary P. Scavone, Cochair \\ McGill Univ., Music Technology, Faculty of Music, Montreal, QC H3A, 1E3, Canada \\ R. Dean Ayers, Cochair \\ Southern Oregon Univ., 1250 Siskiyou Blvd., Ashland, OR 97520
}

Contributed Papers

\section{8:00}

3aMU1. Phase preservation in musical signals. V. Vijayakumar and C. Eswaran (Multimedia Univ., Jalan Air Keroh Lama, 75450 Melaka, Malaysia)

The intensity variations of the harmonics of musical instruments in the frequency domain can be interpreted to store phase information. The motivation for this arises by considering the similarity of the timbre of in- struments to that obtained through a diffraction model proposed here. The intensity modifications of an input spectrum of discrete frequencies of unit intensity into the known spectra of different musical instruments have been found to match that of known instruments. According to diffraction theory, the modifying envelope encodes phase information. By considering the similarity, it is proposed that musical instrument timbre store phase information. It is suggested that timbre itself could have diffraction origins. Specific examples of musical instruments are considered to illustrate this intepretation. 
8:15

3aMU2. Practical system for recording spatially lifelike 5.1 surround sound and 3D fully periphonic reproduction. Robert E. (Robin) Miller III (FilmakerTechnol., 606 W. Broad St., Bethlehem, PA 18018)

In acoustic spaces that are played as extensions of musical instruments, tonality is a major contributor to the experience of reality. Tonality is described as a process of integration in our consciousness over the reverberation time of the room of many sonic arrivals in three dimensions, each directionally coded in a learned response by the listeners unique head-related transfer function (HRTF). Preserving this complex 3D directionality is key to lifelike reproduction of a recording. Conventional techniques such as stereo or 5.1-channel surround sound position the listener at the apex of a triangle or the center of a circle, not the center of the sphere of lifelike hearing. A periphonic reproduction system for music and movie entertainment, Virtual Reality, and Training Simulation termed PerAmbio $3 \mathrm{D} / 2 \mathrm{D}$ (Pat. pending) is described in theory and subjective tests that capture the 3D sound field with a microphone array and transform the periphonic signals into ordinary 6-channel media for either decoderless 2D replay on 5.1 systems, or lossless 3D replay with decoder and five additional speakers. PerAmbio 3D/2D is described as a practical approach to preserving the spatial perception of reality, where the listening room and speakers disappear, leaving the acoustical impression of the original venue.

\section{$8: 30$}

3aMU3. The glissando illusion: A spatial illusory contour in hearing. Diana Deutsch, Kamil Hamaoui (Dept. of Psych., Univ. of California, San Diego, La Jolla, CA 92093, ddeutsch@ucsd.edu), and Trevor Henthorn (Univ. of California, San Diego, La Jolla, CA 92093)

In the glissando illusion (originally demonstrated by Deutsch, 1995) a synthesized oboe tone of constant pitch is played together with a sine wave whose pitch repeatedly glides up and down. These are presented through two loudspeakers, placed to the listeners left and right, and are repeatedly switched between loudspeakers, such that when the oboe tone emanates from one, a segment of the sine wave emanates from the other. The oboe tone is perceived correctly as switching between loudspeakers; however the segments of the sine wave appear to be joined together seamlessly, such that a single, continuous tone is heard, which appears to emanate from a source that moves slowly around in space in accordance with its pitch motion. In the present experiment, righthanders $(n=22)$ tended strongly to hear the glissando move between left and right, and also between low and high in space, as its pitch moved between low and high. It was therefore frequently heard as tracing an elliptical path aligned diagonally between a position low and to the left when its pitch was lowest, and high and to the right when its pitch was highest. Nonrighthanders ( $n$ $=42$ ) perceived the illusion in statistically different ways.

\section{$8: 45$}

3aMU4. Effect of critical-band smoothing of musical instrument spectral data. James W. Beauchamp (School of Music and Dept. of Elec. and Computer Eng., Univ. of Illinois at Urbana-Champaign, Urbana, IL 61801, jwbeauch@uiuc.edu) and Andrew B. Horner (Hong Kong Univ. of Sci. and Technol., Clear Water Bay, Kowloon, Hong Kong)

It has been found that second-order harmonic smoothing of musical instrument spectral data can have a significant effect on timbral perception, depending on the instrument tested [McAdams et al., J. Acoust. Soc. Am. 102, 882-897 (1999)]. With critical-band smoothing, the lower harmonics, since they are in different critical bands, retain their individual amplitudes and temporal envelopes. Thus, it is hypothesized that criticalband smoothing has a lesser perceptual effect on most instrument tones than harmonic smoothing. On the other hand, upper critical bands consist of groups of harmonics. It is hypothesized that it is difficult to hear out individual harmonics within critical bands. Thus, for each band the independent harmonic temporal envelopes can be replaced by a composite rms-amplitude envelope. Spectra within bands can be replaced by timeaveraged spectra. Alternatively, time-dependent amplitude versus Bark- frequency spectral envelopes can be smoothed for each individual analysis frame. Further, amplitudes can be averaged in $\mathrm{dB}$ or linear units. Results for various processing combinations and various musical instrument sounds will be given and demonstrated.

\section{9:00}

3aMU5. An improvisation on the Middle-Eastern mijwiz; auditory roughness profiles and tension/release patterns. Pantelis N. Vassilakis (DePaul Univ., School of Music, 804 W. Belden Ave., Chicago, IL 60614-3250, pantelis@acousticslab.com)

Within western musical tradition, auditory roughness constitutes one of the principle perceptual correlates of dissonance. A previous study [P. N. Vassilakis, Selected Reports in Ethnomusicology, Vol. 12 (in press)] addressed the musical significance of auditory roughness within western as well as three non-western musical traditions. It introduced a new roughness estimation model that predicts successfully roughness ratings of synthesized complex diatonic dyads. For the present study, an application was created that uses the aforementioned model to estimate the roughness of natural signals with arbitrary spectra, as well as roughness profiles of short musical pieces. The application was used to examine if and how the pattern of tension and release within a stylized improvisation on the Middle Eastern mijwiz (double clarinet) relates to roughness changes. Tension/ release patterns were indicated by the Lebanese improviser and expert mijwiz player (Dr. A. J. Racy) and were also obtained by both musicians and non-musicians (American-raised) in a perceptual experiment. The results suggest that auditory roughness is a good predictor of the tension/ release pattern indicated by the improviser. The patterns obtained by the subjects, although not very consistent, appear to be different overall, suggesting that musical tension and release may be culture-specific concepts.

\section{9:15}

3aMU6. Visual feedback for retuning to just intonation intervals. $R$. Dean Ayers (Phys. Dept., Southern Oregon Univ., 1250 Siskiyou Blvd., Ashland, OR 97520), Peter R. Nordquist, and Justin S. Corn (Southern Oregon Univ., Ashland, OR 97520)

Musicians become used to equal temperament pitch intervals due to their widespread use in tuning pianos and other fixed-pitch instruments. For unaccompanied singing and some other performance situations, a more harmonious blending of sounds can be achieved by shifting to just intonation intervals. Lissajous figures provide immediate and striking visual feedback that emphasizes the frequency ratios and pitch intervals found among the first few members of a single harmonic series. Spirograph patterns (hypotrochoids) are also especially simple for ratios of small whole numbers, and their use for providing feedback to singers has been suggested previously [G. W. Barton, Jr., Am. J. Phys. 44(6), 593-594 (1976)]. A hybrid mixture of these methods for comparing two frequencies generates what appears to be a three dimensional Lissajous figure-a cylindrical wire mesh that rotates about its tilted vertical axis, with zero tilt yielding the familiar Lissajous figure. Sine wave inputs work best, but the sounds of flute, recorder, whistling, and a sung "oo" are good enough approximations to work well. This initial study compares the three modes of presentation in terms of the ease with which a singer can obtain a desired pattern and recognize its shape.

\section{9:30}

3aMU7. Directivity measurements of the violin during performance. Jean-François Hardy and Jean-Gabriel Migneron (Laboratoire d'acoustique de l'Université Laval, 1 Côte de la Fabrique, Québec, PQ, Canada G1K 7P4, jean-francois.hardy.1@ulaval.ca)

Many studies have shown that directivity of musical instruments is significant for the perceived sound within the hall and for performer's ease of hearing each other. The directivity patterns explain in part the differences between many common or special orchestral stage plots. Measure- 
ments of the violin's directivity have been performed using an acoustical intensimetry procedure. The precise directivity characteristics of isolated tones have been compared with the mean directivity resulting from performance of four extracts chosen among the orchestral repertoire. Results, which were measured in both horizontal and vertical planes, show interesting differences between the average directivity of each test. They lead to links with few important distinctions in the traditional violin's positions inside the orchestra.

\section{$9: 45$}

3aMU8. Application of cochlear analysis techniques to percussion in electro-acoustic music. John Mills III (ECE Dept, The Univ. of Texas, 1 Univ. Station 0803, Austin, TX 78712, nodog@mail.utexas.edu)

This presentation is focused upon the audio analysis involved in the creation of a "pseudo-score" for electro-acoustic music. This research was undertaken because the lack of an objective visual representation prevents music theorists from being able to easily discuss electro-acoustic music in written form. The "holy grail" of automatic computer music analysis has most often been the translation of a continuous pressure variation into traditional Western musical notation. Since electro-acoustic music is rarely able to be transcribed into this type of notation, the automatic analysis of electro-acoustic music confounds many previous algorithms. A top-down approach is suggested in order to extract acoustic and musical information from recordings of electro-acoustic music. Because humans are the best known system for translating a continuous pressure variation into useful acoustic information, a cochlear model, Patterson's Auditory Image Model (AIM), is used as a front end. The audio property of self-similarity of percussion has been chosen for initial study. This self-similarity property requires first that the audio property of percussion is quantified. The nuances of using AIM to determine percussion will be discussed. Models of self-similarity of percussion for varying time-scales will then be discussed.
10:00-10:15 Break

10:15

3aMU9. Fractal dimension analysis of complexity in Ligeti piano pieces. Rolf Bader (Inst. of Musicology, Univ. of Hamburg, Neue Rabenstr. 13, 20354 Hamburg, Germany)

Fractal correlation dimensional analysis has been performed with whole solo piano pieces by Gyrgy Ligeti at every 50ms interval of the pieces. The resulting curves of development of complexity represented by the fractal dimension showed up a very reasonable correlation with the perceptional density of events during these pieces. The seventh piece of Ligeti's "Musica ricercata" was used as a test case. Here, each new part of the piece was followed by an increase of the fractal dimension because of the increase of information at the part changes. The second piece "Galamb borong," number seven of the piano Etudes was used, because Ligeti wrote these Etudes after studying fractal geometry. Although the piece is not fractal in the strict mathematical sense, the overall structure of the psychoacoustic event-density as well as the detailed event development is represented by the fractal dimension plot.

\section{0:30}

3aMU10. Attack transients on lip reed instruments. Murray Campbell, Seona Bromage (School of Phys., Univ. of Edinburgh, Edinburgh EH9 3JZ, UK, d.m.campbell@ed.ac.uk), and John Chick (Univ. of Edinburgh, Edinburgh EH9 3JL, UK)

The generation of sound in a lip reed (brass) instrument relies on a non-linear coupling between two vibrating systems: the lips of the player, and the air column contained by the walls of the instrument. The nature of this coupling has been studied by filming the motion of the lips of a number of players using a specially constructed transparent mouthpiece, while simultaneously recording the pressure in the mouthpiece and at the bell of the instrument. Attention has been focused on the attack transient, and the way in which its duration and evolution depend on the extent to which the air column modes are harmonically related. For this reason the instrument chosen was the serpent; on this instrument certain fingerings give air column modes whose relationship is close to harmonic, while other fingerings give strikingly inharmonic air column modes. The relationship between measurements of transient character and judgements of the playability or responsiveness of the instrument will be discussed.

\section{0:45-10:50 Break}

All posters will be on display and authors will be at their posters from 10:50 a.m. to 12:00 noon.

3aMU11. Microrhythmic characteristics of musical instrument initial transients. Rolf Bader (Inst. of Musicology, Univ. of Hamburg, Neue Rabenstr. 13, 20354 Hamburg, Germany)

A microrhythmic analysis of initial transients of the violin, the guitar, the saxophone, the clarinet, and percussion instruments has been performed. They show characteristic patterns between instrument families, concerning the derivation of microrhythmic intervals within the tonal frequency range compared to psychoacoustic discrimination tasks by listeners. Microrhythm here is defined as the temporal distance between two amplitude peaks. As initial transients are a crucial part of the sound in terms of identification of instruments and the initial is just in the range of the second integration time of the ear, where frequency components can just be identified, these microrhythmic patterns could be a possibility for listeners to identify the instrument families. This is because microrhythmic intervals are instantaneously present and do not need an integration time span.
3aMU12. Signal-to-background-ratio preferences of normal-hearing listeners as a function of music. Jillian G. Barrett (Dept. of Communicative Sci. and Dis., California State Univ. Hayward, 25800 Carlos Bee Blvd., Hayward, CA 94542)

The primary purpose of speech is to convey a message. Many factors affect the listener's overall reception, several of which have little to do with the linguistic content itself, but rather with the delivery (e.g., prosody, intonation patterns, pragmatics, paralinguistic cues). Music, however, may convey a message either with or without linguistic content. In instances in which music has lyrics, one cannot assume verbal content will take precedence over sonic properties. Lyric emphasis over other aspects of music cannot be assumed. Singing introduces distortion of the vowel-consonant temporal ratio of speech, emphasizing vowels and de-emphasizing consonants. The phonemic production alterations of singing make it difficult for even those with normal hearing to understand the singer. This investigation was designed to identify singer-to-background-ratio (SBR) prefer- 
ences for normal hearing adult listeners (as opposed to SBR levels maximizing speech discrimination ability). Stimuli were derived from three different original songs, each produced in two different genres and sung by six different singers. Singer and genre were the two primary contributors to significant differences in SBR preferences, though results clearly indicate genre, style and singer interact in different combinations for each song, each singer, and for each subject in an unpredictable manner.

3aMU13. Normal-hearing listener preferences of music as a function of signal-to-noise-ratio. Jillian G. Barrett (Dept. of Communicative Sci. and Dis., California State Univ. Hayward, 25800 Carlos Bee Blvd., Hayward, CA 94542)

Optimal signal-to-noise ratios (SNR) for speech discrimination are well-known, well-documented phenomena. Discrimination preferences and functions have been studied for both normal-hearing and hard-ofhearing populations, and information from these studies has provided clearer indices on additional factors affecting speech discrimination ability and SNR preferences. This knowledge lends itself to improvements in hearing aids and amplification devices, telephones, television and radio transmissions, and a wide arena of recorded media such as movies and music. This investigation was designed to identify the preferred signal-tobackground ratio (SBR) of normal-hearing listeners in a musical setting. The signal was the singer's voice, and music was considered the background. Subjects listened to an unfamiliar ballad with a female singer, and rated seven different SBR treatments. When listening to melodic motifs with linguistic content, results indicated subjects preferred SBRs similar to those in conventional speech discrimination applications. However, unlike traditional speech discrimination studies, subjects did not prefer increased levels of SBR. Additionally, subjects had a much larger acceptable range of SBR in melodic motifs where the singer's voice was not intended to communicate via linguistic means, but by the pseudo-paralinguistic means of vocal timbre and harmonic arrangements. Results indicate further studies investigating perception of singing are warranted.

3aMU14. Soprano and source: A laryngographic analysis. Laura Anne Bateman (Canadian College of Performing Arts, 1701 Elgin Rd., Victoria, BC, Canada)

Popular music in the 21 st century uses a particular singing quality for female voice that is quite different from the trained classical singing quality. Classical quality has been the subject of a vast body of research, whereas research that deals with non-classical qualities is limited. In order to learn more about these issues, the author chose to do research on singing qualities using a variety of standard voice quality tests. This paper looks at voice qualities found in various different styles of singing: Classical, Belt, Legit, R\&B, Jazz, Country, and Pop. The data was elicited from a professional soprano and the voice qualities reflect industry stan- dards. The data set for this paper is limited to samples using the vowel [i]. Laryngographic (LGG) data was generated simultaneously with the audio samples. This paper will focus on the results of the LGG analysis; however, an audio analysis was also performed using Spectrogram, LPC, and FFT. Data from the LGG is used to calculate the contact quotient, speed quotient, and ascending slope. The LGG waveform is also visually assessed. The LGG analysis gives insights into the source vibration for the different singing styles.

3aMU15. Optimized interpolations and nonlinearity in numerical studies of woodwind instruments. Apostolos Skouroupathis (Dept. of Phys., Univ. of Cyprus, P.O. Box 20537, Nicosia CY-1678, Cyprus, php4as01@ucy.ac.cy)

The impedance spectra of woodwind instruments with arbitrary axisymmetric geometry are studied. Piecewise interpolations of the instruments' profile are performed, using interpolating functions amenable to analytic solutions of the Webster equation. Our algorithm optimizes on the choice of such functions, while ensuring compatibility of wave-fronts at the joining points. Employing a standard mathematical model of a singlereed mouthpiece, as well as the time-domain reflection function which is derived from our impedance results, the Schumacher equation is solved for the pressure evolution in time. Analytic checks are made to verify that, despite the nonlinearity in the reed model and in the evolution equation, solutions are unique and singularity-free.

3aMU16. An alternative solution for positioning sound sources in loudspeaker-based virtual environments. Jonas Braasch (CIRMMT, McGill Univ., Montreal, QC, Canada H3A 1E3, jb@music.mcgill.ca)

In auditory virtual environments it is often required to position an anechoic point source in three-dimensional space. When sources in such applications are to be displayed using multichannel loudspeaker reproduction systems, the processing is typically based upon simple amplitudepanning laws. This paper describes an alternative approach based on an array of virtual microphones. The aim of the system architecture design was to comply with the expectations of audio engineers and to create sound imagery similar to those associated with standard sound recording practice. In the newly designed environment, the microphones, with adjustable directivity patterns and axis orientations, can be spatially placed as desired. This way, time-delay panning can be included by simulating a non-coincident microphone array. By the use of omnidirectional microphones the panning is almost solely based on time-delay panning and thus performance can take advantage of the precedence effect. Also, the proposed architecture is flexible enough to include classic amplitude panning laws by translating the requested panning law into physically non-existing microphone directivity patterns. Moreover, the virtual directivity patterns do not have to be rotationally symmetrical. [Work supported by VRQ.] 


\title{
Session 3aNS
}

\section{Noise: Progress in Managing Occupational Noise}

\author{
Hugh Davies, Cochair \\ Univ. of British Columbia, School of Occupational and Environmental Hygiene, 2206 East Mall, \\ Vancouver, BC V6T 1Z3, Canada \\ Murray R. Hodgson, Cochair \\ Univ. of British Columbia, School of Occupational and Environmental Hygiene, 2206 East Mail, \\ Vancouver, BC V6T 1Z3, Canada
}

Chair's Introduction-8:00

Invited Papers

8:05

3aNS1. Hearing conservation practices in eight noisy industries. William E. Daniell, Susan S. Swan, Janice Camp, Martin Cohen (Dept. of Environ. and Occupational Health Sci., Univ. of Washington, Box 357234, Seattle, WA 98195), Mary M. McDaniel (Pacific Hearing Conservation, Seattle, WA 98117), John Stebbins (Dept of Labor \& Industries, Olympia, WA 98504), and Robert Leo (Dept of Labor \& Industries, Seattle, WA 98104)

This study evaluated noise exposures and hearing conservation practices at 76 companies in eight industries with high rates of workers' compensation claims for hearing loss. Nearly all companies had exposures that required a hearing conservation program, and more than half had exposures that required consideration of noise controls. The use of noise measurements and consideration of controls was low in all industries. The completeness of hearing conservation programs was strongly associated with the extent of exposure in an industry, although practices varied widely within industries. Most companies had substantial deficiencies. More than one-third did not conduct annual training, and training had shortcomings at many others. One-third had not conducted audiometry. Hearing protection was commonly underused. Reported use was highest at companies with relatively complete programs, and in industries where exposure was most prevalent and least intermittent. Many employees had difficulty estimating how often, and presumably when, their exposure was excessive. There is a need for new strategies to promote and maintain hearing conservation efforts in noisy industries. The industries with greatest margin for improvement are not the noisiest industries but those where exposure is moderate or intermittent. [Work supported by the National Institute for Occupational Safety and Health.]

\section{$8: 25$}

3aNS2. Effectiveness of the hearing conservation program: Change in hearing threshold shift incidence among industrial workers, 1978 to 2003. Hugh Davies (UBC School of Occupational and Environ. Hygiene, 2206 East Mall, Vancouver, BC, Canada V6T 1Z3, hugh.davies@ubc.ca)

Hearing conservation programs (HCP) are widely employed in preventing noise-induced hearing loss, but studies of their effectiveness have been rare. The impact of the implementation of hearing conservation programs was assessed in a large group of highly noise-exposed blue-collar workers by investigating time-trends in hearing-threshold shift incidence. Serial annual audiograms for employees of 14 British Columbia lumber mills for the period 1978 to 2003 were obtained from local regulatory-agency archives. Audiograms and concomitant otological medical histories were linked to subjects' work histories and noise exposure data. Multivariable Cox proportional hazard models were used to model the incidence of hearing threshold shift while controlling for age, baseline level of hearing loss, and other potential confounders. A total of 109257 audiograms were associated with 10590 subjects. Mean noise exposure in this group was $91.4 \mathrm{dBA}(\mathrm{A})$. Mean interval between hearing tests was 566 days and mean age at first threshold shift was 44. Forty-six percent of subjects had at least one OSHA significant threshold shift during follow up. Preliminary analyses indicated a trend toward lower incidence of threshold shifts over the study period, with incidence in 5 approximately equal 5-year periods from 1978 to 2003 being $3.2 \%, 6.6 \%, 4.9 \%, 4.3 \%$ and $2.4 \%$, respectively.

\section{8:45}

3aNS3. Use and effectiveness of hearing protection in construction. Richard Neitzel and Noah Seixas (Dept. of Env. and Occup. Health Sci., Univ. of Washington, 4225 Roosevelt Way NE \#100, Seattle, WA 98105, rneitzel@u.washington.edu)

This paper presents an analysis of data on noise exposure and hearing protection device (HPD) use among construction workers drawn from several large datasets covering nine construction trades. A unique combination of one-minute dosimetry noise exposure level and simultaneous self-reported HPD use data were evaluated, as were occupational and non-occupational HPD use data collected by questionnaire as part of a longitudinal noise and hearing loss study among apprentices. Direct HPD attenuation measurements were also made on workers at their worksite. Workers reported using HPDs less than one-quarter of the time they were exposed above 85 dBA, the NIOSH Recommended Exposure Limit. Workers who reported always using HPDs in high noise were found to wear them only one-third of the time their exposures exceeded $85 \mathrm{dBA}$. Direct attenuation measurements indicated that workers achieved more 
than $50 \%$ of the rated attenuation of their HPD on average, but that the variability in achieved attenuation was large. Lastly, when the measured HPD attenuation level and use time data were combined, the effective protection afforded by HPDs was less than $3 \mathrm{~dB}$ on average, though there was substantial variation among the different trades. These results demonstrate the need for better hearing conservation programs in construction.

9:05

3aNS4. Alternative noise exposure metrics among construction workers. Noah Seixas, Rick Neitzel, Lianne Sheppard, and Bryan Goldman (DEOHS, Univ. of Washington, Box 357234, Seattle, WA 98195-7234)

There continues to be some uncertainty in the choice of noise exposure metrics for assessing the risk of hearing damage. In particular, the Leq, based on a $3 \mathrm{~dB}$ exchange rate (ER) and the Lavg, which uses a $5 \mathrm{~dB}$ ER are both in use, as are various metrics representing exposure peaks. This paper presents an analysis of a large database of noise exposures among construction workers, comparing several noise metrics, and their application to a cohort of construction workers, followed audiometrically over a three year period. Metrics examined were the Lavg, Leq, and Lmax, expressing average levels. Two novel metrics were derived from these monitored metrics, Leq/Lavg, and Lmax/Leq, as measures of exposure variability and peakiness. A total of 730 workshifts, including data on 361492 min of exposure to workers in 9 trades were examined. Correlations between average metrics (Leq, Lavg, Lmax) are generally very high, while the variability metrics are poorly correlated with average levels and with each other, indicating that they characterize different aspects of exposure. The task-specific estimates of individual exposure, using the various metrics, were applied to the cohorts work history and their effects on changes in hearing were assessed.

\section{9:25}

3aNS5. Special issues in hearing loss prevention in the Canadian military environment. Christian Giguère and Chantal Laroche (Univ. of Ottawa, 451 Smyth Rd., Ottawa, ON, Canada K1H 8M5)

Noise can be particularly noxious to hearing in the military. The personnel regularly face a wide range of noise-hazardous situations, many of which are seldom encountered in other work environments. High noise levels are associated with the operation of small arms and large caliber weapons, combat vehicles, aircrafts, ships and vessels, and industrial equipment. This can induce permanent and temporary hearing loss, compromise speech communication, interfere with the detection and localization of sound sources and warning sounds and thus, can jeopardize life or safety of the personnel. This paper will review the essential elements of a hearing loss prevention program proposed for the Canadian Armed Forces. The ultimate goal is to preserve hearing health as well as all hearing abilities necessary for effective operations. The program has been designed to meet the noise measurement and hazard investigation procedures, limits on noise exposure, use of hearing protection and other regulatory measures contained in the Canadian Occupational Health and Safety (COHS) Regulations (Part VII: Levels of Sound), while addressing the particular nature of the military environment. The paper will focus on issues that are not typically found in other occupational environments (variable work schedules, excessive impulse noise, extended exposures, communication devices).

\section{9:45}

3aNS6. In-situ personal assessment of hearing protector performance-recommendations for an updated standard. Jérémie Voix, Frédéric Laville (École de technologie supérieure, Université du Québec, Montreal (PQ), Canada H3C 1K3), and Jean Zeidan (Sonomax Hearing Healthcare Inc., Montreal (PQ), Canada H4P 2E2)

Standardized method currently used to assess Hearing Protection Devices (HPD) performance have little correspondence with protection achieved by users in practice. To overcome this problem new measurement method are being proposed. They no longer rely on statistically-based, population-based estimate, such as the Noise Reduction Rating (NRR), but on an individual assessment of the performance of the HPD. These new methods are highly beneficial for effective Hearing Conservation Programs as they provide individual attenuation values for noise-exposed workers in lieu of the statistically-based laboratory performance estimate, but their implementation will necessitate complete revision of existing standards. Among the issues to be addressed, are the aspect of the accuracy of the measurements performed by those methods and associated devices and also the replacement of any single number rating of HPD (such as the NRR) in existing standards. Solutions to address theses issues and some general guidelines for an updated standard will be presented.

\section{0:05-10:20 Break}

\section{0:20}

3aNS7. Active hearing protectors: Prospects for improving speech intelligibility while maintaining noise reduction and audibility of warning alarms. Anthony J. Brammer, Donald R. Peterson, Martin G. Cherniack, and Subhash Gullapalli (Ergonomic Technol. Ctr., Univ. of Connecticut Health Ctr., 263 Farmington Ave., Farmington, CT 06030, anthonybrammer@ hotmail.com)

Users of hearing protection devices (HPDs), including the communication headsets and helmets commonly worn by aircraft air and ground crews, emergency responders, military personnel, and persons in industry working in hostile environments, have long complained that HPDs interfere with their ability to communicate and to hear warning alarms. There thus appears to be a need for additional specialized HPDs designed specifically for these situations. The technology of active noise control together with signal processing applied to the communication channel of the HPD may provide a potential solution. The combination could improve noise reduction at low frequencies and enhance the intelligibility of speech reproduced by the communication channel, while maintaining the overall noise reduction within occupational exposure requirements, and assisting the perception of external warning alarms. A 
strategy for achieving these goals using an active circumaural earmuff equipped with sub-band processing will be described, in which parallel controllers optimize the speech signal-to-noise ratio in bands that contribute most to intelligibility. The perception of a tonal alarm is assisted by binaural feed through to the earphones, within the constraints imposed by occupational exposure limits, and by sub-band processing to reduce the upward spread of masking.

\title{
Contributed Papers
}

10:40

3aNS8. The combined effect of noise and carbon monoxide on hearing thresholds of exposed workers. Adriana Lacerda, Tony Leroux, and Jean-Pierre Gagn (Univ. of Montreal, C.P.2168, succursale centre-ville, H3C 3J7, Montreal, Canada, Adriana.lacerda@umontreal.ca)

Animal models have been used to demonstrate the potentiation of noise-induced hearing loss (NIHL) by carbon monoxide. It has been shown that the addition of carbon monoxide to otherwise safe noise exposure levels produces significant NIHL in rats. However, the effects of chronic exposure to low level of carbon monoxide in a noisy work environment are still unknown. The aim of this study was to compare the hearing thresholds of a group of workers exposed to noise and carbon monoxide (Group 1) to another group of workers where carbon monoxide exposure is absent or negligible (Group 2). The analysis was based on 9396 audiograms collected by the Quebec National Public Health Institute between 1983 and 1996. The results show significantly poorer hearing thresholds at high frequencies $(3,4$, and $6 \mathrm{kHz})$ for the carbon monoxide exposed group $(p<0.001)$. The potentiation effect also varied according to years of exposure in work place; a larger effect is observed for workers with between 15 to 20 years of exposure $(p<0.001)$. This study provides the first demonstration of a potentiation effect of NIHL by carbon monoxide in humans.
$10: 55$

3aNS9. Derivation of an analytic expression for the error associated with the noise reduction rating. William J. Murphy (Hearing Loss Prevention Team, NIOSH 4676 Columbia Pkwy., MS C-27, Cincinnati, $\mathrm{OH}$ 45226-1998)

Hearing protection devices are assessed using the Real Ear Attenuation at Threshold (REAT) measurement procedure for the purpose of estimating the amount of noise reduction provided when worn by a subject. The rating number provided on the protector label is a function of the mean and standard deviation of the REAT results achieved by the test subjects. If a group of subjects have a large variance, then it follows that the certainty of the rating should be correspondingly lower. No estimate of the error of a protector's rating is given by existing standards or regulations. Propagation of errors was applied to the Noise Reduction Rating to develop an analytic expression for the hearing protector rating error term. Comparison of the analytic expression for the error to the standard deviation estimated from Monte Carlo simulation of subject attenuations yielded a linear relationship across several protector types and assumptions for the variance of the attenuations.

\section{Session 3aPA}

\section{Physical Acoustics, Structural Acoustics and Vibration and Underwater Acoustics: Scattering From Non-Canonical Objects}

\author{
Steven G. Kargl, Chair \\ Applied Physics Lab., Univ. of Washington, 1013 NE 40th St., Seattle, WA 98105-6698 \\ Invited Papers
}

8:30

3aPA1. Intermediate scale measurement system for underwater acoustic measurements. Jan M. Niemiec, Paul C. Shang (NSWCCD, 9500 MacArthur Blvd., West Bethesda, MD 20817, niemiecjm@nswccd.navy.mil), and Stephan Schreppler (ONR, Code 334, Arlington, VA 22217)

The Intermediate Scale Measurement System (ISMS) provides high quality acoustic measurements on large-scale submarine models and other underwater structures in a controlled environment. Development and implementation of advanced marine technology designs require a thorough understanding of structural acoustic response mechanisms. The only practical and cost effective approach to developing this understanding is through the use of a combination of numerical models and large-scale physical models. The ISMS provides an affordable, unique capability of making precision acoustic measurements on models large enough to contain the structural detail and the instrumentation required to understand structural acoustic mechanisms and to confirm and guide the use of numerical models. This is a key element for prediction of full-scale performance and integration of advanced submarine technologies into operational systems. 
3aPA2. Scattering by truncated targets with and without boundary interactions. Philip L. Marston, Kyungmin Baik, Aubrey Espana, Curtis F. Osterhoudt (Phys. Dept., Washington State Univ., Pullman, WA 99164-2814, marston@wsu.edu), Scot F. Morse (Western Oregon Univ., Monmouth, OR 97361), Brian T. Hefner (Univ. of Washington, Seattle, WA 98105), and Florian J. Blonigen (Northeastern Univ., Boston, MA 02115)

Ray methods have been applied to the scattering of various truncated targets having wavenumber-radius products as small as 10 [F. J. Blonigen and P. L. Marston, J. Acoust. Soc. Am. 107, 689-698 (2000); S. F. Morse and P. L. Marston, ibid. 112, 1318-1326 (2002); B. T. Hefner and P. L. Marston, ARLO 2, 55-60 (2001)]. Recent work emphasizes the exploration of scattering enhancements for other situations including plastic cylinders having curved ends, truncated plastic cones, partially exposed cylinders, and objects in simulated conditions for burial in a seabed. Enhanced scattering is often associated with a locally flat outgoing wavefront. For plastic targets it has been helpful to examine the time dependence of the backscattered envelope as a function of target tilt for targets illuminated by short tone bursts. For partially exposed objects it is helpful to examine the backscattering as a function of the target exposure. For simulated buried targets, it has been helpful to excite target resonances. [Work supported by the Office of Naval Research.]

\section{9:20}

3aPA3. Finite-element modeling of acoustic scattering from realistic elastic structures. David S. Burnett (Naval Surface Warfare Ctr., Code R21, 110 Vernon Ave., Panama City, FL 32407, david.s.burnett@navy.mil)

Over the last twenty years the author has developed several finite-element codes for modeling acoustic scattering from complicated elastic structures. All the codes have employed 3-D continuum mechanics throughout the computational domain. Thin structural components, such as plates and shells, are modeled with 3-D elasticity rather than plate or shell theories. The codes are therefore fully 3-D, i.e., in both physics and geometry. The talk will first explain the rationale for this approach and touch briefly on essential aspects of previous codes. It will then focus in more detail on the current code under development, which employs relatively inexpensive commercial software well-suited for this fully 3-D approach.

\section{Contributed Papers}

9:45

3aPA4. Acoustic scattering from an obstacle in a homogeneous nonuniform moving medium via the ray method. Tatiana Semenova (Natl. Res. Council Postdoctoral Res. Associate, NASA Langley Res. Ctr., Hampton, VA 23666)

For an accurate noise prediction for aircraft in forward flight, scattering of engine noise from airframe must be included into the description of the sound field. A realistic model of acoustic scattering by airframe in flight must account for the effects of the background flow. We consider acoustic scattering from an obstacle in the presence of a time-independent background flow at low Mach number and we neglect all other effects of the variation of the medium on sound propagation. The model is based on a convected Helmholtz equation with coefficients dependent on the background flow, which can be given either analytically or by CFD. A highfrequency solution is sought by the ray acoustics with a simple method based on the Fresnel volumes of the rays employed to determine the caustic zones and the field on the caustics. The weaker effects of a tip or smooth-body diffraction are neglected and only the reflection and edge diffraction are taken into account. The source of noise is modeled as a point source. The upstream shielding effect of a boundary layer is investigated as an example and uniform versus non-uniform flow effects are illustrated. Other numerical examples will be considered. [Work supported by NRC.]

\section{0:00-10:30 Break}

\section{0:30}

3aPA5. Scattering by tilted plastic cylinders having curved ends and truncated plastic cones. Aubrey Espana, Kyungmin Baik, and Philip L. Marston (Phys. Dept., Washington State Univ., Pullman, WA 99164-2814, marston@wsu.edu)

In prior research an acoustic backscattering enhancement was demonstrated for a bluntly truncated plastic cylinder caused by a merged caustic [F. J. Blonigen and P. L. Marston, J. Acoust. Soc. Am. 107, 689-698 (2000)]. This was confirmed with analogous light scattering experiments [P. L. Marston, Y. B. Zhang, and D. B. Thiessen, Appl. Opt. 42, 412-417 (2003)]. In recent work a different backscattering enhancement associated with a caustic was identified for tilted plastic cylinders having curved ends. When the cylinder is tilted so as to focus a shear wave at the point of internal specular reflection, the curvature of the outgoing acoustic wavefront vanishes orthogonal to the meridional plane. This was verified with analogous light scattering experiments. The flatness of the outgoing wavefront enhances the scattering. Backscattering by truncated plastic cones as a function of tilt also shows enhancements associated with the composition of the target. The time dependence of the backscattering envelope as a function of tilt reveals different features depending on whether the top or bottom of the cone is illuminated by tone bursts. [Work supported by the Office of Naval Research.]

\section{0:45}

3aPA6. Effect of changes of complex surface impedance for acoustic scattering from a wedge. Ron G. Hughes, Jan M. Niemiec (NSWC Carderock Div., W. Bethesda, MD 20817), and Herbert Überall (NSWC Carderock Div., W. Bethesda, MD 20817)

A code written earlier for the calculation of acoustic scattering from a submerged wedge has been employed in order to study the dependence of the scattering amplitude on various assumed surface impedances of the wedge. Typical wedge opening angles are $10^{\circ}$. Plane waves are assumed incident with source and observation points coinciding in the far field along the bisector of the wedge. The target strength for an impedancecoated wedge is found to lie in between those of a rigid and a pressure release wedge. A frequency region up to $20 \mathrm{kHz}$ was considered. Various numerical impedances and angles of incidence are assumed.

\section{1:00}

3aPA7. Analysis of scattering by spheres having a negative acoustical refractive index. Philip L. Marston (Phys. Dept., Washington State Univ., Pullman, WA 99164-2814, marston@wsu.edu)

Electromagnetic waves having oppositely directed phase and group velocities propagate in metamaterials having a negative permeability and negative permittivity [J. B. Pendry and D. R. Smith, Phys. Today 57(6), 37-44 (2004)]. Such materials are predicted to have unusual electromagnetic scattering properties [R. Ruppin, Solid State Commun. 116, 411-415 (2000)]. If it is possible to fabricate acoustical materials having a simultaneously negative effective elastic modulus and density (in a dynamical sense), the mechanical energy flux will have the opposite direction as the 
wave-vector associated with phase evolution. Rays descriptive of the energy flux refracted by such hypothetical materials at interfaces with ordinary fluids would be characterized by a negative acoustical refractive index. Partial-wave-series calculations of high frequency scattering by fluid spheres having an acoustical refractive index at (or close to) 1 reveal backscattering enhancements associated with glory rays which, unlike ordinary spheres [P. L. Marston and D. S. Langley, J. Acoust. Soc. Am. 73, 1464-1475 (1983)], require only a single internal chord. Generalized Lamb waves on elastic shells having opposite phase and group velocities also cause enhanced backscattering associated with unusual rays [G. Kaduchak, D. H. Hughes, and P. L. Marston, J. Acoust. Soc. Am. 96, 3704-3714 (1994)].

\section{1:15}

3aPA8. Production of evanescent acoustic waves and their scattering by resonant targets. Curtis F. Osterhoudt, Christopher Dudley, David B. Thiessen, Philip L. Marston (Dept. of Phys. and Astron., Washington State Univ., Pullman, WA 99164-2814, gardyloo@mail.wsu.edu), and Scot F. Morse (Western Oregon Univ., Monmouth, OR 97361)

An environmentally-friendly liquid mixture, denser than water and immiscible with it, and with a sound speed slower than water's, has been produced and incorporated into a scattering facility. This liquid mixture is submerged in a volume of water. In this way, the water above the denser fluid simulates a sandy ocean bottom, with the dense fluid simulating the ocean water column. When insonified by an acoustic beam from within the denser liquid, evanescent wave fields are produced in the water volume. Various targets and detectors are scanned in the water (the simulated sandy bottom) or in the mixture (the simulated ocean water column), and coupling of the evanescent waves to low-frequency resonances of cylindrical targets has been observed. Such coupling may reveal important structural characteristics of scatterers. Additionally, the complicated soundfield structure in the simulated bottom has been mapped. The structure is associated with the production of evanescent acoustic waves by a beam of sound. A model for this structure was developed using numerical wavenumber integration. [Work supported by the Office of Naval Research.]
11:30

3aPA9. Sound scattering by clusters of rigid cylinders in air. Miguel Figueres, Francisco Cervera, Andreas Håkansson, José Sánchez-Dehesa, and Jaime Llinares (Univ. Politécnica de Valencia, E-46022 Valencia, Spain, jsdehesa@upvnet.upv.es)

The scattering of sound by a cluster of rigid cylinders in air has been investigated both theoretically and experimentally. The cluster was made by a lattice of cylinders with hexagonal symmetry. The sound scattered in all directions around the cluster is measured and compared with a calculation based on multiple scattering theory. The behavior as a function of the external geometry of the cluster will also be presented. The refraction and reflection of the sound by the boundaries of the cluster is analyzed by a theoretical model that takes into account its inner crystal symmetry. [Work supported by MEC of Spain.]

11:45

3aPA10. Complete regularization of boundary integral equations in wave field diffraction problems on curved surfaces. German A. Maximov and Dmitry N. Lesonen (Dpt. 39, Moscow Eng. Phys. Inst., Kashirskoe sh. 31, Moscow, 115409, Russia, maximov@dpt39.mephi.ru)

The method of regularization of exact integral Fredgolms equations of the second kind for field or its normal derivative on the scattering surface was suggested in the previous work. It allows to obtain the stable solutions, including ones for the resonant domains, when the direct numerical solution of exact initial integral equations results to unstable solutions. Mathematically the regularization concludes in the splitting of exact integral Fredgolms equations on the equivalent system of two equations, one of them possesses the restricted by geometrically illuminated area integral operator, and other one corresponds by form to an initial one, but with diffracted source. The solution of the first equation is considered as regularized one, which coincides with exact solution in the short wavelength limit. From the physical point of view the regularization of initial integral equations concludes in their replacement on the restricted analogs, where the contributions of geometrically shadowed areas are eliminated. In the given work the regularization of the rested after splitting integral equation is represented and it is shown that the process of such regularization is auto-similar one, so that it is possible to carry out the complete regularization of the problem.

WEDNESDAY MORNING, 18 MAY 2005

REGENCY C, 8:30 TO 11:50 A.M.

Session 3aPP

Psychological and Physiological Acoustics: Hearing With More Than One Ear

\author{
Richard L. Freyman, Chair \\ Dept. of Communicative Disorders, Univ. of Massachusetts, 125 Arnold House, Amherst, MA 01003
}

Chair's Introduction-8:30

Contributed Papers

$8: 35$

3aPP1. High resolution auditory perception system. Iftekhar Alam (1013-Clover Heights, Opp Salunke Vihar, Salunke Vihar Rd., Wanowrie, Pune-411040, India [iftekharalamk@yahoo.com]) and Ashok Ghatol (Pune Inst. of Engg. and Technol. Shivajinagar, Pune, Maharashtra, India)

Blindness is a sensory disability which is difficult to treat but can to some extent be helped by artificial aids. The paper describes the design aspects of a high resolution auditory perception system, which is designed on the principle of air sonar with binaural perception. This system is a vision substitution aid for enabling blind persons. The blind person wears ultrasonic eyeglasses which has ultrasonic sensor array embedded on it. The system has been designed to operate in multiresolution modes. The ultrasonic sound from the transmitter array is reflected back by the objects, falling in the beam of the array and is received. The received signal is converted to a sound signal, which is presented stereophonically for auditory perception. A detailed study has been done as the background work required for the system implementation; the appropriate range analysis procedure, analysis of space-time signals, the acoustic sensors study, amplification methods and study of the removal of noise using filters. Finally the system implementation including both the hardware and the software 
part of it has been described. Experimental results on actual blind subjects and inferences obtained during the study have also been included.

\section{8:50}

3aPP2. Sex differences present in auditory looming perception, absent in auditory recession. John G. Neuhoff (Dept. of Psych., The College of Wooster, Wooster, $\mathrm{OH}$ 44691) and Erich Seifritz (Univ. of Bern Switzerland)

When predicting the arrival time of an approaching sound source, listeners typically exhibit an anticipatory bias that affords a margin of safety in dealing with looming objects. The looming bias has been demonstrated behaviorally in the laboratory and in the field (Neuhoff 1998, 2001), neurally in fMRI studies (Seifritz et al., 2002), and comparatively in non-human primates (Ghazanfar, Neuhoff, and Logothetis, 2002). In the current work, male and female listeners were presented with threedimensional looming sound sources and asked to press a button when the source was at the point of closest approach. Females exhibited a significantly greater anticipatory bias than males. Next, listeners were presented with sounds that either approached or receded and then stopped at three different terminal distances. Consistent with the time-to-arrival judgments, female terminal distance judgments for looming sources were significantly closer than male judgments. However, there was no difference between male and female terminal distance judgments for receding sounds. Taken together with the converging behavioral, neural, and comparative evidence, the current results illustrate the environmental salience of looming sounds and suggest that the anticipatory bias for auditory looming may have been shaped by evolution to provide a selective advantage in dealing with looming objects.

\section{9:05}

3aPP3. Lateralization of high-frequency transposed stimuli under conditions of binaural interference. Leslie R. Bernstein and Constantine Trahiotis (Dept. of Neurosci. and Dept. of Surgery (Otolaryngol.), Univ. of Connecticut Health Ctr., Farmington, CT 06030, Les@neuron.uchc.edu)

The purpose of this study was to determine whether binaural interference would occur if ITD-based extents of laterality were measured using high-frequency transposed stimuli as targets. The results of an earlier study [L. R. Bernstein and C. Trahiotis, J. Acoust. Soc. Am. 116, 30623069 (2004)], which focused on threshold-ITDs rather than extents of laterality, suggested that high-frequency transposed stimuli might be immune to binaural interference effects resulting from the addition of a spectrally-remote, low-frequency interferer. In contrast to the earlier findings, the data from this study indicate that high-frequency transposed targets can, indeed, be susceptible to binaural interference. High-frequency transposed targets, even when presented along with an interferer, yielded greater extents of ITD-based laterality than did Gaussian noise targets presented in isolation. That is, the enhanced potency of ITDs conveyed by transposed stimuli persisted even in the presence of a low-frequency interferer. Predictions made using an extension of the model of Heller and Trahiotis [L. M. Heller and C. Trahiotis, J. Acoust. Soc. Am. 99, 36323637 (1996)] accounted well for binaural interference obtained with conventional Gaussian noise targets but generally over-predicted the amounts of interference found with the transposed targets.

\section{9:20}

3aPP4. Binaural models for the detection of interaural incoherence. Matthew J. Goupell (Dept. of Phys. and Astron., Michigan State Univ., 4230 BPS Bldg., East Lansing, MI 48824) and William M. Hartmann (Michigan State Univ., East Lansing, MI 48824)

Human listeners have a remarkable ability to detect small amounts of interaural incoherence in bands of noise. Experiments using frozen noise, all with the same value of interaural coherence $(0.9922)$, show that when the bandwidth is large the ability to detect incoherence is essentially determined by the value of coherence itself. However, when the bandwidth is narrow, e.g. $20 \mathrm{~Hz}$, detection depends on some function of the moment- to-moment fluctuations in interaural phase differences and interaural level differences. Experiments using hundreds of different frozen noises and two bandwidths were performed to find the best binaural model for predicting detection performance for such narrow bands. Successful models incorporated known elements of binaural hearing-temporal averaging and compression. Models with independent processing of interaural phase fluctuations and interaural level fluctuations were marginally more successful than models based on fluctuations in lateral position. The most successful models correlated well with average human responses, $r$ $=0.89$. It seems likely that such individual fluctuations remain dominant in masking level difference experiments in the narrow band limit. [Work supported by the NIDCD grant DC 00181.]

\section{9:35}

3aPP5. A binary masking technique for isolating energetic masking in speech perception. Douglas S. Brungart, Brian D. Simpson (AFRL/ HECB, 2610 Seventh St., WPAFB, OH 45433, douglas.brungart@wpafb.af.mil), Peter S. Chang, and DeLiang Wang (Ohio State Univ., Columbus, OH 43210)

When a target speech signal is obscured by interfering speech, two distinct types of masking contribute to the resulting degradation in the intelligibility of the target talker: energetic masking caused by overlap in the time-frequency distribution of energy in the two voices, and informational masking caused by the listener's inability to correctly segregate the acoustic elements of the two voices into distinct streams. This study attempted to isolate the effects of energetic masking on multitalker speech perception with ideal time-frequency binary masks that retained those spectro-temporal regions of the acoustic mixture that were dominated by the target speech but eliminated those regions that were dominated by the interfering speech. This procedure removed the same phonetic information from the target speech that would ordinarily be lost due to energetic masking, but eliminated the possibility for the kinds of target-masker confusions that are thought to produce informational masking. The results suggest that energetic masking may play a surprisingly small role in the overall masking that occurs in certain types of multitalker speech signals. They also indicate that the number of competing talkers has a much greater influence than target-masker similarity on the amount of energetic masking that occurs in a multitalker stimulus.

\section{9:50}

3aPP6. Binaural effects in simultaneous room reflection masking. Joerg M. Buchholz (MARCS Auditory Labs., Univ. of Western Sydney, Locked Bag 1797, Penrith South DC NSW 1797, Australia)

Masked thresholds (MT) for a single test reflection masked by a direct sound (200 ms long broadband noise) were measured dependent on the time delay of the reflection for diotic as well as dichotic stimulus presentation. In the diotic case, the direct sound and the test reflection were presented equally to both ears via headphones. In the dichotic case, an ITD of $0.5 \mathrm{~ms}$ was added to the test reflection. In order to focus on simultaneous masking effects, the reflection was truncated in such a way that it formed a common offset with the direct sound. For the diotic case, the resulting data showed a MT increase with increasing reflection delay and for the dichotic case a MT decrease with increasing reflection delay, producing an intercept between both curves at a reflection delay of 6-8 ms. Hence, negative BMLDs (up to $-8 \mathrm{~dB}$ ) were found for very early reflections and positive BMLDs (up to $+8 \mathrm{~dB}$ ) for later reflections, suggesting a binaural mechanism that suppresses very early reflections and enhances later reflections. The measurement results are discussed in the background of different auditory models. 


\section{$10: 20$}

3aPP7. The influence of target-masker similarity in a three-talker diotic listening task. Nandini Iyer and Douglas Brungart (Air Force Res. Labs, 2610 Seventh St., Wright-Patterson AFB, OH 45433)

In diotic cocktail-party listening tasks, performance decreases dramatically when the number of talkers increases from two (one target and one masker) to three (one target and two maskers). In this experiment, listeners were asked to extract information from a Coordinate Response Measure (CRM) target phrase that was masked by one or two interfering utterances. The maskers were either semantically similar to the target (i.e., CRM phrases with different call signs than the target phrase) or semantically different from the target (time-reversed, contextually dissimilar, or in a different language than the target phrase). The two-talker results showed that performance was much worse when the interfering phrase was semantically similar to the target than when it was semantically different. However, given that one interfering talker was similar to the target, the contextual similarity of the second interfering talker had very little effect on performance; in other words, target intelligibility in the presence of two CRM maskers was no worse than with one CRM masker and one timereversed or foreign-language masker. These results suggest that the disruption of speech segregation caused by the addition of a second interfering talker cannot be explained solely by contextual similarity between the target and masking speech.

\section{0:35}

3aPP8. Onsets, autocorrelation functions and spikes for directionbased sound source separation. Leslie S. Smith and Dagmar S. Fraser (Dept. of Computing Sci. and Mathematics, Univ. of Stirling, Stirling FK9 4LA, Scotland, 1ss@cs.stir.ac.uk)

The aim of this work is separation of foreground speech from background sound sources using selective remixing of bandpass filtered channels. Clearly, the remixing parameters must be dynamic since the speech and noise spectra are highly non-stationary. Remixing parameters are recomputed at onsets, detected using biologically motivated techniques [L. S. Smith and D. S. Fraser, IEEE TNNS 15, 1125-1134 (2004)]. However, onsets may originate from the foreground or the background. To select appropriate onsets from the foreground source (whose direction is known) a two microphone system is used, selecting onsets for which the estimated direction in that channel corresponds to the foreground direction. Two different techniques for direction estimation are used: a channel by channel short-term autocorrelation technique, and a channel by channel spike based phase synchronous system (SBPSS), computing ITDs [L. S. Smith, in Artificial Neural Networks, Proc ICANN 2001, LNCS 2130, pp. $1103-$ 1108 (Springer, 2001)] and IIDs [L. S. Smith, in From Animals to Animats, Vol. 7, pp. 60-61 (MIT Press, 2002)]. Results comparing the performance of autocorrelation and SBPSS on single source and source plus noise signals in an office environment are presented. [Work supported by UK EPSRC.]

\section{0:50}

3aPP9. How does the mammalian brain code for binaural information? Torsten Marquardt and David McAlpine (Dept. Physiol., UCL, Gower St., London WC1E 6BT, UK)

We will summarize new physiological results which contradict established models of binaural hearing, namely (1) the A-limited range of encoded interaural time difference, ITD, (2) a correlation between ipsilateral inhibition and best ITD, and (3) a frequency dependent non-linear mapping of ITD which reflects distribution of naturally occurring ITDs. Furthermore, we will outline a new concept for binaural models which incorporates these new results, and will propose a new illustrative format in which to present the triplet of binaural parameters: interaural phase difference, interaural intensity difference, and interaural coherence (redefined).
3aPP10. Adjustment of interaural time difference in head related transfer functions based on listeners' anthropometry and its effect on sound localization. Yôiti Suzuki, Kanji Watanabe, Yukio Iwaya (R.I.E.C./G.S.I.S., Tohoku Univ., 2-1-1 Katahira, Aoba-ku, Sendai, 980-8577, Japan), Jiro Gyoba (Tohoku. Univ., Aoba-ku, Sendai, 980-8576, Japan), and Shouichi Takane (Akita. PRef. Univ., Tsuchiya, Honjo, Akita, 015-0055, Japan)

Because the transfer functions governing subjective sound localization (HRTFs) show strong individuality, sound localization systems based on synthesis of HRTFs require suitable HRTFs for individual listeners. However, it is impractical to obtain HRTFs for all listeners based on measurements. Improving sound localization by adjusting non-individualized HRTFs to a specific listener based on that listener's anthropometry might be a practical method. This study first developed a new method to estimate interaural time differences (ITDs) using HRTFs. Then correlations between ITDs and anthropometric parameters were analyzed using the canonical correlation method. Results indicated that parameters relating to head size, and shoulder and ear positions are significant. Consequently, it was attempted to express ITDs based on listener's anthropometric data. In this process, the change of ITDs as a function of azimuth angle was parameterized as a sum of sine functions. Then the parameters were analyzed using multiple regression analysis, in which the anthropometric parameters were used as explanatory variables. The predicted or individualized ITDs were installed in the nonindividualized HRTFs to evaluate sound localization performance. Results showed that individualization of ITDs improved horizontal sound localization.

\section{1:20}

3aPP11. Spatial perception of motion-tracked binaural sound. Joshua B. Melick, V. Ralph Algazi, and Richard O. Duda (CIPIC Interface Lab., UC Davis, Davis, CA 95616)

Motion-tracked binaural sound reproduction extends conventional headphone-based binaural techniques by providing the dynamic cues to sound localization produced by voluntary head motion [V. R. Algazi, R. O. Duda, and D. M. Thompson, J. Aud. Eng. Soc. 52, 1142-1156 (2004)]. It does this by using several microphones to sample the acoustic field around a dummy head, interpolating between the microphone signals in accordance with the dynamically measured orientation of the listener's head. Although the provision of dynamic cues reduces the sensitivity of the method to characteristics of the individual listener, differences between the scattered field produced by the dummy head and the scattered field that would be produced by a particular listener distorts the spatial perception. A common observation is that sound sources appear to rise in elevation when the listener turns to face them. We investigate this effect by comparing the perceived rise in elevation under three different conditions: recordings in which recordings are made using (a) the listener's own head, (b) a KEMAR mannequin, and (c) a cylindrical head with no torso. Quantitative results are presented showing the degree to which perceptual distortions are least for (a) and greatest for (c). [Work supported by NSF.]

\section{1:35}

3aPP12. Transaural virtual reality applied to front-back localization. Peter Xinya Zhang (Dept. of Phys. and Astron., Michigan State Univ., 4230 BPS Bldg., East Lansing, MI 48824) and William M. Hartmann (Michigan State Univ.)

Human sound localization can be studied by measuring head-related transfer functions for different source locations and subsequently using the data to simulate different spatial locations using headphone presentation. This technique fails to adequately simulate the difference between front and back locations because the frequencies relevant to pinna cues are so high. A transaural (cross-talk cancellation) technique has been developed that is accurate up to $16 \mathrm{kHz}$. By controlling the levels and phases of spectral components, as measured by probe microphones in the ear canals, the technique eliminates significant differences between the baseline simulation and reality. The technique has been used, with modifications to the baseline simulation, to study the front-back information content in differ- 
ent frequency bands by eliminating the information while maintaining the energy. It has also been used to test for orthogonality between front-back localization and azimuthal localization based on simple interaural differences. The technique has verified that front-back discrimination cannot be mediated by interaural differences alone. By flattening the level spectrum in one ear while leaving the other ear unchanged it has been possible to demonstrate an ear advantage for front-back discrimination. [Work supported by the NIDCD, grant DC 00181.]

WEDNESDAY MORNING, 18 MAY 2005

PLAZA B, 8:30 A.M. TO 12:00 NOON

\title{
Session 3aSA
}

\section{Structural Acoustics and Vibration: Approximation Techniques for Fluid Loading Effects I}

\author{
David Feit, Cochair \\ Applied Physical Sciences, Inc., 2 State St., New London, CT 06320 \\ Joel M. Garrelick, Cochair \\ Cambridge Acoustical Associates/ETC, 84 Sherman St., Cambridge, MA 02140
}

Invited Papers

8:30

3aSA1. The rational function approximation for fluid loaded vibrating structures. David Feit (Appl. Physical Sci. Corp., Ste. 300, 2 State St., New London, CT 06320)

A complicating feature of fluid-structure interaction problems is the non-local or long-range effect of the fluid loading acting on a structure vibrating in response to time-harmonic loading while submerged in an acoustic medium. DiPerna and Feit [J. Acoust. Soc. Am. 114, 194-199 (2003)] recently introduced an approximation for this effect using a rational function approximation (RFA) to the spectral impedance. The latter relates the transform of the surface pressure to that of the surface velocity, and transforming back to the physical domain gives rise to a high-order differential pressure-velocity relationship. The coefficients of the nth order differentials are inversely proportional to the nth order of frequency so that the approximation gets better with increasing frequency. The approximation originally developed for a planar vibrating surface, has now been extended to a cylindrical surface, and this development and several applications of the RFA technique are presented. [Work supported by the Carderock Division Naval Surface Warfare Center Intra-laboratory Independent Research (ILIR) program and the Office of Naval Research.]

\section{9:00}

3aSA2. Transmission line analysis of cylindrical shells with exterior fluid loading. Ann Stokes, Charles Corrado, and Joel Garrelick (Appl. Physical Sci., Inc., 2 State St., Ste. 300, New London, CT 06320)

The Wave Transmission Line (WTL) numerical modeling formulation has been developed to understand and efficiently compute the transmission of acoustic energy through fluid-filled elastic shells. The structural systems are represented as a sequence of components and discontinuities connected with straight duct sections. The motion of straight segments is described in terms of amplitudes of freely traveling, left-and-right propagating waves. Recent work in Rational Function Approximation (RFA) of exterior fluid loading permits this approach to be extended to cylindrical structures with interior and exterior fluid loading. The resulting approach is more computationally efficient than conventional finite element approaches. This paper describes development of systems of transmission line equations for segmented cylindrical shells with exterior and interior fluid loading.

9:30

3aSA3. The Fresnel-Product Approximation, and comments on extending rational function approximation to include flow. Rudolph Martinez and Carina Ting (Anteon Corp., 84 Sherman St., Cambridge, MA 02140)

The first half of this presentation reviews the existing development of the Fresnel-Product Approximation as a replacement of the exact fluid impedance on a two-sided blade-like object. Excellent performance versus exact calculations is demonstrated for vibration patterns above, below, and at coincidence, viz., for flexural wavelengths that are longer, shorter, or equal to that of characteristic sound. The approximate solution is global and thus of the WKB type: (1) It captures the correct edge behavior of diffraction loads on the vibrating blade; (2) Collapses to the Kirchhoff solution away from both edges at high frequencies; and (3) Differs by only a small factor when applied at low frequencies to predict the blades globally entrained fluid mass. The second half of the talk addresses the Rational Function Approximation (RFA) pioneered by Feit and DiPerna. It shows how their spectral technique could be readily generalized to add flow effects, including the influence of shed vorticity as it co-exists with sound. Flowless acoustics will be derived from fully unsteady aerodynamics/aeroacoustics through a delicate limiting process and an integration by parts. The talk ends with a discussion of how current applications of the RFA could be stripped of some of their structural-acoustic artifacts. 
3aSA4. Rational approximations of surface impedance operators on curved surfaces with application to high-frequency scattering by thin shells. David C. Calvo (Acoust. Div. Naval Res. Lab., Washington, DC 20375-5320)

In the analytical solution of scattering and radiation problems on curved surfaces using Fourier transforms, surface impedance functions featuring ratios of Hankel functions arise. Recently, an approach of approximating the impedance by a rational function was introduced in which the squared transform variable is replaced by a differential operator. By taking into account the impedance function poles in the rational approximation, an accurate and efficient numerical scheme is obtained applicable to wide-frequency ranges and wide-scattering angles where creeping waves are important. The rational approximation and operator introduction in effect creates an inverse Fourier transform operator so explicit residue calculus inversion is not needed if numerical results are desired. For general convex surfaces, the approximate impedance operator is a wide-angle on-surface radiation condition operator with more accuracy than the narrow-angle one of Kriegsmann et al. The use of rational approximations resembles that of Collins et al. for the square-root function in ocean acoustics and DiPerna \& Feit for the flat plate, the important difference being the curvature. Results of using this approach for two and three-dimensional scattering problems will be presented along with recent application to scattering by thin cylindrical and spherical shells at high frequencies. [Work supported by ONR.]

\section{Contributed Papers}

\section{0:45}

3aSA5. Well- and ill-considered numerical solution constructions. John J. McCoy (The Catholic Univ. of America, Washington, DC 20064)

One requirement for a prediction problem to be well posed is that its solution must not be unduly sensitive to small changes in the problem specification. A similar requirement can be demanded for the numerical construction of the solution of a well-posed problem to be well considered; the achieved solution must not be unduly sensitive to small numerical errors that are unavoidable in the construction's implementation. Illconsidered constructions typically result on reformulating a well-posed problem, now as a series of sub problems, one of which is ill posed. Three classes of potentially ill-considered constructions of the steady state vibration field in a bounded continuous structure and of the narrow band acoustic field in an extended propagation environment appear prominently in the literature. One class is solution constructions based on "marching." A second class is solution constructions based on "substructuring" the computational domain. A third class is solution constructions that are based on a Born-series representation. Examples of well- and ill-constructions for each of the classes are briefly described, with well- and ill-considered marching solutions discussed in some detail.

\section{1:00}

3aSA6. Elastoacoustic response of laminated plates. Ramesh Kolar (Naval Postgrad. School, Mech. \& Astro Eng., Code ME/Ko, Monterey, CA 93943)

The application of composite materials in the aerospace and naval structures has increased enormously due to high specific strength and specific stiffness afforded by these materials. In this paper a formulation is developed based on Hamilton's Principle and laminated composite plate theory to study the elasto-acoustical response of composite plates under heavy fluid loadings. The formulation starts by using Hamilton's principle in conjunction with shear deformable theory of laminated composite plates. The acoustic pressure described by wave equation is computed similar to Sandman and Nelisse. Using the Rayleigh Ritz method and symbolic mathematics for evaluation of integrals, the formulation provides efficient approach for the problem defined. Typical results include radiation impedance as a function of driving frequency, vibroacoustic indicators such as radiated sound power and mean square velocity for a model problem. Such results are very important in studying constrained layer damping when viscolastic dampers are used in structural components.

\section{1:15}

3aSA7. Prediction of radiation from finite-length fluid-loaded periodic structures subject to broadband excitation using local-global homogenization. Pavel V. Danilov and Donald B. Bliss (Mech. Eng. and Mater. Sci., Duke University, Durham, NC 27708, dbb@duke.edu)

Local-Global Homogenization (LGH) is a method to predict directly the smooth global response of periodic fluid-loaded structures in a selfcontained manner. Many fluid-loaded structures, such as fuselages and hulls, have periodically spaced braces, ribs, or attachments. Structural motion, acoustic radiation, and the interior sound field are of interest. Calculating the motion of such fluid-loaded structures is difficult because of their complexity and the disparity of length scales. Periodic discontinuities cause the structural response to occur in a broad spectrum of spatial wavenumbers, and to exhibit stop-band and pass-band behavior. The broad spatial wavenumber spectrum contains both radiating and non-radiating components. The global low-wavenumber part of the response is most efficiently coupled to the acoustic field, since low wavenumbers correspond to supersonic phase speeds. In the LGH reformulation, an infinite order operator that embodies both the structural modes and the evanescent component of the fluid loading governs the equivalent smooth global problem. Numerical implementation is demonstrated for the structural response and acoustic radiation from a finite-length fluid-loaded plate with periodic impedance discontinuities subject to broadband excitation. Calculations show good agreement with the exact solution and substantially improved computational efficiency. Radiation characteristics are discussed for single frequency and broadband structural motions.

\section{1:30}

3aSA8. Vibroacoustic optimization of skin damped structures for noise reduction. Lionel Zoghaib (Lab. de Mécanique et d'Acoustique CNRS, Marseille, France, zoghaib@lma.cnrs.mrs.fr)

Most of the time the widely used passive treatments are designed to obtain the highest achievable damping. Although this might be satisfactory from a vibrational point of view the approach is not optimal for noise reduction. The idea is illustrated in the case study of a plate on which polymer/aluminum thin patches have been bonded. The system acoustic pressure and structure displacement equations are transformed into a single integro differential one governing the plate displacement. The solution is then expanded into a series of resonance modes. These modes are estimated using a perturbation expansion with respect to the fluid/solid density ratio. Numerically, in vacuo resonance modes are firstly computed using a viscoelastic incompressible 3D FE program together with a Newton-type iterative method and a sparse complex matrix solver implementing ARPACK. Radiation modal impedances are then computed to ponderate the in vacuo solution. The numerical model has been experimentally validated. Its specificity lies in the separate computation of three damping components: thermoelastic, skin and acoustic damping, this last 
one being caused by the fluid/structure coupling. Finally an optimization process determines an optimal system configuration to achieve the lowest acoustic damping while obtaining the highest viscoelastic damping.

\section{1:45}

3aSA9. An impedance modeling technique of a fluid-loaded structure. Chih-Chun Cheng and Pi-Wen Wang (Dept. of Mech. Eng., Natl. Chung Cheng Univ., 160, San-Hsing, Ming-Hsiung, Chia-Yi 621, Taiwan)

A methodology of modeling the fluid-loaded structures using the impedance technique is proposed. The structural response and the fluid loading effect are expressed in terms of structural impedance and the acoustic wave impedance. Then a formulation that assembles the acoustic imped- ance and the structural impedance is derived and can be used to determine the response of a fluid-loaded structure. The advantage is simply that the impedance can be obtained either experimentally or analytically and then the response of the fluid-loaded structure can be found using the proposed impedance coupling formula without deriving the equation of motion which usually encounters difficulties when the structure is in an irregular shape or the associated boundary conditions are complicated. Two numerical examples are presented. The first is to validate that the fluid loading calculated using the proposed methodology is the same as that from the traditional Fourier Transformation method. The second is to demonstrate the potential of this proposed method applied in analyzing an active material system which is commonly used in the vibro-acoustic control. [Work supported by NSC of Taiwan.]

WEDNESDAY MORNING, 18 MAY 2005

REGENCY D, 8:00 A.M. TO 12:00 NOON

\title{
Session 3aSC
}

\section{Speech Communication: Indigenous Languages of North America (Lecture/Poster Session)}

\author{
Sonya F. Bird, Chair \\ Dept. of Linguistics, Univ. of Victoria, Victoria, BC V8W 3P4, Canada
}

Chair's Introduction-8:00

Invited Papers

8:05

3aSC1. Phonetic study of North American languages history and results. Ian Maddieson (Dept of Linguist., Univ. of California, Berkeley, CA 94720)

Serious phonetic study of North America languages started at the beginning of the 20th century. Within limits of available technology, aspects of speech articulation, aerodynamics and acoustics were investigated. One clear motivation was to understand how classes of sounds unfamiliar from study of better-known European and Asian languages were produced. Glottalized consonants and stops then referred to as "intermediate" (voiceless unaspirated) received particular attention. Nasal airflow, lip position in vowels, and tone and pitch accent were also investigated. Significant insights on relative timing were obtained, inter alia foreshadowing VOT measurement as a useful discriminator of laryngeal activity and revealing part of the mechanism by which ejective consonants are made. After the 1920's, the idea of "psychologically real" phonemes which ignored phonetic differences became the ruling paradigm in American linguistics, contributing to a decline of interest in phonetic studies that basically lasted until around the 1980's. When interest renewed, a new vision that phonetic patterns show regularities independent of phonemic structure guided research, and considerable attention was also paid to how indigenous American languages fit into overall phonetic typologies. Recent work is also often informed by concern for documentation of endangered languages and community interest in language revival.

3aSC2. Pitch accent timing in Chickasaw. Matthew K. Gordon (Dept. of Linguist., Univ. of California, Santa Barbara, Santa Barbara, CA 93106)

This paper examines the temporal realization of pitch accents in Chickasaw, a Muskogean language of Oklahoma. Questions in Chickasaw have a Lat their right edge preceded by a $\mathrm{H}^{*}$ pitch accent that docks on one of the final three syllables of the last word in the Intonational Phrase: on a final CVV, otherwise on a heavy (CVV or CVC) penult, otherwise on the antepenult. Three speakers of Chickasaw were recorded uttering a list of words varying in the location of the pitch accent and the type of syllable carrying the pitch accent. Results indicate a tendency to realize the pitch accent relatively late in non-final pitch accented syllables (during the last 25 and during the coda consonant following a short vowel). However, final accented syllables realize the pitch accent early (within the first 20 vowel) and also display substantial vowel lengthening (by over 50 vowels in the penult or antepenult). These results suggest that pressure to avoid tonal crowding between the pitch accent and boundary tone overrides the late realization of pitch accents characteristic of positions in which crowding is less of an issue. 
3aSC3. How the pharyngeal resonator is controlled articulatorily. John H. Esling (Dept of Linguist., Univ. of Victoria, Victoria, BC, Canada V8W 3P4, esling@uvic.ca)

Indigenous languages of North America, especially west coast Vancouver Island Nuuchahnulth and Interior BC Salish, illustrate an extensive range of use of the pharyngeal resonator. The pharynx is classified as part of the laryngeal articulator (rather than lingual) because the laryngeal constrictor mechanism (controlling changes from the glottis to the aryepiglottic folds) is the principal articulator whose movements determine the shape of the pharynx. The Nuuchahnulth (Nootka) dialects (e.g., Ahousaht) and Thompson, MosesColumbia, and Spokane/Kalispel Salish varieties all close the larynx completely for the speech sound epiglottal stop. With the airway optimally shut, the pharynx is small; the aryepiglottic folds are pressed against the epiglottic tubercle, the tongue is retracted, and the larynx is raised. There are good indications that the thyroarytenoid, aryepiglottic, thyroepiglottic, hyoglossus and hyothyroid muscles participate in this buckling maneuvre. These languages also contain either a pharyngeal fricative or approximant (at the same place of articulation but with less stricture) and, in Thompson, also pharyngealized uvulars (with even less stricture). Pharyngeal resonance in all of these cases is dependent initially on the shortening of the supraglottic tube and subsequently on the combined effect of tongue retraction and larynx raising reducing the volume immediately above the supraglottic tube.

9:20

3aSC4. One hundred years of instrumental phonetic fieldwork on North America Indian languages. Joyce McDonough (Dept. of Linguist., Univ. of Rochester, Rochester, NY 14627)

A resurgence of interest in phonetic fieldwork on generally morphologically complex North American Indian languages over the last 15 years is a continuation of a tradition started a century ago with the Earle Pliny Goddard, who collected kymographic and palatographic field-data between 1906-1927 on several Athabaskan languages: Coastal Athabaskan (Hupa and Kato), Apachean (Mescalero, Jicarilla, White Mountain, San Juan Carlos Apache), and several Athabaskan languages in Northern Canada (Cold Lake and Beaver); data that remains important for its record of segmental timing profiles and rare articulatory documentation in then largely monolingual communities. This data in combination with new work has resulted in the emergence of a body of knowledge of these typologically distinct families that often challenge notions of phonetic universality and typology. Using the Athabaskan languages as benchmark example and starting with Goddard's work, two types of emergent typological patterns will be discussed; the persistence of fine-grained timing and duration details across the widely dispersed family, and the broad variation in prosodic types that exists, both of which are unaccounted for by phonetic or phonological theories.

\section{9:45}

3aSC5. Coronal ejectives in Ahtna Athabaskan. Siri Tuttle (Univ. of Alaska, Fairbanks, AK 99775)

Ahtna, a non-tonal Alaskan language, is one of few Athabaskan languages that has retained word-final ejectives. The loss of stem-final glottal stops and ejectives in the other languages is correlated with tonogenesis. Hargus (2004) finds voice quality distinctions (creakiness, variability, and increased energy in higher harmonics) preceding stem-final glottal consonants in Witsuwiten, another nontonal Northern language. The present study considers the acoustics of stem-initial and stem-final [t'] and [ts'] in Central and Lower Ahtna. Stem-initial ejectives are canonically shaped, with defined silent period following oral release. In stem-final position, as predicted by Kari (1990), variable non-ejective realizations are found in both word-final and intervocalic (suffixed) position. These realizations are accompanied by variable voice quality effects in preceding vowels, more frequent and pronounced than those found variably in vowels following stem-initial ejectives. Stem-final [ $\left.\mathrm{t}^{\prime}\right]$ is frequently pronounced as glottal stop, and final [ts'] as ['s] where $<,>$ stands for glottal stop. Strikingly, non-ejective realizations of stem-finals are found in suffixed and nonsuffixed pairs with the same stem, suggesting that stem-final effects generalize to all tokens of stems. These findings support Leers (1979) picture of spirantization and suprasegmentalization in Athabaskan tonogenesis, as glottal effects gravitate to nucleus position.

\section{0:10-10:25 Break}

\section{0:25}

3aSC6. Tone and prosodic organization in Cherokee nouns. Keith Johnson (Dept. of Linguist., 1203 Dwinelle Hall, UC-Berkeley, Berkeley, CA 94720-2650) and Marcia Haag (Norman, OK 73019-2032)

Preliminary observations in the speech of one speaker of Cherokee led us to postulate three factors affecting tone in Cherokee. (1) Tone may be lexically specified with distinctive low, low fall, low rise, and high tones. (2) There is a metrically determined high fall pattern which may be distributed over not more than 2 syllables from the right edge of a prosodic domain. (3) Intonational domains may be associated with discourse functions, marked by high fall, or by pitch range upstep. This paper tests these observations in recordings of word lists and sentences produced by five additional speakers. The analysis we give, positing both lexical tone and metrical prosodic accent, is not unique in descriptions of language, but is different from the usual description of Cherokee. [Work supported by NSF.] 


\section{Contributed Papers}

All posters will be on display and all authors will be at their posters from 10:50 a.m. to 12:00 noon.

3aSC7. Harmony in Gitksan. Noriko Yamane-Tanaka and Atsushi Fujimori (Dept. of Linguist., Univ. of University, E270-1866 Main Mall, Vancouver, BC, Canada V6T 1Z1)

What types of consonants are transparent in vowel harmony in Gitksan has been little understood. Previous studies suggest that it is translaryngeal harmony, where the qualities of unstressed suffixal vowels are harmonized with the ones of last stem vowels, apparently skipping the stem-final laryngeal stop and laryngeal fricative [Rigsby, unpublished manuscript, University of Queensland, Australia, 1986; Rigsby and Ingram, International Journal of American Linguistics 56, 251-263 (1990)]. However, our original data show that harmony takes place when the stem-final consonant is uvular as well. It suggests that it cannot simply be analyzed as laryngeal transparency, but it could be reanalyzed as [+low] transparency. The present study examines cross-consonantal vowel harmony through acoustic experiment. It is predicted that there is a significant difference in formant values between the vowels across uvular or laryngeal consonants versus the ones across the other consonants. New data from an Eastern Gitksan speaker (female, 72) and its analysis will be presented. Implications for locality of vowel harmony as well as for the phonological treatment of consonantal transparency will be discussed. [Work supported by SSHRC.]

3aSC8. The effect of $[h]$ on tonal development in Nahuatl. Susan G. Guion (Linguist. Dept., 1290 U. of Oregon, Eugene, OR 97403, guion@uoregon.edu) and Jonathan D. Amith (Gettysburg College, Gettysburg, PA 17325)

In both its classical and modern forms, Nahuatl is usually described as having penultimate accent (with little discussion as to whether this is a phrase- or word-level phenomena). However, some varieties of Mexican Guerrero Nahuatl have recently been described as having word accent in locations related to the position of a historical non-word-final coda $* h$. Here, the hypothesis is investigated that coda $* h$ lowered the fundamental frequency $(F 0)$ of the tautosyllabic vowel and that a $H-L$ pitch contour was subsequently incorporated into the lexical representation of the word as $* h$ was weakened and lost. Recordings were made of four dialects at varying stages of $* h$ loss and tonal innovation. Six speakers from each dialect were recorded producing words in phrase medial and phrase final positions, some with historical coda $* h$ and some without, and the $F 0$ of each syllable in these words measured. The results indicated that the new accentual patterns found in some dialects have their origin in the phonetic lowering of $F 0$ in syllables containing coda $* h$. It also seems that once a new accentual pattern is developed and the $* h$ lost, the resulting phonologized pattern may not correspond to the phonetic locus of the sound change.

3aSC9. Glottalization cues of Nuu-chah-nulth glottalized resonants. Marion G. Caldecott (Dept. of Linguist, Univ. of British Columbia, Canada V6T 11, mariongc@interchange.ubc.ca)

Theories of positional neutralization (Steriade, 1997)] predict that preglottalized resonants should not occur word-initially because the cues to glottalization are not salient. Contrary to this, Nuu-chah-nulth, a Wakashan language, has pre-glottalized that do occur in word-initial position. This paper will show that a severely truncated resonant portion is the cue to glottalization in resonants in Nuu-chah-nulth. Previous research [Carlson et al., J. Jpn. Int. Prop. Assoc. 31, 275-279 (2001)] shows that Nuu-chahnulth glottalized resonants consist of a stop closure that is phonetically identical to phonemic glottal stop followed by a (mostly) plain resonant. That study also found that glottalized resonants are twice as long in initial position as plain resonants. Following up on this, a pilot study [Caldecott and Kim, presented at the 1st annual Wakashan Linguistics Conference,
2004] indicated that the length of the resonant portion in glottalized resonants was in fact half that of a normal resonant. This paper expands on the previous study, incorporating new acoustic data on resonants in all word positions. Data are elicited from a fluent native speaker and measured using Praat. [Work supported by SSHRC.]

3aSC10. Glottalized resonants in NLe kepmxcin (Thompson River Salish). Patricia A. Shaw, Fiona M. Campbell (Dept. of Linguist., Univ. of British Columbia, E270-1866 Main Mall, Vancouver, BC, Canada V6T 1Z1), and Flora Ehrhardt (Lytton Indian Band, BC)

This study examines properties of glottalized resonants (/m', n', l', y', w'/) in NLe?kepmxcin, a Northern Interior Salish language, both comparing them with their plain counterparts $(\mathrm{m}, \mathrm{n}, \mathrm{l}, \mathrm{y}, \mathrm{w})$ and considering contextual factors as defined by word, syllable, and prominence positions. Despite the relative cross-linguistic rarity of glottalized resonants, they are of considerable theoretical interest given that several recent studies [e.g., Bird and Caldecott, Proceedings of the 10th AICSST (in press)] indicate that their articulatory and acoustic realization may vary significantly across languages and contexts. Of particular relevance to the present study is Carlson, Esling, and Harris' [UMOPL 17, 58-71 (2004)] claim that in NLe?kepmxcin "glottalized resonants are a sequential combination of the resonant plus [glottal stop]" [pg. 64]. Based on the analysis of glottalized resonants in this study, it appears that the picture is more complex than consistent post-glottalization. Of special interest are glottalized resonants in word-initial position, both because this class is seriously underrepresented in the previous literature, and because its characteristics challenge Carlson, Esling, and Harris' sequencing generalization. Understanding the organization of glottalized resonants will help clarify both sequential and markedness issues in NLe?kepmxcin phonology. [Work supported by SSHRC.]

3aSC11. Phonetic structures of Washo. Patrick J. Midtlyng and Alan C. L. Yu (Dept. of Linguist., Univ. of Chicago, 1010 E 59 St., Chicago, IL 60637, aclyu@uchicago.edu)

Washo is a severely moribund language spoken in several townships near the California-Nevada border southeast of Lake Tahoe. The Washo language is now used only by approximately 13-20 elderly speakers with very few fluent speakers under 60. Recordings were collected in Dresslerville, Nevada from two native speakers. Recordings collected by William Jacobsen Jr. in the 1960s provided additional data for comparison. Analysis showed that all the stops were either voiceless unaspirated, voiceless aspirated, or ejectivized. Vowel analyses showed the nature of the six long and short vowels. Special attention was paid to the nature of vowels and consonants in and around the stressed syllable. Post-tonic sibilants and sonorants are lengthened intervocalically even though consonantal length is not contrastive in Washo. Vowel length contrasts only in the stressed syllable. Stressed vowels are always long before an intervocalic voiceless unaspirated stop, but vocalic length contrast remains before aspirated stops and ejectives.

3aSC12. An ultrasonic investigation of retracted consonants in St'at'imcets (Lillooet Salish). Nahal Namdaran (Dept. of Linguist., Univ. of British Columbia, 1866 Main Mall, Buchanan E270, Vancouver, BC, Canada V6T 1Z1)

Tongue retraction is rare in native languages of North America. Previous literature on St'at'imcets (also known as Lillooet) has left unanswered the question of how retracted consonants (uvulars, a pharyngeal, and a subset of retracted coronals) are articulated, and whether they in fact involve tongue retraction. Based on perceptual and acoustic data, previous descriptions differ with respect to the involvement of the tongue dorsum 
and tongue root in the articulation of St'at'imcets retracted consonants, particularly with the orthographic "g," disputed to be either a uvular or pharyngeal approximant [Shahin, 1997; van Eijk, 1997]. Articulatory data of retracted and nonretracted consonants were collected from 4 St'at'imcets speakers via a portable ultrasound machine. Results indicate a significant difference in tongue root, dorsum, and body position for retracted versus nonretracted consonants, with the former possessing more tongue root and dorsum backing and tongue body lowering than the latter. Furthermore, results show "g" to be articulated similarly to the uvular consonant, with the tongue dorsum moving towards the posterior uvula/ upper pharyngeal region of the vocal tract. These results provide direct evidence for the articulatory makeup of St'at'imcets retracted consonants and shed light on St' at'imcets retraction effects and phonological harmony processes. [Work supported by SSHRC.]

3aSC13. An acoustic study of Deg Xinag fricatives. Richard Wright, Sharon Hargus, and Julia Miller (Dept. of Linguist., Univ. of Washington, Box 354340, Seattle, WA 98195-4340)

Few studies have looked at the acoustic properties of fricatives in Native American languages. Notable exceptions are McDonough (2003) on Navajo, and Gordon, Barthmaier, and Sands (2002), which examined a variety of languages, including several Native American ones. In Deg Xinag, an endangered Athabaskan language spoken in Alaska, there are eight places of articulation for voiceless fricatives [a relatively large phonetic inventory, according to Maddieson (1984)], including some rarely studied place contrasts (e.g. alveolar versus retroflex). In this study, preand post-vocalic fricatives were digitally recorded in the field from eight speakers (two males, six females) using a head-mounted mic to control for distance from the source. The segmental context was also controlled for, the neighboring vowel being [a] in all cases. Each speaker produced four repetitions of each word. The first four spectral moments, lowest spectral peak, and the three loudest spectral peaks were measured at the midpoint of each fricative, and each speaker's average for each of the measures was calculated. In this poster, qualitative results in the form of spectrographic analysis will be presented. Repeated measures ANOVA for each of the quantitative measures will also be presented. [Work supported by NSF.]

3aSC14. Glottalized consonants in Oaxaca Chontal. Heriberto Avelino and Ian Maddieson (Dept of Linguist., Univ. of California, Berkeley, CA 94720)

Lowland Oaxaca Chontal, a language generally classified as Hokan and hence related to Yuman and Pomo languges, is now spoken by only a small percentage of the Chontal people who live on the Pacific coast of Oaxaca state, Mexico. Recordings were made of seven speakers in their 60's to 80's in December 2003. The language has a series of glottalized obstruents some of which which vary in production between fricative and affricate realizations. Our data show the labio-dental and lateral are the most likely to have fricative realizations, the coronals are regularly affricates and the velar is regularly a stop. These segments are contrastive but morphologically related to the voiceless fricatives of the language. This is demonstrated by one common plural formation process which adds glottalization to a final consonant (e.g. /apix/ "stone," /apik'/ "stones"). After a vowel the same morpheme is realized as a glottal stop (e.g. /u/ "eye," /u/ "eyes"). However, not all sequences of voiceless fricative + glottal stop are realized as glottalized obstruents (e.g. /ase/ "atole"). Chontal also has glottalized nasals and approximants. As in other languages the accompanying glottal constriction is variable in degree and timing.
3aSC15. Consonantal perturbations of pitch in Halkomelem Salish. Jason Brown and James J. Thompson (Dept. of Linguist., Univ. of British Columbia, 1866 Main Mall, Buchanan E270, Vancouver, BC, Canada V6T 1Z1, jcb@interchange.ubc.ca)

It has long been noted that consonants have an effect on the pitch of a following vowel: voiceless stops tend to raise $F 0$, while voiced stops lower it. It has also been suggested that the duration of such perturbations is shorter in tone languages than in non-tone languages [Hombert, Studies in African Linguistics (1977)]. This study compares the effects that consonants have on $F 0$ in two closely related Salish languages: Island Halkomelem, a non-tone language, and Upriver Halkomelem, a language that has reportedly undergone some limited tonogenesis but offers no clear prosodic clues regarding tonality. The effects of the voiceless and ejective stop series were observed, and measurements of $F 0$ were taken at the onset of voicing for the vowel, then at $20 \mathrm{msec}$. intervals up to $100 \mathrm{msec}$. Preliminary results indicate that i) Island Halkomelem shows a greater magnitude of difference in $F 0$ at vowel onset between the voiceless and ejective stops than Upriver Halkomelem, and ii) Island Halkomelem shows greater durations of consonantal perturbations of $F 0$ than does Upriver Halkomelem. This suggests that Upriver Halkomelem may have become more sensitive to pitch than the Island dialect, supporting the interpretation of this language as tonal. [Work supported by Phillips Fund.]

3aSC16. Acoustic cues to Nehiyawewin constituency. Clare Cook and Jeff Muehlbauer (Univ. of British Columbia, Dept. of Linguist., 1866 Main Mall, Buchanan E270, Vancouver, BC, Canada V6T 1Z1, clarec@ interchange.ubc.ca)

This study examines how speakers use acoustic cues, e.g., pitch and pausing, to establish syntactic and semantic constituents in Nehiyawewin, an Algonquian language. Two Nehiyawewin speakers autobiographies, which have been recorded, transcribed, and translated by H. C. Wolfart in collaboration with a native speaker of Nehiyawewin, provide naturalspeech data for the study. Since it is difficult for a non-native-speaker to reliably distinguish Nehiyawewin constituents, an intermediary is needed. The transcription provides this intermediary through punctuation marks (commas, semi-colons, em-dashes, periods), which have been shown to consistently mark constituency structure [Nunberg, CSLI 1990]. The acoustic cues are thus mapped onto the punctuated constituents, and then similar constituents are compared to see what acoustic cues they share. Preliminarily, the clearest acoustic signal to a constituent boundary is a pitch drop preceding the boundary and/or a pitch reset on the syllable following the boundary. Further, constituent boundaries marked by a period consistently end on a low pitch, are followed by a pitch reset of $30-90 \mathrm{~Hz}$ and have an average pause of 1.9 seconds. I also discuss crossspeaker cues, and prosodic cues that do not correlate to punctuation, with implications for the transcriptional view of orthography [Marckwardt, Oxford 1942].

3aSC17. Discourse-level contours in Nehiyawewin. Jeff Muehlbauer and Clare Cook (Univ. of British Columbia, Dept. of Linguist., 1866 Main Mall, Buchanan E270, Vancouver, BC, Canada V6T 1Z1, jefmuehl@interchange.ubc.ca)

This study describes declination and discourse-sized intonation contours in Nehiyawewin, an Algonquian language whose pitch and intonation systems have not been previously studied. The study draws on 270 min of recordings of two female Nehiyaw elders telling their life stories to another Nehiyawewin native speaker. Data is analyzed by using Praat's default algorithm to generate $f 0$ curves for each breath group. Preliminary results indicate: (1) When breath-group internal pitch peaks are considered, an obvious downward trend of $f 0$ occurred in fewer than half the breath groups analyzed (about $40 \%$ or $37 / 90$ ). This raises questions about the role of classical declination in natural discourse [Umeda, Journal of 
Phonetics 10 (1982)]. (2) When we abstract away from declination within a breath group by computing mean $f 0$ and mean pitch peak for each breath group and tracking trends for these means, larger patterns seem to emerge; breath groups can be grouped into larger units based on raising and lowering trends. These units have a mean peak range of $150 \mathrm{~Hz}$ with a $30 \mathrm{~Hz}$ change from one breath group to the next and correspond to a domain of around five clauses (about 3-4 breath groups, about 45 syllables).

WEDNESDAY MORNING, 18 MAY 2005

GEORGIA A, 9:00 A.M. TO 12:00 NOON

\title{
Session 3aSP
}

\section{Signal Processing in Acoustics: Potpourri}

\author{
David M. Fromm, Chair \\ Naval Research Laboratory, 4555 Overlook Ave., SW, Washington, DC 20375-5350
}

\section{Contributed Papers}

\begin{abstract}
9:00
3aSP1. Wavelet based detection of manatee vocalizations. Berke M. Gur and Christopher Niezrecki (Dept. of Mech. Eng., Univ. of Massachusetts Lowell, One University Ave., Lowell, MA 01854-2881, Chris_Niezrecki@uml.edu)

The West Indian manatee (Trichechus manatus latirostris) has become endangered partly because of watercraft collisions in Florida's coastal waterways. Several boater warning systems, based upon manatee vocalizations, have been proposed to reduce the number of collisions. Three detection methods based on the Fourier transform (threshold, harmonic content and autocorrelation methods) were previously suggested and tested. In the last decade, the wavelet transform has emerged as an alternative to the Fourier transform and has been successfully applied in various fields of science and engineering including the acoustic detection of dolphin vocalizations. As of yet, no prior research has been conducted in analyzing manatee vocalizations using the wavelet transform. Within this study, the wavelet transform is used as an alternative to the Fourier transform in detecting manatee vocalizations. The wavelet coefficients are analyzed and tested against a specified criterion to determine the existence of a manatee call. The performance of the method presented is tested on the same data previously used in the prior studies, and the results are compared. Preliminary results indicate that using the wavelet transform as a signal processing technique to detect manatee vocalizations shows great promise.
\end{abstract}

\section{9:15}

3aSP2. Background noise cancellation for improved acoustic detection of manatee vocalizations. Zheng Yan (Dept. of Mech. and Aerosp. Eng., Univ. of Florida, Gainesville, FL 32611-6250), Christopher Niezrecki (Univ. of Massachusetts Lowell, Lowell, MA 01854-2881, Chris_Niezrecki@uml.edu), and Diedrich O. Beusse (Univ. of Florida, Gainesville, FL 32610-0126)

The West Indian manatee (Trichechus manatus latirostris) has become endangered partly because of an increase in the number of collisions with boats. A device to alert boaters of the presence of manatees, so that a collision can be avoided, is desired. Practical implementation of the technology is dependent on the hydrophone spacing and range of detection. These parameters are primarily dependent on the manatee vocalization strength, the decay of the signal strength with distance, and the background noise levels. An efficient method to extend the detection range by using background noise cancellation is proposed in this paper. An adaptive line enhancer (ALE) that can detect and track narrowband signals buried in broadband noise is implemented to cancel the background noise. The results indicate that the ALE algorithm can efficiently extract the manatee calls from the background noise. The improved signal-to-noise ratio of the signal can be used to extend the range of detection of manatee vocalizations and reduce the false alarm and missing detection rate in their natural habitat.

\section{$9: 30$}

3aSP3. Electronic noise suppression in the wavelet, frequency, and time domains. Juan Arvelo, Jr. (Appl. Phys. Lab., Johns Hopkins Univ., 11100 Johns Hopkins Rd., Laurel, MD 20723-6099)

Automatic machine gun recordings were severely contaminated with electronic noise. A detailed examination of the character of the electronic noise revealed that it consists of periodic pulses at an interval of about 4.2 $\mathrm{ms}$ and that its frequency content is rich in narrow-band lines with a colored broadband background. Given the impulsive nature of the electronic noise in the time domain, wavelet decomposition was applied in an attempt to harness the noise and to null it out. An alternative approach was also implemented where a bank of stop band Butterworth filters were applied in the frequency domain to suppress the narrow-band components of the electronic noise. Other approaches include the use of adaptive cancellation in the time and frequency domains. We will demonstrate and compare the performance of these approaches emphasizing the need to minimize their influence in distorting the signal of interest.

\section{9:45}

3aSP4. Maximum entropy method for constructing environmental parameter probability density functions. Leon $\mathrm{H}$. Sibul, R. Lee Culver, David L. Bradley, and H. John Camin (Appl. Res. Lab. and Grad. Program in Acoust., P.O. Box 30, State College, PA 16804, rlc5@psu.edu)

At-sea experience has shown that target detection ranges are often less than predicted using current sonar system performance prediction methods. One possible reason for this disparity is that classical, sonar equationbased performance prediction models do not take into account the random and uncertain nature of signal propagation and scattering in a real ocean environment. Our goal is to develop a probabilistic sonar performance prediction methodology that can make use of limited knowledge of random or uncertain environment, target and sonar system parameters, but does not make unwarranted assumptions or rely upon unknown information. Beginning with models that describe how the ocean environment effects the received signal, the Maximum Entropy method (MEM) can be used to construct probability density functions (PDFs) for the parameters of those models. The benefit of the MEM is that it allows one to incorporate available knowledge about the parameters, but is maximally noncommittal about what is not known about the parameters. The methodology is presented and illustrated using a model for particular environmental effects on the structure of low-frequency signals propagating over long distances in the ocean. [Work supported by ONR Code 321US.] 


\section{0:15}

3aSP5. Stochastic filtering. David I. Havelock (Natl. Res. Council, 1200 Montreal Rd., Ottawa, ON, Canada K1A 0R6, david.havelock@nrc.ca)

Digital filters convolve a signal with an impulse response. This is equivalent to computing a weighted sum of delayed versions of the signal, with the signal versions indexed by their sample-delay and the weights given by the filter impulse response. This talk explores a different approach to filtering. Rather than computing a weighted sum of data values, the stochastic filter uses a sequence of random indices to select output data values from delayed versions of the signal. No summation is done. The probability distribution of the random indices is made identical to the normalized modulus of the impulse response of the corresponding conventional filter. For indices with negative weights, data values are negated. For a random index sequence $s(n)$ the stochastic filter output is simply $y(n)= \pm x(n-s(n))$. The output of such a stochastic filter is noisy but it is equal in the mean to the output of the corresponding conventional filter. If the sequence of random indices is identically and independently distributed then the noise is spectrally white. Such a filter can be useful for estimating spectral parameters with minimal computation. The principle is illustrated by an application to double-talk detection for telephones.

\section{0:30}

3aSP6. Autonomous signal separation microphone system based on the spatio-temporal gradient analysis. Kenbu Teramoto and Kosuke Tsuruta (Dept. of Mech. Eng., SAGA Univ., SAGA, 8408502, Japan)

This paper presents a novel autonomous directivity microphone system based on the newly proposed spatio-temporal blind source separation. Recently, the methods of blind source separation and multi-deconvolution of source signals have been proposed in various fields, especially, acoustic applications including the cocktail-party problem. The blind source separation principally uses no a priori knowledge about parameters of convolution, filtering and mixing. In the simplest case of the blind source separation problems, observed mixed signals are linear combinations of unknown mutually statistically independent, zero-mean source signals. The spatio-temporal blind signal separation algorithm utilizes the linearity among the four signals: (1) temporal gradient, (2) $x$; (3) $y$; and (4) $z$-directional spatial gradients of the sound-pressure, all of which are governed by the equation of advection. The proposed method, therefore, has an ability to simplify the convolution blind source separation problems into the instantaneous blind source separation over the spatio-temporal gradient space. The acoustical experiments are performed with the particle velocity microphone (Microflown) successfully instead of sound pressure microphones. Because, $x ; y$; and $z$-directional gradients of the sound pressure are equivalent the temporal derivatives of the corresponding directional particle velocities theoretically.

\section{0:45}

3aSP7. Bayesian sampling with applications to energy decay analysis in acoustically coupled spaces. Tomislav Jasa and Ning Xiang (School of Architecture and Dept. of Electrical, Computer, and Systems Eng., Rensselaer Polytechnic Inst., Troy, NY 12180)

Bayesian inference comprises of parameter estimation and model selection/comparison. A common approach to solving both of these problems has been to use statistical sampling techniques that are inherently non-Bayesian. This paper presents new Bayesian sampling method that solves both problems by changing the focus of Bayesian inference to the model selection problem first. In the papers [N. Xiang and P. M. Goggins, J. Acoust. Soc. Am. 110, 1415-1424 (2001); 113, 2685-2697 (2003)], the authors developed a model for the decay times and decay modes of acoustically coupled rooms in terms of measured Schroeder's decay functions. This paper shows how the Bayesian sampling method can be used to evaluate the "Bayesian evidence" term used in model selection as well as determining the decay times along with error estimates.
3aSP8. Adaptative Schur algorithm dedicated to underwater transient signal processing. Maciej Lopatka (Lab. d'Informatique Industrielle et Automatique, Univ. Paris XII, 61 av Gaulle, 94010 Créteil, France), Olivier Adam, Christophe Laplanche (Univ. Paris XII, France), Jan Zarzycki (Tech. Univ.of Wroclaw, Poland), and Jean-François Motsch (Univ. Paris XII, France)

The algorithm proposed by Lee and Morf [IEEE Transactions on Circuits and Systems 28(6) (1981)] which stems from the method defined by Schur [Operator Theory: Advances \& Application, Vol. 18 (Birk-Verlag, 1986)] has acquired a new significance [Zarzycki, Journal of Multidimensional Systems \& Signal Processing (Kluwer Academic, 2004)], due to its performances and particularly due to its applications in real time, made possible by the speed of processors available nowadays. Based on the innovations filter principle, Schur's proposal models the signal by calculating reflection coefficients, describing entirely the second-order signal. The reflection coefficients can be simply transformed to the AR coefficients, from which one derives the time-frequency representation. We compare performances of this approach with other time-frequency representations commonly used in the signal processing (spectrogram, AR, wavelet transform); we subsequently present the results obtained for transitory underwater acoustic signals, which our laboratory is investigating. The Lee and Morf algorithm offers an excellent tracking of the signal's characteristics and allows us to systematically detect transitory signals. This is particularly pertinent to segmentation problems relating to the application of underwater acoustics. The robustness of the Schur detector and a resolution of the Schur time-frequency representation support the resurgence of the Schur algorithm.

\section{1:15}

3aSP9. Laser Doppler signal for high amplitude sound wave. JeanChristophe Valiere, Solenn Moreau, Helene Bailliet (LEA-CNRS UMR 6609, Universite de Poitiers, France), Philippe Herzog (LMA-CNRS, Marseille, France), and Laurent Simon (LAUM-CNRS 6613, Universite du Maine, France)

Laser Doppler velocimetry (LDV) is now recognized as a very useful technique for estimating acoustic velocity with a good time resolution in many applications. Previous research showed that the most important parameter in LDV for acoustics is the particle displacement and particularly its magnitude compared with the size of the probe volume formed by the crossing of the laser beams. Specific techniques were developed to estimate the acoustic wave when the displacement is of the same order of magnitude as the probe diameter and when it is much smaller. In this study, we investigate situations where the displacement is much higher than the probe volume. The measuring process has been simulated numerically and it appears that the process leads to an under-estimation of the velocity around zero. This under-estimation is due to the processing of the laser Doppler signal in the case of high displacements. The associated signal is a non poissonnian randomly sampled signal to which classical processing methods are not adapted. It is compared to experimental signals obtained in the context of a study of non linear effects in an acoustic wave guide. To this end, different processing of both simulated and experimental signals are presented and compared.

\section{1:30}

3aSP10. Cross-frequency Doppler sensitive signal processing. Ronald A. Wagstaff (Natl. Ctr, for Physical Acoust., Univ. of Mississippi, Univ., MS 38677, rwagstaf@olemiss.edu)

When there is relative motion between an acoustic source and a receiver, a signal can be Doppler shifted in frequency and enter or leave the processing bins of the conventional signal processor. The amount of the shift is determined by the frequency and the rate of change in the distance between the source and the receiver. This frequency Doppler shifting can cause severe reductions in the processors performance. Special crossfrequency signal processing algorithms have recently been developed to mitigate the effects of Doppler. They do this by using calculation paths that cut across frequency bins in order to follow signals during frequency 
shifting. Cross-frequency spectral grams of a fast-flying sound source were compared to conventional grams, to evaluate the performance of this new signal processing method. The Doppler shifts in the data ranged up to 70 contiguous frequency bins. The resulting cross-frequency grams showed that three paths provided small to no improvement. Four paths showed improvements for either up-frequency or down-frequency shifting, but not for both. Two paths showed substantial improvement for both up-frequency and down-frequency shifting. The cross-frequency paths will be defined, and comparisons between conventional and cross-frequency grams will be presented. [Work supported by Miltec Corporation.]

\section{$11: 45$}

3aSP11. Eliminating clutter by coordinate zeroing. Harry DeFerrari (RSMAS-Univ. of Miami, 4900 Rickenbacker Cswy, Miami, FL 33149) and Andrew Rodgers (Woods Hole Group, East Falmouth, MA 02536)

Shallow water doppler sonar is limited by clutter that masks the presence of slow targets. Clutter arises from scattering from irregularities in the bottom or from fish schools. If the source and receiver are fixed in a bi-static sonar configuration, then the bottom scatterers (and sometimes the fish) have only a zero doppler component. Clutter arises from the doppler leakage resulting from sonar signals that are inherently short in time and therefore cannot resolve bottom reverberation in frequency. Here long continuous pulse compressions signals are considered (m-sequences). Ambiguity diagrams are compared with simple CW pulse and PCM pulses. The unique correlation properties of M-sequences lead to a signal sampling method that resolves sonar returns in complete ortho-normal $(\mathrm{CON})$ data sets. Zero doppler returns are identified as either direct arrivals or reverberation returns and then their particular coordinate can be set zero (coordinate zeroing) without affecting other data points. In this way, the zero-doppler bottom reverberation and all doppler leakage is eliminated. As an example, the return from a slow doppler target is imbedded in a very large number of reverberation returns that have higher signal level. As the zero doppler contributions are removed by coordinate zeroing the target becomes detectable.

WEDNESDAY MORNING, 18 MAY 2005

GEORGIA B, 8:15 A.M. TO 12:00 NOON

Session 3aUW

\title{
Underwater Acoustics and Acoustical Oceanography: Frequency Dependence of Sound Speed and Attenuation in Marine Sediments I
}

\author{
Gopu Potty, Cochair \\ Dept. of Ocean Engineering, Univ. of Rhode Island, Narragansett, RI 02882 \\ Peter H. Dahl, Cochair \\ Applied Physics Lab., Univ. of Washington, 1013 NE 40th St., Seattle, WA 98105-6698 \\ Chair's Introduction-8:15 \\ Invited Papers
}

8:20

3aUW1. Nonlinear frequency dependence of the effective seabottom acoustic attenuation from low-frequency field measurements in shallow water. Ji-Xun Zhou and Xue-Zhen Zhang (Georgia Inst. of Technol., Atlanta, GA 30332-0405 and Inst. of Acoust., Chinese Acad. of Sci., Beijing 100080, PROC)

Hamilton's seabed geo-acoustic model, which is widely accepted, predicts that the attenuation of sound in marine sediments increases linearly with frequency over the full frequency range of interest in ocean acoustics (a few hertz to megahertz). However, Biot-Stoll's physics-based seabed geo-acoustic model predicts that the bottom attenuation should exhibit non-linear frequency dependence, particularly in sandy bottoms. Since the publication of previous papers [Zhou, J. Acoust. Soc. Am. 78, 1003-1009 (1985); Kibblewhite, ibid. 86, 716-738 (1989)], more low-frequency field data, collected from different coastal zones around the world, have shown inconsistencies with the often-used linear frequency dependence. This paper attempts to support the non-linear frequency dependence of sea bottom attenuation through a review of shallow-water acoustic field measurements, with a special emphasis on the 50-1000 Hz frequency range. The relevant measurements include bottom reflection loss, sound transmission loss, dispersion analysis, vertical coherence of both propagation and reverberation, normal-mode spatial filtering, and optimum frequency and transition range of sound propagation. A non-linear frequency dependence of equivalent bottom acoustic attenuation, derived from these measurements, will be introduced. [Work supported by ONR and NNSF of China.]

\section{8:40}

3aUW2. Overview of SAX99 and SAX04 measurements of sediment sound speed and attenuation. Eric I. Thorsos, Kevin L. Williams, Darrell R. Jackson, and Dajun Tang (Appl. Phys. Lab., Univ. of Washington, 1013 NE 40th St., Seattle, WA 98105, eit@apl.washington.edu)

During SAX99 (for sediment acoustics experiment-1999) the sediment sound speed (125 Hz to $400 \mathrm{kHz})$ and attenuation $(2.5$ to $400 \mathrm{kHz}$ ) in sandy sediments were measured by a variety of techniques. The SAX99 site was $2 \mathrm{~km}$ from shore on the Florida Panhandle near Fort Walton Beach in water of 18-19 m depth. SAX04 was held in the fall of 2004 at a site close to the SAX99 site, about $1 \mathrm{~km}$ from shore in water of $17 \mathrm{~m}$ depth. The sediment sound speed and attenuation were again measured over a broad 
frequency range by multiple techniques, with even more attention paid to the low frequency band from $1-10 \mathrm{kHz}$. The results and corresponding uncertainties from SAX99 will be reviewed, and the consistency with Biot model predictions and alternative models (e.g., Buckingham's model) will be discussed. An overview will then be presented of the recently completed SAX04 measurement program on sediment sound speed and attenuation. [Work supported by ONR.]

\section{9:00}

3aUW3. Models for attenuation in marine sediments that incorporate structural relaxation processes. Allan D. Pierce, William M. Carey (Boston Univ., Boston, MA 02215, adp@bu.edu), and James F. Lynch (Woods Hole Oceanogr. Inst., Woods Hole, MA 02543)

Biot's model leads to an attenuation coefficient at low frequencies that is proportional to $\omega^{2}$, and such is consistent with physical models of viscous attenuation of fluid flows through narrow constrictions driven by pressure differences between larger fluid pockets within the granular configuration. Much data suggests, however, that the attenuation coefficient is linear in $\omega$ for some sediments and over a wide range of frequencies. A common model that predicts such a dependence stems from theoretical work by Stoll and Bryan [J. Acoust. Soc. Am. 47, 1440 (1970)], in which the elastic constants of the solid frame are taken to be complex numbers, with small constant imaginary parts. Such invariably leads to a linear $\omega$ dependence at sufficiently low frequencies and this conflicts with common intuitive notions. The present paper incorporates structural relaxation, with a generalization of the formulations of Hall [Phys. Rev. 73, 775 (1948)] and Nachman, Smith, and Waag [J. Acoust. Soc. Am. 88, 1584 (1990)]. The mathematical form and plausibility of such is established, and it is shown that the dependence is as $\omega^{2}$ at low frequencies, and that a likely realization is one where the dependence is linear in $\omega$ at intermediate frequency ranges.

9:20

3aUW4. Propagation and reverberation implications of seabed dispersion. Henrik Schmidt (MIT, 77 Massachusetts Ave., Cambridge, MA 02139)

The significance of the seabed geoacoustic properties to shallow water acoustic propagation is well established, and consequently the dispersion, or frequency dependence of the sound speed and the attenuation may be significant to the performance of broadband acoustic systems, whether for acoustic sensing or for communication. On the other hand, it is equally well established that the seabed significance is highly dependent on the system configuration, e.g., source and receiver depths, and the same will therefore obviously be the case for the dispersion. This paper will review the propagation effects of frequency dependent seabed geoacoustics, under characteristic seasonal environmental conditions, and typical system configurations. The associated implications to the acoustic system performance will be discussed in the context of theoretical performance bounds, such as the Cramer-Rao lower bounds, using established wave theory propagation models for range-independent and -dependent environments. Among the specific examples will be the effect of strong sediment dispersion in sandy sediments on buried target detection by low-frequency SAS systems operating in the 1-10 kHz regime, and broadband acoustic communication systems, including the effect of dispersion on seabed reverberation. [Work supported by ONR.]

\section{Contributed Papers}

9:40

3aUW5. Attenuation in the sediment layers in East China Sea. Gopu R. Potty and James H. Miller (Dept. of Ocean Eng., Univ. of Rhode Island, Narragansett, RI 02882, potty@oce.uri.edu)

Compressional wave attenuation in the frequency range 50 to $350 \mathrm{~Hz}$ is estimated using broadband data collected in the East China Sea. A time-frequency analysis provides amplitudes of individual modes which are used to estimate the modal attenuation coefficients. Different modes are sensitive to sediment properties at different depths and this modedepth sensitivity is explored in a sensitivity study. Based on this sensitivity study attenuation in different layers of the sediments will be estimated using different modes which are sensitive at these depths. This is a variation of previous studies where different frequencies have been used to invert for different sediment parameters using sub-space approaches. This approach focuses the inversion to various layers (provided by the geophysical surveys at the site) of the sediment and hence presents a tool to describe the depth variations in the sediment. The attenuation versus frequency behavior of the different sediment layers will be discussed. [Work supported by ONR.]
9:55-10:15 Break

\section{0:15}

3aUW6. Measuring dispersion and attenuation in sediment at low frequency. Steven A. Stotts, Robert A. Koch, and David P. Knobles (Appl. Res. Labs., Univ. of Texas, P.O. Box 8029, Austin, TX 78713-8029, stotts@arlut.utexas.edu)

Much work has been done at frequencies above $1 \mathrm{kHz}$ to measure sediment dispersion and attenuation and these results have been analyzed within the context of various models such as Biot-Stoll, Buckingham, and BICSQS. Some effort has been made to establish the frequency dependence of sediment attenuation below $1 \mathrm{kHz}$, but few results are available concerning dispersion in that frequency band. The present work examines a technique for measuring low frequency sediment dispersion and attenuation with a real dataset. Recently, data were collected off the coast of Florida using implosive broadband sources to perform geo-acoustic inversions. Previous inversion studies with this data ignored any dispersion effects. [Stotts et al, J. Acoust. Soc. Am. 115, 1078-1102 (2004).] The environment was shown to consist of thin, hard, porous material less than $1 \mathrm{~m}$ thick overlying sand sediment. A ray trace plus plane-wave reflection coefficient model was used for the analysis. Here, dispersion is repre- 
sented phenomenologically over a finite frequency band in the model by allowing both the sound speed and attenuation to be frequency dependent in both layers of a two-layer sediment plus substrate model.

\section{0:30}

3aUW7. Frequency dependence of posterior probability distributions for Biot parameters. Tracianne B. Neilsen, Marcia Isakson (Appl. Res. Labs., Univ. of Texas, P.O. Box 8029, Austin, TX 78713-8029, neilsen@arlut.utexas.edu), and Buye Xu (Brigham Young Univ., Provo, UT 84602)

The Biot-Stoll poro-elastic model for sediments is parameterized by 13 properties. Initial investigations indicate that the individual Biot parameters influence reflection loss differently depending on the frequency range of interest. A more extensive study of the frequency dependence of the parameter sensitivities is performed by computing posterior probability distributions (PPDs) [S. E. Dosso, J. Acoust. Soc. Am. 111, 129-142 (2002)] to determine which parameters most influence reflection loss for each frequency decade from $100 \mathrm{~Hz}$ to $1 \mathrm{MHz}$. The sampling for the PPDs is based on the least-squares error between simulated reflection coefficient data and values modeled with the reflection module of OASES. [H. Schmidt, "OASES Version 2.1 User Guide and Reference Manual," Department of Ocean Engineering, Massachusetts Institute of Technology, 1997.] The PPDs provide a quantitative value of the uncertainty in the optimum parameter estimates obtained by sampling or inversion techniques. A good value of the uncertainty is critical if one wants to use parameter estimates obtained with data from one frequency range to predict acoustic response for a different frequency range. [Work supported by the ONR.]

\section{0:45}

3aUW8. A hybrid model of sound propagation in unconsolidated sediments. Brian T. Hefner and Kevin L. Williams (Appl. Phys. Lab., Univ. of Washington, Seattle, WA 98105)

Efforts to model sound speed and attenuation in sandy sediments have centered on the use of theories for which either the relative motion of the pore fluid is the dominant attenuation mechanism, such as Biot theory, or the dominant loss mechanism is grain-to-grain friction. A recent model which attempts to incorporate grain-to-grain loss mechanisms into a model of sandy sediments was proposed by Buckingham. This model can fit the frequency dependence of the attenuation measured in ocean sediments and laboratory glass bead sediments, but it does not capture the sound speed dispersion as effectively as Biot theory. The relative success of each model suggests that both attenuation mechanisms may play important roles in sediment acoustics. In order to explore this possibility, a hybrid model has been developed which incorporates Buckingham's grain-to-grain shearing mechanisms into the frame moduli used in Biot theory. In the hybrid model, the grain-to-grain losses dominate at high and very low frequencies while pore fluid attenuation dominates at mid-frequencies where the sound speed dispersion is the most pronounced. As a consequence, the hybrid model is able to describe both the measured sound speed and attenuation in ocean and laboratory sediments. [Work supported by ONR.]

\section{1:00}

3aUW9. Attenuation in sand sediments due to scattering from force chains. Brian T. Hefner and Darrell R. Jackson (Appl. Phys. Lab., Univ. of Washington, Seattle, WA 98105)

The stresses in a granular media, such as an ocean sediment, are known to be heterogeneous and tend to form chains along which the stresses are particularly high. Because the elastic properties of a granular medium depend nonlinearly on the static forces between the grains, these force chains should induce inhomogeneities in the frame moduli. The correlation lengths of these inhomogeneities have been observed to be on the order of only a few grain diameters and as a consequence have not been thought to produce any significant scattering of acoustic energy. However, for high frequency sound in a saturated granular medium, these inhomogeneities should be on the order of the slow compressional wave. As a consequence, as the fast wave propagates through the sediment, it may scatter energy into the slow wave which is then rapidly attenuated. This scattering process may account for the deviation of the measured attenuation from the predictions of Biot theory that have been observed in ocean and laboratory sediments at high frequencies. We present a model of sound propagation through sand sediments that incorporates this loss mechanism and compare the results of this model to recent measurements. [Work supported by ONR.]

\section{1:15}

3aUW10. Sediment layer perturbation influences on the frequency dependence of effective bottom attenuation. James D. Nickila (Adv. Sonar Technol. Div. Naval Undersea Warfare Ctr., Newport, RI 02841), Kevin B. Smith (Naval Postgrad. School, Monterey, CA 93943), and Gopu Potty (Univ. of Rhode Island, Narragansett, RI 02882)

Over the past several years, concern has grown over the appropriateness of bottom attenuation models that assume a linear frequency dependence. Empirical analyses of experimental data have suggested power law dependence with frequency exponents as high as 1.7 and above, but with large variability between geographic regions. [Zhou et al., J. Acoust. Soc. Am. 82, 287-292 (1987).] The fundamental cause of this dependence is unknown. In this study, we investigate the effect of perturbations (both random and deterministic) in the sediment layer on the effective attenuation factor for a range of frequencies. A parabolic equation (PE) model is used to generate the acoustic field for a given environmental realization. The pressure field is decomposed using a normal mode expansion and an effective attenuation factor is calculated. Regression is performed on the resulting attenuation vs. frequency curve, with particular emphasis placed on testing for non-linearity.

\section{1:30}

3aUW11. Dependence of modal attenuation coefficient frequency variation on upper sediment attenuation. Wendy Saintval, William L. Siegmann (Dept. of Mathematical Sci., Rensselaer Polytech. Inst., 110 15th St., Troy, NY 12180), William M. Carey, Allan D. Pierce (Boston Univ., Boston, MA 02115), and James F. Lynch (Woods Hole Oceanogr., Inst., Woods Hole, MA 02543)

The range-averaged transmission loss increase in shallow water propagation depends critically on the intrinsic attenuation of the upper sediment. The attenuation coefficients of low-frequency $(<1 \mathrm{kHz})$ propagating modes determine the frequency dependence. Ingenito [J. Acoust. Soc. Am. 53, 858-863 (1973)] showed with measurements and theory that while individual mode attenuation coefficients decrease with frequency $f$, the sediment attenuation coefficient increases proportional to $f^{1.75}$. When results from many other shallow-water transmission experiments (broadband and narrowband) over sandy-silty sediments are compared to numerical calculations, it is found that a nonlinear-frequency dependent attenuation is required with an exponent between 1.5 and 2. The question considered here is how the intrinsic upper-sediment attenuation produces such behavior. A recent simplification of the Biot model [A. D. Pierce et al., J. Acoust. Soc. Am. 114, 2345 (2003)] has a power-law exponent of two. With this frequency-dependent bottom attenuation, a two-layer Pekeris waveguide yields modal attenuation coefficients that decrease with frequency as observed by Ingenito. However, a depth-dependent attenuation profile or a third near-surface layer with requisite properties can reverse this behavior. This suggests why higher-frequency numerical computations may require nonlinear frequency-dependent sediment profiles to calculate sound transmission accurately. [Work partially supported by ONR.]

\section{$11: 45$}

3aUW12. Frequency dependence of the frame bulk moduli of granular marine sediments. Masao Kimura (Dept. of Geo-Environ. Technol., Tokai Univ., 3-20-1 Shimizu-Orido, Shizuoka, Shizuoka 424-8610, Japan, mkimura@scc.u-tokai.ac.jp)

The frame bulk modulus is important in analyzing the acoustic wave propagation in porous granular marine sediments. As the value of the frame bulk modulus, notable researchers in the community used about $10^{8} P_{a}$ (constant value). However, the measured frequency characteristics 
of the longitudinal wave velocity are not consistent with the calculated characteristics using the constant frame bulk modulus using the Biot-Stoll model. It was reported that the frame bulk moduli in water-saturated glass beads and beach sands are about $10^{9} P_{a}$ at the frequency of $500 \mathrm{kHz}$, and are about ten times greater than that of air-saturated samples. It is also seen that the frame bulk moduli have dependence on the grain size. Moreover, these phenomena can be explained using the gap stiffness model [M.
Kimura, J. Acoust. Soc. Am. 116, 2529 (2004)]. The grain size dependence of the frame bulk modulus suggests that there is the frequency dependence of the modulus. In this study, the measured results of the frequency characteristics of the longitudinal wave velocity in water-saturated glass beads and beach sand are compared with the calculated results using the BiotStoll model with the frequency dependent frame bulk modulus, derived using the gap stiffness model.

\title{
Meeting of Accredited Standards Committee (ASC) S2 Mechanical Vibration and Shock
}

to be held jointly with the

\author{
ANSI-Accredited U.S. Technical Advisory Group (TAG) Meetings for: \\ ISO/TC 108 Mechanical Vibration and Shock
}

ISO/TC 108/SC 2 Measurement and evaluation of mechanical vibration and shock as applied to machines, vehicles and structures

ISO/TC 108/SC 3 Use and calibration of vibration and shock measuring instruments

ISO/TC 108/SC 4 Human exposure to mechanical vibration and shock

ISO/TC 108/SC 5 Condition monitoring and diagnostics of machines

and

ISO/TC 108/SC 6 Vibration and shock generating systems

\author{
R. J. Peppin, Chair S2 \\ 5012 Macon Road, Rockville, MD 20852
}

D. J. Evans, Vice Chair S2 and Chair of the U.S. Technical Advisory Group (TAG) for ISO/TC 108

Mechanical Vibration and Shock

and Chair of the U.S. Technical Advisory Group (TAG) for ISO/TC 108/SC 3

Use and calibration of vibration and shock measuring devices

National Institute of Standards and Technology (NIST), 100 Bureau Drive, Stop 8221, Gaithersburg, MD 20899-8221

A. F. Kilcullen, Co-Chair of the U.S. Technical Advisory Group (TAG) for ISO/TC 108/SC 2

Measurement and evaluation of mechanical vibration and shock as applied to machines, vehicles and structures

5012 Woods Road, Hedgesville, WV 25427

R. Taddeo, Co-Chair of the U.S. Technical Advisory Group (TAG) for ISO/TC 108/SC 2

Measurement and evaluation of mechanical vibration and shock

as applied to machines, vehicles and structures

Naval Sea Systems Command 05T, 1339 Patterson Ave. SE, Stop 8800, Washington Navy Yard, Washington DC 20376-8800

D. D. Reynolds, Chair, U.S. Technical Advisory Group (TAG) for ISO/TC 108/SC 4

Human exposure to mechanical vibration and shock

3939 Briar Crest Court, Las Vegas, NV 89120

D. J. Vendittis, Chair of the U.S. Technical Advisory Group (TAG) for ISO/TC 108/SC 5

Conditon monitoring and diagnostics of machines

701 Northeast Harbour Terrace, Boca Raton, FL 33431

G. Booth, Chair of the U.S. Technical Advisory Group (TAG) for ISO/TC 108/SC 6

Vibration and shock generating systems

44 Bristol Street, Branford, CT 06405-4842

\begin{abstract}
Accredited Standards Committee S2 on Mechanical Vibration and Shock. Working group chairs will report on the status of various shock and vibration standards currently under development. Consideration will be given to new standards that might be needed over the next few years. There will be a report on the interface of S2 activities with those of ISO/TC 108 and its subcommittees including plans for future meetings of ISO/TC 108 and/or its Subcommittees. The Technical Advisory Groups for ISO/TC 108 and and its Subcommittees consists of members of S2 and other persons not necessarily members of those Committees. Open discussion of committee reports is encouraged.
\end{abstract}


Scope of S2: Standards, specifications, methods of measurement and test, and terminology in the field of mechanical vibration and shock, and condition monitoring and diagnostics of machines, including the affects of exposure to mechanical vibration and shock on humans, including those aspects which pertain to biological safety, tolerance and comfort.

WEDNESDAY MORNING, 18 MAY 2005

STANLEY ROOM, 10:30 A.M. TO 12:00 NOON

\title{
Meeting of Accredited Standards Committee (ASC) S3 Bioacoustics
}

\author{
R. F. Burkard, Chair S3 \\ Hearing Research Laboratory, State University of New York at Buffalo, 215 Parker Hall, Buffalo, NY 14214 \\ C. A. Champlin, Vice Chair S3 \\ Department of Communication Sciences \& Disorders, University of Texas, CMA 2-200, Austin, TX 78712
}

\begin{abstract}
Accredited Standards Committee S3 on Bioacoustics. Working group chairs will report on the status of standards under development. Consideration will be given to new standards that might be needed over the next few years. Open discussion of committee reports is encouraged.
\end{abstract}

People interested in attending the meeting of the TAGs for ISO/TC 43 Acoustics and IEC/TC 29 Electroacoustics, take note - those meetings will be held in conjunction with the Standards Plenary meeting at 10:30 a.m. on Tuesday, 17 May 2005.

Scope of S3: Standards, specifications, methods of measurement and test, and terminology in the fields of psychological and physiological acoustics, including aspects of general acoustics, which pertain to biological safety, tolerance, and comfort.

WEDNESDAY AFTERNOON, 18 MAY 2005

REGENCY E, 1:00 TO 3:00 P.M.

\section{Session 3pAA}

\section{Architectural Acoustics: Scattering in Architectural Acoustics}

\author{
Kerrie G. Standlee, Chair \\ 4900 SW Griffith Dr., Suite 216, Beaverton, OR 97005
}

Contributed Papers

1:00

3pAA1. Here and there, near and far: How proximity and separation affect scattering in concert halls. James B. Lee (6016 S. E. Mitchell, Portland, OR 97206)

Classical theory of scattering deals with plane waves, waves which are so far from their source that they form straight fronts of sound, with pressure amplitude and particle velocity exactly in phase. If such waves are much larger than objects they encounter inverse fourth power (Rayleigh) scattering obtains; if the waves are about the same size as the objects resonant (Mie) scattering occurs; if waves are much shorter than the objects specular (Ufimtsev) scattering is the rule. These all affect sound in the far field. But if sources are closer than a wavelength to objects their waves encounter the plane approximation is not valid; pressure amplitude and particle velocity are not in phase, so resonance-like phenomena occur. These occur on stages of concert halls: bass instruments producing waves $2 \mathrm{~m}$ or longer always are close to the floor; some, like tympani and viols, can be close to vertical surfaces too. This sort of scattering enhances fundamentals of notes with respect to the overtones, strongly affecting the timbre of such instruments.
1:15

3pAA2. Diagnosing scattering with the Wigner distribution. James B. Lee (6016 S. E. Mitchell, Portland, OR 97206)

The Wigner distribution resolves the power of a signal in both the time domain and the frequency domain. It also is a complete representation because it displays the relative phases of a signal's components as crossproducts between them. This means that if one records the time-series of an impulse response in a room and computes a Wigner distribution from it, the kind of scattering, predominantly resonant or predominantly specular, is readily apparent, because the phase response of each is radically different. Specular scattering preserves the relative phases among components comprising the initial impulse and so preserves the impulse itself; resonant scattering randomizes the phases among components and so converts an impulse into a wide band of frequencies spread smoothly in time. Thus specular scattering tends to promote echoes and resonant scattering tends to promote diffusion. Graphic examples of each are given in the literature. 
3pAA3. The influence of surface diffusivity on reverberation time prediction. Yan Zhang and Godfried Augenbroe (College of Architecture, Georgia Inst. of Technol., Atlanta, GA 30332)

It has been reported in the literature that the reverberation time tends to be overestimated if the ray-tracing simulation does not consider surface scattering effects. However no simple, quantitative method is available to estimate the effect of surface diffusivity on reverberation time. This article provides such a model to establish the relation between reverberation time and a surface diffusivity factor. It has also been observed that the influence of scattering reflections increases when the material is less uniformly distributed. Therefore the authors studied the current prediction method with non-uniformly distributed material and observed the following: Kutruff equation based on the diffusive surface assumptions yields shorter reverberation times than Eyrings formula, while another equation containing reflective assumptions yields longer RT than Eyrings formula. It is demonstrated that the difference can reach up to $50 \%$. Therefore the authors propose a linear combination of these two equations, with the proportion to each end determined by the surface diffusivity, assessed against Hann and Fricks method. A comparison between Boston Symphony Hall and New York Avery Fisher Hall is performed to demonstrate the significance of the surface diffusivity, and a Monte-Carlo simulation is included to support the result even under parameter uncertainties.

\section{$1: 45$}

3pAA4. Aurally-adequate time-frequency analysis for scattered sound in auditoria. Molly K. Norris, Ning Xiang, and Mendel Kleiner (Dept. of Architectural Acoust., Rensselaer Polytechnic Inst., 110 8th St., Troy, NY 12180)

The goal of this work was to apply an aurally-adequate time-frequency analysis technique to the analysis of sound scattering effects in auditoria. Time-frequency representations were developed as a motivated effort that takes into account binaural hearing, with a specific implementation of interaural cross-correlation process. A model of the human auditory system was implemented in the MATLAB platform based on two previous models [A. Härmä and K. Palomäki, HUTear, Espoo, Finland; and M. A. Akeroyd, A. Binaural Cross-correlogram Toolbox for MATLAB (2001), University of Sussex, Brighton]. These stages include proper frequency selectivity, the conversion of the mechanical motion of the basilar membrane to neural impulses, and binaural hearing effects. The model was then used in the analysis of room impulse responses with varying scattering characteristics. This paper discusses the analysis results using simulated and measured room impulse responses. [Work supported by the Frank H. and Eva B. Buck Foundation.]

\section{2:00}

3pAA5. Characteristics of scattered sound from overhead reflector arrays: Measurements at 1:8 and full scale. Timothy Gulsrud (Kirkegaard Assoc., 954 Pearl St., Boulder, CO 80302, tgulsrud@ kirkegaard.com)

Measurements of sound scattering from reflector arrays have been carried out both at 1:8 scale in a controlled environment and at full scale in a concert hall. The results indicate wave interference between reflections from adjacent panels, resulting in an audible comb filter-like effect. Implications for the appropriate degree of overlap in coverage of adjacent panels will be discussed.
3pAA6. Effect of incidence angle and panel size on the phase of scattered energy from panel arrays. Jonathan Rathsam, Lily M. Wang (Architectural Eng. Program, Univ. of Nebraska Lincoln, 247 PKI, 1110 S. 67th St., Omaha, NE 68182-0681, jrathsam@mail.unomaha.edu), and Rendell R. Torres (Rensselaer Polytechnic Inst., Troy, NY 12180-3590)

This investigation focuses on factors that influence scattering from different ceiling panel arrays (e.g., incidence angle, and array size and density) by examining the scattered phase. A previous study by the third author [Torres and Vorländer, Acta Acust. (in press)] measured impulse responses using the maximum length sequence method at various receiver positions from scale model panel arrays of different sizes and densities. One interesting conclusion was that for multi-element arrays with panel dimensions and separation on the order of a wavelength, the reflected amplitude decreased, apparently due to destructive interference from interpanel scattering. Analysis focused on the magnitude of the frequency response but the author noted that the phase curves could also contain information about scattered energy and the hypothesized inter-panel scattering. What the phase curves reveal is the subject of this study. It is expected that noticeable deviations from smoothness observed in the phase curves indicate non-specular scattering from the panels. The frequencies at which these deviations occur are examined to give insight into how the geometrical conditions of the panel array affect scattering.

$2: 30$

3pAA7. A comparison of partially specular radiosity and ray tracing for room acoustics modeling. C. Walter BeamerIV (Dept. of Civil, Env., and Arch. Eng., Univ. of Colorado, Boulder, CO 80309) and Ralph T. Muehleisen (Illinois Inst. of Technol., Chicago, IL 60616)

Partially specular (PS) radiosity is an extended form of the general radiosity method. Acoustic radiosity is a form of bulk transfer of radiant acoustic energy. This bulk transfer is accomplished through a system of energy balance equations that relate the bulk energy transfer of each surface in the system to all other surfaces in the system. Until now acoustic radiosity has been limited to modeling only diffuse surface reflection. The new PS acoustic radiosity method can model all real surface types, diffuse, specular and everything in between. PS acoustic radiosity also models all real source types and distributions, not just point sources. The results of the PS acoustic radiosity method are compared to those of well known ray tracing programs. [Work supported by NSF.]

\section{2:45}

3pAA8. A new scattering method that combines roughness and diffraction effects. Claus Lynge Christensen (Odeon A/S c/o Acoust. Technol., rsted-DTU, Bldg. 352, DK-2800 Lyngby, Denmark)

Most of today's room acoustics programs make use of scattering coefficients which are used in order to describe surface scattering (roughness of material) and scattering of reflected sound caused by limited surface size (diffraction). A method which combines scattering caused by diffraction due to typical surface dimension, angle of incidence, and incident path length with surface scattering is presented. Each of the two scattering effects is modeled as frequency dependent functions. The benefits are two-fold: (a) Separating the user specified surface scattering coefficient from the room geometry, makes it easier for the user to make good guesstimates of the coefficients which will be in better agreement with the ones which can be measured. In many cases a scattering coefficient of say $1 \%$ for all surfaces may be sufficient. (b) Scattering due to diffraction is distance and angle dependent and as such it is not known before the actual raytracing or image source detection takes place. An example of this is that a desktop will provide a strong specular component to its user whereas it will provide scattered sound at far distances. 


\title{
Session 3pAO
}

\section{Acoustical Oceanography: Acoustical Oceanography Prize Lecture}

\author{
N. Ross Chapman, Chair \\ Univ. of Victoria, School of Earth and Ocean Sciences, Victoria, BC V8W 3P6, Canada
}

Chair's Introduction-1:30

Invited Paper

$1: 35$

3pAO1. Acoustic explorations of the upper ocean boundary layer. Svein Vagle (OSAP, Inst. of Ocean Sci., P.O. Box 6000, Sidney, BC, Canada V8L 4B2, vagles@pac.dfo-mpo.gc.ca)

The upper ocean boundary layer is an important but difficult to probe part of the ocean. A better understanding of small scale processes at the air-sea interface, including the vertical transfer of gases, heat, mass and momentum, are crucial to improving our understanding of the coupling between atmosphere and ocean. Also, this part of the ocean contains a significant part of the total biomass at all trophic levels and is therefore of great interest to researchers in a range of different fields. Innovative measurement plays a critical role in developing our understanding of the processes involved in the boundary layer, and the availability of low-cost, compact, digital signal processors and sonar technology in self-contained and cabled configurations has led to a number of exciting developments. This talk summarizes some recent explorations of this dynamic boundary layer using both active and passive acoustics. The resonant behavior of upper ocean bubbles combined with single and multi-frequency broad band active and passive devices are now giving us invaluable information on air-sea gas transfer, estimation of biological production, marine mammal behavior, wind speed and precipitation, surface and internal waves, turbulence, and acoustic communication in the surf zone.

\section{Session 3pID}

\section{Interdisciplinary: Hot Topics in Acoustics}

\author{
Beverly A. Wright, Chair \\ Dept. of Communication Science and Disorders, Northwestern Univ., 2240 Campus Dr., Evanston, IL 60208
}

Chair's Introduction-1:30

Invited Papers

$1: 35$

3pID1. The Student Council. David T. Bradley (Univ. of Nebraska-Lincoln, 1110 S. 67th St., PKI 245 Omaha, NE 68182-0681, dbradley@mail.unomaha.edu)

Throughout its history, the Society has been a strong advocate for students exploring the world of acoustics. However, only recently was that support institutionalized and the responsibility placed in the hands of the students themselves. In the spring of 2000 , a nascent body was formed to address the needs and concerns of Student Members of the ASA. The Vancouver meeting marks the 5th anniversary of this group, now formally known as the Student Council. The Council has evolved markedly in the past several years. The Council is currently composed of one student representative from each of the 13 technical committees and one Student Chapter Liaison. Recent student focused activities include the creation of a state-of-the-art website, the production of the first in a series of fellowship/grant workshops, and the presentation of a new award honoring the efforts of mentors in the Society. The Councils main focus is disseminating information of concern to students, advocating participation of students in Society activities and meetings, and promoting Society membership benefits to potential student members. Current and future programming and opportunities available to students, and those who work with students, will be presented and discussed. 
3pID2. The ASA Regional Chapters program. Elizabeth McLaughlin (Chair of Committee on Regional Chapters) and Juan Arvelo (Co-Chair of Committee on Regional Chapters, Johns Hopkins Univ., Appl. Phys. Lab., 11100 Johns Hopkins Rd., Laurel, MD 20723-6099)

Are you involved in a Regional Chapter? The Regional Chapters Program certainly embraces the intent of our Society. "The ASA was founded... to increase and diffuse the knowledge of acoustics and promote its practical applications. Any person ... interested in acoustics is eligible for membership." The history and the activities of each Chapter are unique. There are currently twenty active chapters uniquely positioned to promote acoustics through outreach and involvement with the public. There have been several new developments in the Regional Chapters Program, the most exciting being the incorporation of Student Chapters! Our first, the Nebraska Student Chapter, was approved at the 75th Meeting of the ASA in NYC. Several more are on their way! Existing Chapters are revitalizing! The Washington DC Chapter has recently found new enthusiasm, re-establishing a robust program. A new student scholarship has been organized thanks to the generosity of Larry and Julia Royster. Another recent enhancement is the expansion of the Regional Chapters Website. There one can find useful materials including an updated Chapter Start-up Kit. Involvement in a chapter is a great way to give back to the ASA, to learn, promote acoustics, to socialize, and to involve new persons in our exciting field!

2:15

3pID3. Imaging for understanding speech communication: Advances and challenges. Shrikanth Narayanan (Signal and Image Processing Inst., Univ. of Southern California, Viterbi School of Eng., Los Angeles, CA 90089-2564)

Research in speech communication has relied on a variety of instrumentation methods to illuminate details of speech production and perception. One longstanding challenge has been the ability to examine real-time changes in the shaping of the vocal tract; a goal that has been furthered by imaging techniques such as ultrasound, movement tracking, and magnetic resonance imaging. The spatial and temporal resolution afforded by these techniques, however, has limited the scope of the investigations that could be carried out. In this talk, we focus on some recent advances in magnetic resonance imaging that allow us to perform near real-time investigations on the dynamics of vocal tract shaping during speech. Examples include Demolin et al. (2000) (4-5 images/second, ultra-fast turbo spin echo) and Mady et al. $(2001,2002)$ (8 images/second, T1 fast gradient echo). A recent study by Narayanan et al. (2004) that used a spiral readout scheme to accelerate image acquisition has allowed for image reconstruction rates of 24 images/second. While these developments offer exciting prospects, a number of challenges lie ahead, including: (1) improving image acquisition protocols, hardware for enhancing signal-to-noise ratio, and optimizing spatial sampling; (2) acquiring quality synchronized audio; and (3) analyzing and modeling image data including cross-modality registration. [Work supported by NIH and NSF.]

A non-inclusive sampling of recent advances in the application of physical acoustics to condensed matter physics is presented. Work by Ogi et al. on resonant ultrasound microscopy to map nano-scale variations in elastic moduli provides a new twist to the use of resonances. Laser-ultrasound studies by Wright and Matsuda on photoacoustic effects in ultra-thin metallic and semiconductor films leads to new meso-scale ultrasound studies, while Isaak and Ohno push the symmetry limits and temperature range of resonant ultrasound modulus studies. Migliori and Ledbetter extract the final pieces of an unusually-well-characterized charge-density-wave phase transition and examine very odd elastic behavior in materials with negative thermal expansion, while Pantea demonstrates an all-digital pulse echo system for high pressure work. Souslov probes hidden order with pulse-echo studies in pulsed magnetic fields. [Work supported by the National Science Foundation, the Department of Energy and the State of Florida.]

3pID5. Speech perception in the presence of other sounds. C. J. Darwin (Dept. of Psychol., Univ. of Sussex, Brighton BN1 9QG, UK)

The human listener's remarkable ability to recognize speech when it is mixed with other sounds presents a challenge both to models of speech perception and to approaches to speech recognition. This talk will review some of the work on how human listeners can perceive speech in sound mixtures and will try to indicate areas that might be particularly fruitful for future research. 
Session 3pSA

\title{
Structural Acoustics and Vibration: Approximation Techniques for Fluid Loading Effects II
}

\author{
Joel M. Garrelick, Cochair \\ Cambridge Acoustical Associates/ETC, 84 Sherman St., Cambridge, MA 02140 \\ David Feit, Cochair \\ Applied Physical Sciences, Inc., 2 State St., New London, CT 06320
}

Invited Papers

$1: 15$

3pSA1. Doubly asymptotic approximations for transient and time-harmonic acoustics. Thomas L. Geers (Dept. of Mech. Eng., Univ. of Colorado, Boulder, CO 80309-0427)

The development and application of doubly asymptotic approximations (DAAs) in computational structural acoustics are reviewed. Transient radiation/scattering is emphasized, but application to time-harmonic radiation/scattering is also addressed. DAAs formulated for elastodynamic, poroelastic, and electromagnetic scattering are described that illustrate the breadth of application. The advantages and limitations of DAAs are described, and their relation to retarded-potential, similarity-based, and infinite-element methods are discussed.

$1: 45$

3pSA2. Extension of infinite elements to explicit dynamic and eigenanalysis of submerged structures. Jeffrey Cipolla (ABAQUS, Inc., 166 Valley St., Providence, RI 02909-2499)

\begin{abstract}
Infinite elements are distinct from alternative methods for exterior problems in that, like a finite element, they describe a small sub-region of the problem domain, and use locally supported shape functions to derive a method of weighted residuals statement thereon. Until recently, transient analyses using explicit time-integration methods, and eigenanalysis, were not possible using infinite elements. A formulation of acoustic infinite element compatible with implicit dynamics, time-harmonic acoustics, far-field extrapolation, explicit dynamics, and eigenanalysis is described. First steps in the development are the adoption of a basis corresponding to spherical radiating harmonics, the Bettess geometric map, and the Astley-Leis weighted residual formulation. A modified means to compute the element integrals, and a modification of the spherical harmonic basis, improve numerical conditioning of the element and stability of the formulation. The trivial frequency dependence of the Astley-Leis formulation, critical for its application to transient problems, also enables a formulation for eigenanalysis, which will be discussed. Finally, a splitting of the element operator into tangential mass- and stiffness-like terms enables a partial lumping of the element, and compatibility with explicit time integration schemes. Importantly, this splitting preserves the essential radiation mode accuracy of the formulation. Some computational examples are shown.
\end{abstract}

3pSA3. Numerical study of fluid loading approximations for submerged spherical shells using finite, infinite and boundary element methods. Rui M. Botelho (Electric Boat Corp., Dept. 462-M.S. J129-2, 75 Eastern Point Rd., Groton, CT 06340-4989, rbotelho@ebmail.gdeb.com), Michael J. Butler (Electric Boat Corp., Groton, CT 06340-4989), and John F. Waters (Electric Boat Corp., Groton, CT 06340-4989)

Numerical studies were conducted to compare acoustic fluid loading approximations for submerged spherical shells. Rigid and empty elastic shells submerged in a heavy acoustic fluid and subjected to harmonic excitation were considered. Solutions using boundary elements, infinite elements, and analytical approximations were compared, with special attention to modeling the near-field fluid beneath the infinite elements. In this case, the near-field fluid is typically modeled using fluid finite elements with frequency dependent mesh refinement and radial extent. The spherical shell is modeled in the same manner using shell finite elements for both boundary and infinite element solutions in order to provide a direct comparison of the fluid loading approximations. Convergence studies involving the near-field fluid were performed with emphasis on the effects of meshing parameters, including refinement, mesh grading, and polynomial order of the radial basis functions for $p$-version fluid finite elements. Results of interest include comparisons of the submerged structural response, focusing on the reactive and resistance parts of the fluid impedance. 
Session 3pUW

\title{
Underwater Acoustics and Acoustical Oceanography: Frequency Dependence of Sound Speed and Attenuation in Marine Sediments II
}

\author{
Gopu Potty, Cochair \\ Dept. of Ocean Engineering, Univ. of Rhode Island, Narragansett, RI 02882 \\ Peter H. Dahl, Cochair \\ Applied Physics Lab., Univ. of Washington, 1013 NE 40th St., Seattle, WA 98105-6698
}

Contributed Papers

$1: 30$

3pUW1. Frequency dependence of elliptical particle motion of acoustic waves transmitted into the seabed from a point source in water. David M. F. Chapman, Paul C. Hines, and John C. Osler (Defence Res. and Development Canada Atlantic, P.O. Box 1012, Dartmouth, NS, Canada B2Y 3Z7, dave.chapman@drdc-rddc.gc.ca)

When perpendicular components of the particle velocity of a continuous acoustic wave have unequal amplitude and phase, the displacement traces an elliptical path. This is caused by inhomogeneous waves and/or losses in the medium. For spherical waves radiating from a point source in water and transmitted into the seabed, the orientation of the elliptical orbit varies with receiver location and frequency, and the ellipse major axis is not always parallel to the direction of propagation suggested by Snell's law. If not accounted for, this could bias estimates of sediment sound speed derived from particle velocity measurements. This presentation describes a simple orbit model based on numerical evaluation of integrals for the transmitted field over the entire wavenumber range. The model provides rapid simulation of results for different experimental geometries and source frequencies. The model is validated using an established full-field model and compared with experimental data. [Work supported in part by ONR Code 32.]

\section{$1: 45$}

3pUW2. Acoustic velocity measurements in seafloor sands at frequencies from 1 to $400 \mathrm{kHz}$. Michael A. Zimmer, L. Dale Bibee, and Michael D. Richardson (Naval Res. Lab., 1005 Balch Blvd., Stennis Space Center, MS 39529-5004)

We measured the acoustic velocity and attenuation at frequencies from $1 \mathrm{kHz}$ to $400 \mathrm{kHz}$ in shallow seafloor sands off Fort Walton Beach, FL, USA. We used three separate systems with overlapping frequency ranges in order to cover this entire frequency range. For frequencies from 1 to 20 $\mathrm{kHz}$, we implanted a seafloor array of 35 hydrophones and 5 threecomponent accelerometers at depths from 0 to $1 \mathrm{~m}$ over a $4 \mathrm{~m}$ by $4 \mathrm{~m}$ area, and recorded signals from two acoustic sources positioned at offsets from $1 \mathrm{~m}$ to $20 \mathrm{~m}$. Measurements from 15 to $120 \mathrm{kHz}$ were made at $30-\mathrm{cm}$ sediment depths with the In Situ Sediment Acoustic Measurement System (ISSAMS), which consists of a linear array of 4 piezoelectric probes; the outer probes transmit a single-frequency burst while the inner probes act as receivers. Additionally, velocity measurements were made on divercollected cores (5-20 cm sediment depths) at frequencies from 50 to 400 $\mathrm{kHz}$. We present comparisons of the measured frequency dependence of the acoustic velocity and attenuation to theoretical predictions from various constitutive and wave propagation models, parameterized with inputs from detailed characterizations of diver-collected cores.
2:00

3pUW3. Inverting for sandy sediment sound speed in very shallow water using boat noise. Dajun Tang (Appl. Phys. Lab, Univ. of Washington, 1013 NE 40th St., Seattle, WA 98105)

Small boat propeller noise was recorded on a single hydrophone in very shallow water for the purpose of estimating sediment sound speed. The experiment was conducted near shore in water depth between 2 and $10 \mathrm{~m}$. The sediment consisted of uniform sand. The lone hydrophone was moored $2 \mathrm{~m}$ above the bottom at $6 \mathrm{~m}$ depth. A small boat traveling at constant speed was used as the sound source, and ran both parallel and perpendicular to shore. Thus, both the range independent waveguide case and wedge shaped waveguide case could be investigated. The source tracks were recorded by using a GPS recorder on the boat. Water depth in the entire area was measured, as was the sound speed profile at the receiver. The processed date sets resulted in interference patterns in rangefrequency plots. Aided by numerical simulations, sediment sound speed can be estimated over the frequency range of 500-4500 Hz. [Work supported by the U.S. Office of Naval Research.]

\section{2:15}

3pUW4. Effect of frequency dependence of complex sound speed on impulse response in littoral seas. David Knobles (Appl. Res. Labs., Univ. of Texas, P.O. Box 8029, Austin, TX 78713-8029)

For certain classes of sediments common to littoral ocean environments, there exists theoretical reasons and experimental evidence that the real part of the sound speed is frequency dependent. Further it has been observed for more than 30 years that to model transmission loss data below $1 \mathrm{kHz}$ for such sediments, it is often required that the attenuation possess a non-linear frequency dependence. However, analyses of broadband propagation below $2 \mathrm{kHz}$ in littoral waveguides commonly assume that sound speeds in marine sediments are frequency independent and that the attenuation depends linearly on frequency. It is natural to explore how the neglect of the frequency dependence of the complex sound speed affects the predictability of broadband propagation and under what conditions can inversion methods estimate the functional form of the frequency dependence. In this study simulated impulse responses for typical littoral waveguides over the $10-2000 \mathrm{~Hz}$ band are generated assuming a BiotStoll formulation. The effects of the frequency dependence of the complex sound speed on the impulse response are examined within the context of how does one form a valid inverse problem that would allow for the frequency dependence of the complex sound speed to be uniquely determined. [Work supported by ONR.] 
3pUW5. Longitudinal wave dispersion and attenuation in a two phase granular material. Hasson Tavossi (Dept. of Physical and Environ. Sci., Mesa State College, 1100 North Ave., Grand Junction, CO 81501)

Longitudinal elastic wave-velocity and attenuation in a two phase granular material are investigated, in the cases of a random arrangement of grains in contact with each other, surrounded by water or air. The velocity dispersion and attenuation of a longitudinal wave are measured experimentally, at low and high frequencies and are analyzed in light of a modi- tenuation are expressed as a function of frequency, grain size, porosity, viscosity of the fluid phase, as well as elastic moduli and densities of the solid and fluid phases. It is shown that longitudinal wave velocity in such a material decreases with grain size and frequency at high frequencies. Results obtained by increasing the grain size resemble those measured by increasing the frequency. Wave attenuation is expressed as a function of frequency, porosity, grain size and fluid viscosity. Theoretical results are compared with the experimental findings on the wave velocity and attenuation.

WEDNESDAY AFTERNOON, 18 MAY 2005

REGENCY A, B, C, 3:30 TO 5:30 P.M.

Plenary Session, Business Meeting and Awards Ceremony

\author{
William A. Kuperman, President \\ Acoustical Society of America \\ Stan E. Dosso, President \\ Canadian Acoustical Association
}

Welcome Remarks

\title{
Annual Business Meeting of the Acoustical Society of America
}

\section{Presentation of Certificates to New Fellows}

\author{
Catherine T. Best \\ Bennett M. Brooks \\ Michael G. Brown \\ Charles F. Gaumond \\ Murray R. Hodgson
}

Presentation of Acoustical Society Awards

Medwin Prize in Acoustical Oceanography to Svein Vagle

Silver Medal in Psychological and Physiological Acoustics to H. Steven Colburn

R. Bruce Lindsay Award to Lily M. Wang

Helmholtz-Rayleigh Interdisciplinary Silver Medal to Gilles A. Daigle

Gold Medal to Allan D. Pierce 


\title{
Session 3eAA
}

\section{Architectural Acoustics, Education in Acoustics, Noise and Psychological and Physiological Acoustics: Topical Meeting on Classroom Acoustics-Public Forum}

\author{
Murray R. Hodgson, Cochair \\ Univ. of British Columbia, School of Occupational and Environmental Hygiene, 2206 East Mall, \\ Vancouver, BC V6T 1Z3, Canada \\ Maureen MacDonald, Cochair \\ Elementary School Teacher, Vancouver, BC, Canada
}

Chair's Introduction-6:30

6:35

3eAA1. The need for good acoustic design of schools. Bridget Shield (Dept. of Eng. Systems, Faculty of Eng. Sci. and Built Environment, London South Bank Univ., London SE1 0AA, UK)

This paper gives an overview of research into classroom acoustics, highlighting the importance of a good acoustic environment in schools to enhance teaching and learning. The paper is aimed at a general audience of people interested in education and school design. In the past 30 years there has been a great deal of research into the effects of noise and poor acoustics in schools on children and teachers. It has been shown in many studies that children have difficulty hearing and understanding their teachers in noise, and both external environmental noise and noise within a school affect children's academic performance. Furthermore many teachers suffer from voice and throat problems which may be attributable to a poor acoustic environment in the classroom. The acoustic design of a classroom has a direct influence upon noise levels and the intelligibility of speech. Poor sound insulation and excessive reverberation have the potential to increase noise levels and reduce speech intelligibility. However, despite the introduction in many countries of legislation or guidelines for acoustic design of schools, in general acoustics still has a low priority in school design and many schools, old and new, fail to meet the current standards. 